\title{
BERNSTEIN-GELFAND-GELFAND SEQUENCES
}

\author{
ANDREAS ČAP, JAN SLOVÁK, AND VLADIMÍR SOUČEK
}

\begin{abstract}
This paper is devoted to the study of geometric structures modeled on homogeneous spaces $G / P$, where $G$ is a real or complex semisimple Lie group and $P \subset G$ is a parabolic subgroup. We use methods from differential geometry and very elementary finite-dimensional representation theory to construct sequences of invariant differential operators for such geometries, both in the smooth and the holomorphic category. For $G$ simple, these sequences specialize on the homogeneous model $G / P$ to the celebrated (generalized) Bernstein-Gelfand-Gelfand resolutions in the holomorphic category, while in the smooth category we get smooth analogs of these resolutions. In the case of geometries locally isomorphic to the homogeneous model, we still get resolutions, whose cohomology is explicitly related to a twisted de Rham cohomology. In the general (curved) case we get distinguished curved analogs of all the invariant differential operators occurring in Bernstein-GelfandGelfand resolutions (and their smooth analogs).

On the way to these results, a significant part of the general theory of geometrical structures of the type described above is presented here for the first time.
\end{abstract}

\section{INTRODUCTION}

Our approach to geometries modeled on homogeneous spaces goes back to E. Cartan's notion of an 'espace generalisé'. The central objects for such geometries are suitably normalized Cartan connections in the sense commonly adopted, see e.g. [31]. The models for the geometries considered in this paper are homogeneous spaces of the type $G / P$, where $G$ is real or complex semisimple and $P \subset G$ is a parabolic subgroup. In this case, there is a close link to the project of parabolic invariant theory suggested by Ch. Fefferman in 17] and in view of this context we talk about the (real and complex) parabolic geometries.

We explore the semi-holonomic jet modules and we use implicitly the cohomological information given by Kostant's version of the Bott-BorelWeil theorem in order to construct sequences of homomorphisms between jet-modules, which in turn give rise to sequences of invariant differential operators expressed in terms of the invariant derivatives with respect to Cartan connections, on all (curved) geometries in question. These sequences are differential complexes if certain twisted de Rham sequences are complexes, and then they compute the same cohomology. In particular, this always happens for the homogeneous models themselves and then our sequences specialize to the Bernstein-Gelfand-Gelfand resolutions well known from representation theory for complex $G / P$, while their real smooth analogues are provided for all real forms of this situation. 
In spite of the fact that we have mentioned a few concepts from representation theory, we want to underline that no deeper aspects of representation theory are used in the construction of our new sequences of invariant operators and in the discussion of their basic properties. In particular, no infinite dimensional representation theory is needed. It is rather the language and the way of thinking of representation theory that is essential (in a similar way as the categorical language is useful in mathematics even if no deep results of category theory are used). In order to stress this feature, we have postponed the more detailed analysis of the structure of the sequences to a forthcoming second part of the article and we hope that the first part is accessible for differential geometers without a deeper background in representation theory. We also provide a quite detailed exposition of the necessary algebraic background. In particular we have included two appendices covering some material which is rather well known in representation theory.

The first general geometric theory close to our needs had been worked out in the series of papers by N. Tanaka and his school aiming at the original equivalence problem of E. Cartan, see [34, 35, 27] and the references therein. Our inspiration comes, however, rather from the interest in the links between twistor theory and representation theory, as explained in the book [2]. In the generality we need, the normalized Cartan connections were constructed in [7] first. We have been also influenced by the translation principle in representation theory (see [4, 5] for example) and, in particular, by some ideas in the second part of Baston's paper [1]. Some arguments and proofs in the latter paper seem very unclear to us, however.

There are also many treatments of specific examples of parabolic geometries in the literature, including e.g. projective, conformal, almost Grassmannian, and CR-geometries. Most of these well known geometries correspond to the so called $|1|$-graded Lie algebras $\mathfrak{g}$ which can be equivalently expressed by the requirement that the tangent spaces correspond to irreducible representations of the parabolic subgroup $P$. Our theory of semi-holonomic jet-modules is in fact a generalization of the approach worked out for all real $|1|$-graded algebras in our former papers [8, 9, 10] (and this paper could be also viewed as a fourth part of this series expanded to the full generality of parabolic geometries). On the other hand, there are only few explicit examples of curved analogues of the Bernstein-Gelfand-Gelfand resolutions available in the literature, see e.g. [14], and in fact only the case of conformal Riemannian geometries has been studied systematically, see [19] and [16] for two different approaches. For an introduction addressed to wide audience, see the forthcoming paper 13.

Let us indicate the structure of the paper. In the next section, we first collect the necessary information on $|k|$-graded Lie algebras and the structure of the corresponding Lie groups, and then real and complex parabolic geometries are introduced (cf. 2.7). Our point of view is that the geometry on a manifold $M$ is given by a choice of a Cartan connection (with possible further normalization) and we are interested in the general calculus which such a choice offers. In a certain sense, this is similar to the rôle of the general calculus for linear connections in Riemannian geometry by application to the Levi-Civita connection. Thus we only briefly discuss the more classical underlying geometrical information on the manifolds $M$ themselves and 
the question of constructing a (normalized) Cartan connection from these more basic data, cf. 2.10. See [0, 27] for more information on this aspect. We also introduce the concepts of natural bundles and operators for parabolic geometries in the end of Section 2 .

The third section deals with our basic algebraic tool, the semi-holonomic jet modules. The invariant derivative with respect to Cartan connections then leads to the notion of strongly invariant differential operators which are defined by means of $P$-module homomorphisms. As a first application, we introduce the twisted exterior derivatives which are certain torsion adjusted versions of the covariant exterior derivatives induced by the Cartan connections on certain bundles.

The main results are stated and proved in Section 4. Referring implicitly to the structure of the Lie algebra cohomologies, we first embed the natural vector bundles corresponding to cohomologies into exterior forms by means of distinguished differential operators $L$, see Theorem 1.8. Then we use the twisted exterior derivatives in order to construct explicitly many $P-$ module homomorphisms of the semi-holonomic jet modules, cf. Proposition 4.9. The corresponding invariant differential operators build the BernsteinGelfand-Gelfand sequences. Finally we discuss the conditions under which these sequences form differential complexes, and we discuss their cohomologies, cf. 4.134 .15 .

Finally, we illustrate briefly the achievements on at least one non-trivial parabolic geometry and this is done in Section 5 .

Throughout the paper, we discuss the real and complex manifolds and groups at the same time. We should point out however, that the relation between the real and complex settings deserves more attention. In fact, we are able to present both smooth and holomorphic results in one line of arguments, because our point is to use the $P$-module homomorphisms in order to construct the sequences of operators. The distinction is hidden in the explicit structure of the Lie algebra cohomologies, which we use only implicitly. One should say, however, this does not mean that working out the details for one real form gives explicit results for all other real or complex forms of the group in question. This ambiguity disappears only if we restrict ourselves to complex representations of the real forms.

A more detailed discussion of our Bernstein-Gelfand-Gelfand sequences requires a deeper study of the cohomological information. Essentially, the non-trivial operators between the irreducible bundles in the sequence correspond to arrows in the Hasse diagram of the parabolic subalgebras and the knowledge of this structure leads to quite explicit information on the individual operators. We have preferred to postpone all considerations which need more involved information from representation theory to a prospective continuation in order to keep the flavor of this article.

Acknowledgements. The research evolved during a stay of the first two authors at the University of Adelaide supported by the Australian Research Council, and during the meetings of all three authors at the Erwin Schrödinger Institute for Mathematical Physics in Vienna, the Masaryk University in Brno, and the Charles University in Prague. The institutional 
support by GAČR, Grant Nr. 201/99/0675 has been essential, too. Our particular thanks are due to Michael Eastwood who explained to us several aspects of the Bernstein-Gelfand-Gelfand resolutions.

\section{Parabolic Geometries}

In this section we review basic facts about $|k|$-graded Lie algebras and we give basic definitions on parabolic geometries and invariant differential operators on manifolds equipped with geometries of that type. Most of the facts on the algebras go back to [34, 35], see also [0] which is fully compatible in notation.

2.1. Definition. Let $\mathbb{K}$ be $\mathbb{R}$ or $\mathbb{C}$. A $|k|$-graded Lie algebra over $\mathbb{K}, k \in \mathbb{N}$ is a Lie algebra $\mathfrak{g}$ over $\mathbb{K}$ together with a decomposition

$$
\mathfrak{g}=\mathfrak{g}_{-k} \oplus \cdots \oplus \mathfrak{g}_{-1} \oplus \mathfrak{g}_{0} \oplus \mathfrak{g}_{1} \oplus \cdots \oplus \mathfrak{g}_{k}
$$

such that $\left[\mathfrak{g}_{i}, \mathfrak{g}_{j}\right] \subset \mathfrak{g}_{i+j}$ and such that the subalgebra $\mathfrak{g}_{-}:=\mathfrak{g}_{-k} \oplus \cdots \oplus \mathfrak{g}_{-1}$ is generated by $\mathfrak{g}_{-1}$. In the whole paper, we will only deal with semisimple $|k|$-graded Lie algebras.

By $\mathfrak{p}$ we will denote the subalgebra $\mathfrak{g}_{0} \oplus \cdots \oplus \mathfrak{g}_{k}$ of $\mathfrak{g}$, and by $\mathfrak{p}_{+}$the subalgebra $\mathfrak{g}_{1} \oplus \cdots \oplus \mathfrak{g}_{k}$ of $\mathfrak{p}$.

There is always a unique element $E \in \mathfrak{g}$ whose adjoint action is given by $[E, X]=\ell X$ for $X \in \mathfrak{g}_{\ell}$. The element $E$ is contained in the center of the subalgebra $\mathfrak{g}_{0}$, which is always reductive. Using this, one shows that any ideal of $\mathfrak{g}$ is homogeneous. Thus, a semisimple $|k|$-graded Lie algebra is always a direct sum of simple $\left|k_{i}\right|$-graded Lie algebras, where all $k_{i} \leq k$. Hence, one usually can reduce most discussions to the simple case. When dealing with the semisimple case, we have to assume that none of the simple factors is contained in $\mathfrak{g}_{0}$, for technical reasons. Since basically we are interested in homogeneous spaces $G / P$, where $G$ is a Lie group with Lie algebra $\mathfrak{g}$ and $P$ an appropriate subgroup with Lie algebra $\mathfrak{p}$, and their curved analogs, this is not really a restriction.

For each $i=1, \ldots, k$, the Killing form of $\mathfrak{g}$ induces an isomorphism $\mathfrak{g}_{i} \cong$ $\mathfrak{g}_{-i}^{*}$ of $\mathfrak{g}_{0}$-modules. Finally, the powers of $\mathfrak{p}_{+}$are given by $\mathfrak{p}_{+}^{i}=\mathfrak{g}_{i} \oplus \cdots \oplus \mathfrak{g}_{k}$, for $i=1, \ldots, k$. See e.g. [35, Section 3] for details.

2.2. In the complex case, the meaning of a $|k|$-grading is particularly simple to describe. One can show that there always exists a Cartan subalgebra $\mathfrak{h} \subset \mathfrak{g}$ which contains the element $E$ from above, and a choice of positive roots $\Delta_{+}$for $\mathfrak{h}$ such that all root spaces corresponding to simple roots are either contained in $\mathfrak{g}_{0}$ or in $\mathfrak{g}_{1}$. Denoting by $\Sigma$ the set of those simple roots, whose root spaces are contained in $\mathfrak{g}_{1}$, one sees that the grading on $\mathfrak{g}$ is given by the $\Sigma$-height of roots. That is, if $\alpha$ is a root, then the root space $\mathfrak{g}_{\alpha}$ is contained in $\mathfrak{g}_{i}$, where $i$ is the sum of all coefficients of elements of $\Sigma$ in the expansion of $\alpha$ as a linear combination of simple roots. In particular, this implies that the subalgebra $\mathfrak{p}$ is always a parabolic subalgebra of $\mathfrak{g}$, and $\mathfrak{p}=\mathfrak{g}_{0} \oplus \mathfrak{p}_{+}$is exactly the Levi decomposition of $\mathfrak{p}$ into a reductive and a nilpotent part.

Conversely, if $\mathfrak{g}$ is complex and semisimple and $\mathfrak{p} \subset \mathfrak{g}$ is a parabolic subalgebra, then one can find a Cartan subalgebra and a set of positive roots such that $\mathfrak{p}$ is the standard parabolic corresponding to a set $\Sigma$ of 
simple roots. But then the $\Sigma$-height as defined above gives a $|k|$-grading on $\mathfrak{g}$, where $k$ is the $\Sigma$-height of the maximal root of $\mathfrak{g}$, such that $\mathfrak{p}=\mathfrak{g}_{0} \oplus \cdots \oplus \mathfrak{g}_{k}$. See e.g. [22, p. 88] or [2, Section 2] for more details.

Thus, in the complex case giving a $|k|$-grading on $\mathfrak{g}$ is the same thing as giving a parabolic subalgebra $\mathfrak{p}$ of $\mathfrak{g}$. Therefore, complex $|k|$-graded semisimple Lie algebras can be conveniently denoted by Dynkin diagrams with crossed nodes. That is, given a $|k|$-graded semisimple complex Lie algebra we may assume that $\mathfrak{p}$ is the standard parabolic subalgebra corresponding to a set $\Sigma$ of simple roots. Then we denote the $|k|$-graded Lie algebra $\mathfrak{g}$ by crossing out the nodes corresponding to the simple roots contained in $\Sigma$ in the Dynkin diagram of $\mathfrak{g}$. See the book [2] for a detailed discussion of the Dynkin diagram notation for parabolic subalgebras.

Finally note that for a $|k|$-graded Lie algebra $\mathfrak{g}$ over $\mathbb{R}$ the complexification $\mathfrak{g}^{\mathbb{C}}$ of $\mathfrak{g}$ is $|k|$-graded, too. So in general we deal with certain real forms of pairs $(\mathfrak{g}, \mathfrak{p})$, where $\mathfrak{g}$ is complex and semisimple and $\mathfrak{p}$ is a parabolic in $\mathfrak{g}$. The classification of all these real forms is provided in [35, Section 4].

2.3. Suppose that $\mathfrak{g}$ is $|k|$-graded and semisimple over $\mathbb{K}=\mathbb{R}$ or $\mathbb{C}$, and let $G$ be any Lie group with Lie algebra $\mathfrak{g}$. (We do not assume that $G$ is connected.) Then we can define subgroups $G_{0} \subset P \subset G$ as follows: $G_{0}$ consists of all elements of $G$ such that the adjoint action $\operatorname{Ad}(g): \mathfrak{g} \rightarrow \mathfrak{g}$ of $g$ preserves the grading of $\mathfrak{g}$. By $P$ we denote the subgroup of all elements $g \in G$ such that $\operatorname{Ad}(g)$ preserves the filtration by right ends induced by the grading of $\mathfrak{g}$, i.e. $\operatorname{Ad}(g)\left(\mathfrak{g}_{i}\right) \subset \mathfrak{g}_{i} \oplus \cdots \oplus \mathfrak{g}_{k}$. By definition $G_{0}$ is a subgroup of $P$, and one easily verifies that $G_{0}$ and $P$ have Lie algebras $\mathfrak{g}_{0}$ and $\mathfrak{p}$, respectively, see e.g. [7, 2.9]. Moreover, it can be shown that if $\mathfrak{g}$ is simple, then $P$ equals the normalizer $N_{G}(\mathfrak{p})$ of $\mathfrak{p}$ in $G$, so it is the usual parabolic subgroup associated to the parabolic subalgebra $\mathfrak{p}$.

The following proposition clarifies the structure of the group $P$ :

Proposition. Let $g \in P$ be any element. Then there exist unique elements $g_{0} \in G_{0}$ and $X_{i} \in \mathfrak{g}_{i}$ for $i=1, \ldots, k$, such that

$$
g=g_{0} \exp \left(X_{1}\right) \ldots \exp \left(X_{k}\right) \text {. }
$$

Proof. See [7, 2.10].

2.4. For $i=1, \ldots, k$ we define a subgroup $P_{+}^{i} \subset P$ as the image under the exponential map of $\mathfrak{g}_{i} \oplus \cdots \oplus \mathfrak{g}_{k}$, and we write $P_{+}$for $P_{+}^{1}$. Then we have $P \supset P_{+} \supset P_{+}^{2} \supset \cdots \supset P_{+}^{k}$. The subgroup $P_{+} \subset P$ is obviously normal and by Proposition 2.3 we have $P / P_{+} \cong G_{0}$, so $P$ is the semidirect product of $G_{0}$ and the normal nilpotent subgroup $P_{+}$. More generally, for each $i>1$ we see that $P / P_{+}^{i}$ is the semidirect product of $G_{0}$ and the normal nilpotent subgroup $P_{+} / P_{+}^{i}$.

The adjoint action of $P$ on $\mathfrak{g}$ by definition preserves any of the subspace $\mathfrak{g}_{i} \oplus \cdots \oplus \mathfrak{g}_{k}$ for $i=-k, \ldots, k$. Thus for each $i=-k, \ldots, k$ and $j>i$ we get an induced action of $P$ on the quotient $\mathfrak{g}_{i} \oplus \cdots \oplus \mathfrak{g}_{k} /\left(\mathfrak{g}_{j} \oplus \cdots \oplus \mathfrak{g}_{k}\right)$. With a slight abuse of notation, we will denote this $P$-module by $\mathfrak{g}_{i} \oplus \cdots \oplus \mathfrak{g}_{j-1}$. Again by Proposition 2.3, the action of $P_{+}^{j-i}$ on $\mathfrak{g}_{i} \oplus \cdots \oplus \mathfrak{g}_{j-1}$ is trivial, so the action of $P$ on this space is induced by an action of $P / P_{+}^{j-i}$. In particular, we get an action of $P$ on $\mathfrak{g}_{-}=\mathfrak{g} / \mathfrak{p}$, which is induced by an action of $P / P_{+}^{k}$. 
There is another important consequence of Proposition 2.3: Suppose that $\mathbb{V}$ and $\mathbb{W}$ are $P$-modules and that $\Phi: \mathbb{V} \rightarrow \mathbb{W}$ is a linear mapping. Suppose that $\Phi$ is equivariant for the action of $G_{0}$ and for the (infinitesimal) action of $\mathfrak{g}_{1}$. Since $\mathfrak{p}_{+}$is generated by $\mathfrak{g}_{1}$ this implies equivariancy with respect to $\mathfrak{p}_{+}$and thus also with respect to $P_{+}$, so using Proposition 2.3 we see that $\Phi$ is actually a homomorphism of $P$-modules. This will be technically very important in the sequel.

2.5. For a Lie group $G$ with $|k|$-graded semisimple Lie algebra $\mathfrak{g}$ and the subgroup $P$ defined in 2.3 above, consider the homogeneous space $G / P$. This homogeneous space is the flat model for the parabolic geometry of the type $(G, P)$ that we are going to study. It is well known that the canonical projection $G \rightarrow G / P$ is a principal fiber bundle with structure group $P$.

If $G$ is a complex Lie group, then $P$ is a parabolic subgroup, so $G / P$ is a generalized flag manifold, and thus in particular a compact complex manifold. In the real case, $G / P$ need not be compact in general, as the example of the conformal spheres in indefinite signature shows.

Next suppose that $\lambda: P \rightarrow \mathrm{GL}(\mathbb{V})$ is a representation of $P$ on a finite dimensional vector space $\mathbb{V}$. Then we can form the associated bundle $V:=G \times{ }_{P} \mathbb{V} \rightarrow G / P$. This is a homogeneous vector bundle, that is the canonical left action of $G$ on $G / P$ lifts to a left action of $G$ on $V$ by vector bundle homomorphisms. Conversely, given a homogeneous vector bundle $E \rightarrow G / P$, consider the fiber $\mathbb{E}$ of $E$ over the canonical base point $o \in G / P$. Since the action of any element of $P$ on $G / P$ maps $o$ to itself, the action of $G$ on $E$ induces a representation of $P$ on $\mathbb{E}$ and one easily verifies that $G \times_{P} \mathbb{E}$ and $E$ are isomorphic homogeneous vector bundles (i.e. there is a $G$-equivariant isomorphism of vector bundles between them). Consequently, there is a bijective correspondence between finite dimensional representations of $P$ and homogeneous vector bundles over $G / P$. In the case where $G$ is a complex Lie group, the action of $G$ on $G / P$ is holomorphic and there is a bijective correspondence between holomorphic finite dimensional representations of $P$ and holomorphic homogeneous vector bundles over $G / P$ (that is holomorphic bundles with holomorphic $G$-actions).

In particular, the tangent and cotangent bundles of $G / P$ are homogeneous vector bundles. One easily verifies that they correspond to the representations of $P$ on $\mathfrak{g}_{-} \cong \mathfrak{g} / \mathfrak{p}$ and $\mathfrak{p}_{+}$induced by the adjoint action, respectively. In the complex case, these representations induce the holomorphic tangent and cotangent bundle.

For a homogeneous vector bundle $E \rightarrow G / P$ consider the space $\Gamma(E)$ of smooth sections of $E$. There is an induced action of $G$ on this space given by $(g \cdot s)(x)=g \cdot\left(s\left(g^{-1} \cdot x\right)\right)$ for $x \in G / P$. In the complex case, we can deal similarly with the spaces of holomorphic sections.

Definition. Let $E$ and $F$ be homogeneous vector bundles over $G / P$. A (linear) invariant differential operator $D: \Gamma(E) \rightarrow \Gamma(F)$ is a linear differential operator $D$ which is equivariant for the $G$-actions constructed above.

2.6. If $D$ is of order $\leq r$, then it is induced by a vector bundle homomorphism $\tilde{D}: J^{r}(E) \rightarrow F$, where $J^{r}(E)$ is the $r$-th jet prolongation of $E$. Now simply by functoriality of the $r$-th jet prolongation, $J^{r}(E)$ is again a 
homogeneous vector bundle, and the invariance of $D$ is equivalent to the fact that $\tilde{D}$ is equivariant for the $G$-actions on $J^{r}(E)$ and $F$. Since $G$ acts transitively on $G / P$, the homomorphism $\tilde{D}$ is actually determined by its restriction $\tilde{D}: J^{r}(E)_{o} \rightarrow F_{o}$ to the fiber over $o \in G / P$, and by invariance of $D$, this map is $P$-equivariant.

Conversely, a $P$-homomorphism $J^{r}(E)_{o} \rightarrow F_{o}$ extends uniquely to a $G^{-}$ homomorphism $J^{r}(E) \rightarrow F$ and thus gives rise to an invariant differential operator. Thus, invariant differential operators $\Gamma(E) \rightarrow \Gamma(F)$ of order $\leq r$ are in bijective correspondence with $P$-homomorphisms $J^{r}(E)_{o} \rightarrow F_{o}$. To avoid the restriction on the order, one can simply pass to infinite jets and we obtain that invariant differential operators $\Gamma(E) \rightarrow \Gamma(F)$ are in bijective correspondence with $P$-homomorphisms $J^{\infty}(E)_{o} \rightarrow F_{o}$, which factorize over some $J^{r}(E)$.

Surprisingly, the problem of determining all such homomorphisms has a nice reformulation in term of (infinite-dimensional) representation theory, which has led to a complete solution in several cases. Namely, suppose that $E$ and $F$ correspond to representations $\mathbb{E}$ and $\mathbb{F}$ of $P$, respectively. For the dual representation $\mathbb{E}^{*}$, one can form the induced module $\mathcal{U}(\mathfrak{g}) \otimes_{\mathcal{U}(\mathfrak{p})} \mathbb{E}^{*}$, which is a $(\mathfrak{g}, P)$-module, i.e. it admits compatible actions of $\mathfrak{g}$ and $P$. In the case where $\mathfrak{p} \subset \mathfrak{g}$ is the Borel subalgebra (i.e. the minimal parabolic) and $\mathbb{E}$ is irreducible, these are the Verma-modules while for general $\mathfrak{p}$ and irreducible $\mathbb{E}$, they are called generalized Verma-modules. By a dualization argument and Frobenius reciprocity one shows that for $\mathbb{E}$ and $\mathbb{F}$ irreducible, the space of all $P$-module homomorphisms $J^{\infty}(E)_{o} \rightarrow F_{o}$, which factorize over some $J^{r}(E)_{o}$ is isomorphic to the space of all $(\mathfrak{g}, P)$-homomorphisms $\mathcal{U}(\mathfrak{g}) \otimes_{\mathcal{U}(\mathfrak{p})} \mathbb{F}^{*} \rightarrow \mathcal{U}(\mathfrak{g}) \otimes_{\mathcal{U}(\mathfrak{p})} \mathbb{E}^{*}$. Since these considerations are essential for understanding of the links of our development to the standard BernsteinGelfand-Gelfand resolutions, we provide some more details in Appendix Appendix A.

Let us remark however that while there is a complete classification of homomorphisms of Verma-modules in the complex case in [3], the classification of homomorphisms of generalized Verma modules is a very difficult problem, which is unsolved in general (even in the complex case). There is a complete classification in the case of real rank one for one dimensional representations in [26] and for general representations in [4] and [5]. The problem in the case of generalized Verma modules is the following: One has a class of homomorphisms which are induced by homomorphisms of Verma modules, the so-called standard homomorphisms. These are exactly the homomorphisms which occur in Bernstein-Gelfand-Gelfand resolutions. But it may happen that a homomorphism of Verma modules induces the zero-homomorphism between generalized Verma modules, and in this situation there may still be nonzero homomorphisms (the so called non-standard homomorphisms).

2.7. Parabolic geometries. Some geometries can be viewed as curved analogs of the homogeneous spaces $G / P$ considered above. For the purpose of this paper, the best way to define them is simply as generalized spaces in the sense of E. Cartan.

Let $\mathfrak{g}=\mathfrak{g}_{-k} \oplus \cdots \oplus \mathfrak{g}_{k}$ be a real $|k|$-graded Lie algebra and let $G$ be a Lie group with Lie algebra $\mathfrak{g}$. Let $G_{0}$ and $P$ be the subgroups of $G$ defined 
in 2.3 above. Then we define a (real) parabolic geometry of type $(G, P)$ on a smooth manifold $M$ to be a principal $P$-bundle $\mathcal{G} \rightarrow M$ equipped with a Cartan connection of type $(G, P)$, i.e. a differential form $\omega \in \Omega^{1}(\mathcal{G}, \mathfrak{g})$ such that

(1) $\omega\left(\zeta_{X}\right)=X$ for all $X \in \mathfrak{p}$

(2) $\left(r^{b}\right)^{*} \omega=\operatorname{Ad}\left(b^{-1}\right) \circ \omega$ for all $b \in P$

(3) $\left.\omega\right|_{T_{u} \mathcal{G}}: T_{u} \mathcal{G} \rightarrow \mathfrak{g}$ is a linear isomorphism for all $u \in \mathcal{G}$.

Here $\zeta_{X}$ denotes the fundamental vector field generated by $X \in \mathfrak{p}$ and $r^{b}$ denotes the principal right action of $b \in P$. Thus, $\omega$ gives a smooth $P-$ equivariant trivialization of the tangent bundle of $\mathcal{G}$, which reproduces the generators of fundamental fields. Each $X \in \mathfrak{g}$ defines the constant vector field $\omega^{-1}(X)$ given by $\omega^{-1}(X)(u)=\omega_{u}^{-1}(X) \in T_{u} \mathcal{G}$. Clearly, a parabolic geometry of type $(G, P)$ on $M$ can only exist if $M$ has the same dimension as $G / P$.

In the complex setting, the Lie algebras and groups, as well as the manifold $M$ are complex and the above definition remains unchanged except for the replacement of smooth by holomorphic. Thus a complex parabolic geometry of the type $(G, P)$ on a complex manifold $M$ is given by a holomorphic principal fiber bundle equipped with a holomorphic absolute parallelism $\omega$ with the three properties listed above.

The (real or complex) homogeneous space $G / P$ always carries a canonical parabolic geometry, namely $\mathcal{G}=G$ and the Cartan connection is given by the left Maurer Cartan form. Then the constant vector fields are exactly the left invariant fields on $G$.

It is fairly easy to make the parabolic geometries as defined above into a category. Let $(\mathcal{G}, \omega)$ be a real parabolic geometry on $M$ and $\left(\mathcal{G}^{\prime}, \omega^{\prime}\right)$ be a parabolic geometry on $M^{\prime}$, and suppose that $\Phi: \mathcal{G} \rightarrow \mathcal{G}^{\prime}$ is a smooth homomorphism of principal $P$-bundles, such that the induced map $\underline{\Phi}: M \rightarrow M^{\prime}$ is a local diffeomorphism. Then for any point $u \in \mathcal{G}$ the tangent map $T_{u} \Phi: T_{u} \mathcal{G} \rightarrow T_{\Phi(u)} \mathcal{G}^{\prime}$ is a linear isomorphism, and using this, one immediately verifies that $\Phi^{*} \omega^{\prime}:=\omega^{\prime} \circ T \Phi$ is a Cartan connection on $\mathcal{G}$. Now we define a morphism from $(\mathcal{G}, \omega)$ to $\left(\mathcal{G}^{\prime}, \omega^{\prime}\right)$ to be a homomorphism $\Phi$ of principal bundles such that the induced map $\underline{\Phi}: M \rightarrow M^{\prime}$ is a local diffeomorphism and such that $\omega=\Phi^{*} \omega^{\prime}$. For complex parabolic geometries we additionally require all maps to be holomorphic.

Note that any homomorphism $\Phi: \mathcal{G} \rightarrow \mathcal{G}^{\prime}$ of principal bundles which lies over a local diffeomorphism can be viewed as a morphism $\left(\mathcal{G}, \Phi^{*} \omega^{\prime}\right) \rightarrow$ $\left(\mathcal{G}^{\prime}, \omega^{\prime}\right)$. More generally, if $\left(\mathcal{G}^{\prime}, \omega^{\prime}\right)$ is a parabolic geometry on $M^{\prime}$ and $f$ : $M \rightarrow M^{\prime}$ is a local diffeomorphism, then we can form the pullback bundle $f^{*} \mathcal{G}^{\prime} \rightarrow M$. Then there is an induced homomorphism $\Phi: f^{*} \mathcal{G}^{\prime} \rightarrow \mathcal{G}^{\prime}$ of principal bundles which lies over $f$, and we get an induced morphism $\left(f^{*} \mathcal{G}^{\prime}, \Phi^{*} \omega^{\prime}\right) \rightarrow\left(\mathcal{G}^{\prime}, \omega^{\prime}\right)$

2.8. For some purposes, the category of parabolic geometries as defined above is too large, and one has to impose certain restrictions. Usually, these restrictions are on the curvature of the Cartan connection. Initially, the curvature of a Cartan connection $\omega$ is defined as the $\mathfrak{g}$-valued two-form 
$K \in \Omega^{2}(\mathcal{G}, \mathfrak{g})$ defined by the structure equation

$$
K(\xi, \eta)=d \omega(\xi, \eta)+[\omega(\xi), \omega(\eta)],
$$

where $\xi$ and $\eta$ are vector fields on $\mathcal{G}$ and the bracket is in $\mathfrak{g}$. Using the properties of $\omega$ one immediately verifies that $K$ is horizontal and equivariant. In particular, this implies that $K$ is uniquely determined by the curvaturefunction $\kappa: \mathcal{G} \rightarrow \Lambda^{2} \mathfrak{g}_{-}^{*} \otimes \mathfrak{g}$ defined by $\kappa(u)(X, Y)=K(u)\left(\omega_{u}^{-1}(X), \omega_{u}^{-1}(Y)\right)$. There are two natural ways to split $\kappa$ into components. First, the splitting of $\mathfrak{g}$ induces a splitting of $\kappa$ according to the values in $\mathfrak{g}$. In particular, we can split $\kappa=\kappa_{-} \oplus \kappa_{\mathfrak{p}}$ according to the splitting $\mathfrak{g}=\mathfrak{g}_{-} \oplus \mathfrak{p}$. Following the classical terminology for affine connections, $\kappa_{-}$is called the torsion of $\omega$. The other possibility is to split $\kappa$ according to homogeneous components. We denote the homogeneous component of degree $i$ of $\kappa$ by $\kappa^{(i)}$. So $\kappa^{(i)}$ maps $\mathfrak{g}_{j} \otimes \mathfrak{g}_{k}$ to $\mathfrak{g}_{i+j+k}$.

Another important point is that the space $\Lambda^{2} \mathfrak{g}_{-}^{*} \otimes \mathfrak{g}$ is the second chain group $C^{2}\left(\mathfrak{g}_{-}, \mathfrak{g}\right)$ in the standard complex for the Lie algebra cohomology $H^{*}\left(\mathfrak{g}_{-}, \mathfrak{g}\right)$ of the nilpotent Lie algebra $\mathfrak{g}_{-}$with coefficients in the $\mathfrak{g}_{-}$-module

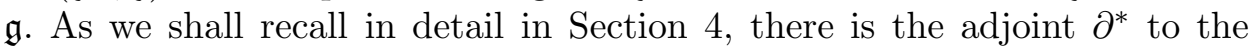
Lie algebra differential $\partial$ in this complex, so in particular, we have $\partial^{*}$ : $\Lambda^{2} \mathfrak{g}_{-}^{*} \otimes \mathfrak{g} \rightarrow \mathfrak{g}_{-}^{*} \otimes \mathfrak{g}$.

Definition. Let $(\mathcal{G}, \omega)$ be a (real or complex) parabolic geometry on a manifold $M$, and let $\kappa$ be the curvature of $\omega$. Then the parabolic geometry is called

(1) normal if $\partial^{*} \circ \kappa=0$.

(2) regular if it is normal and $\kappa^{(i)}=0$ for all $i \leq 0$.

(3) torsion-free if $\kappa_{-}=0$.

(4) flat if $\kappa=0$.

Note that forming the curvature of a Cartan connection is a natural operation. This means that if $\Phi: \mathcal{G} \rightarrow \mathcal{G}^{\prime}$ is a homomorphism of principal bundles and $\omega^{\prime}$ is a Cartan connection with curvature $K^{\prime}$ and curvature-function $\kappa^{\prime}$ then the curvature $K$ and curvature function $\kappa$ of the pullback $\Phi^{*} \omega^{\prime}$ are given by $K=\Phi^{*} K^{\prime}$ and $\kappa=\kappa^{\prime} \circ \Phi$, respectively. Since all the subclasses of parabolic geometries defined above are given by restricting the values of the curvature-function, morphisms into a parabolic geometry from one of the four subclasses can only come from geometries from the same subclass. Clearly, for any of the four subclasses the geometries belonging to the class form a full subcategory of the category of all parabolic geometries of fixed type.

2.9. Examples. Before we review the construction of parabolic geometries from underlying data, we present two well known examples.

Conformal structures. Consider $\mathbb{R}^{n}$ with coordinates $x_{1}, \ldots, x_{n}$ and the standard inner product $\langle$,$\rangle of signature (p, q)$, and $\mathbb{R}^{n+2}$ with coordinates $x_{0}, x_{1}, \ldots, x_{n}, x_{\infty}$ and the inner product associated to the quadratic form $2 x_{0} x_{\infty}+\left\langle\left(x_{1}, \ldots, x_{n}\right),\left(x_{1}, \ldots, x_{n}\right)\right\rangle$, which has signature $(p+1, q+1)$. Let $G=S O_{0}(p+1, q+1)$ be the connected component of the special orthogonal group of this metric. Then the Lie algebra $\mathfrak{g}$ of $G$ admits a $|1|$-grading by decomposing matrices into blocks of sizes 1, $n$, and 1, see e.g. [8, 3.3(2)]. 
The construction of the canonical Cartan connection for manifolds endowed with a conformal structure of signature $(p, q)$, originally due to E. Cartan (see [11]), shows that conformal structures of this signature are precisely the same thing as normal parabolic geometries corresponding to that choice of $G$ and $P$. See [8] for a construction of the canonical Cartan connection on conformal manifolds in a style similar to the approach of this paper. In this special situation, normal Cartan connections turn out to be automatically regular and torsion free, so three of the four subclasses defined in 2.8 above coincide. The flat parabolic geometries in this case are exactly the locally conformally flat manifolds.

Partially integrable almost CR-structures. The complex analog of the above construction leads to the partially integrable almost CR-structures which present another example of real parabolic geometries. Here we have to consider the complex vector space $\mathbb{C}^{n}$ with the standard Hermitian inner product of signature $(p, q)$ and $\mathbb{C}^{n+2}$ with the Hermitian inner product associated to $z_{0} \bar{z}_{\infty}+\bar{z}_{0} z_{\infty}+\left\langle\left(z_{1}, \ldots, z_{n}\right),\left(z_{1}, \ldots, z_{n}\right)\right\rangle$. Now we put $G=P S U(p+1, q+1)$ the quotient of the special unitary group corresponding to this Hermitian inner product by its center. Splitting the matrices in the Lie algebra $\mathfrak{g}$ of $G$ into blocks of sizes $1, n$, and 1 this time gives rise to a $|2|$-grading. The construction of canonical Cartan connections in [7] shows that partially integrable almost CR-structures with non-degenerate Levi-form of signature $(p, q)$ are exactly the same thing as regular parabolic geometries corresponding to $G$ (see [7, 4.14]). In this case, three of the four subclasses of geometries defined in 2.8 above are really different: The torsion free parabolic geometries in this case are precisely the $\mathrm{CR}$-structures (see [7, 4.16]), and the flat ones are those which are locally isomorphic to the homogeneous model. The only coincidence in this case is that normal parabolic geometries are automatically regular.

2.10. Underlying structures. These two examples already show that identifying a geometrical structure on a manifold as a parabolic geometry should be rather the result of a theorem than a definition. In fact one can show in a fairly general setting that certain parabolic geometries are determined by underlying structures. This is the subject of the paper [7] which generalizes [34], see also [27] and [35]. To review the results, we first describe the underlying structures we have in mind.

Suppose that $(\mathcal{G}, \omega)$ is a regular parabolic geometry on a manifold $M$. The first thing we get out of this is a filtration $T M=T^{-k} M \supset T^{-k+1} M \supset \cdots \supset$ $T^{-1} M$ of the tangent bundle of $M$. This is given by defining $T^{i} M$ to be the set of those tangent vectors $\xi$ on $M$ for which there is a tangent vector $\tilde{\xi}$ in $T \mathcal{G}$ lying over $\xi$ with $\omega(\tilde{\xi}) \in \mathfrak{g}_{i} \oplus \cdots \oplus \mathfrak{g}_{k}$. The latter condition is independent of the choice of $\tilde{\xi}$ since changing the vector with fixed footpoint adds a vertical vector whose image under $\omega$ lies in $\mathfrak{p}$, while changing the footpoint leads to the adjoint action of an element of $P$, which by definition preserves the subspace $\mathfrak{g}_{i} \oplus \cdots \oplus \mathfrak{g}_{k}$. Clearly, this filtration has the property that the rank of $T^{i} M / T^{i+1} M$ equals the dimension of $\mathfrak{g}_{i}$ for all $i=-k, \ldots,-1$.

Now the underlying structures basically are given by considering the bundles $\mathcal{G} / P_{+}^{i} \rightarrow M$ for $i=1, \ldots, k$ and the "traces" of the Cartan connection 
that remain on these bundles. This "trace" on the bundle $\mathcal{G} / P_{+}^{i} \rightarrow M$ is a frame form of length $i$ in the sense of [7, 3.2]. For the case $i=1$ the geometric meaning of such a frame form is particularly easy to describe: It is exactly a reduction to the structure group $G_{0}$ of the associated graded vector bundle

$$
\operatorname{gr} T M=T^{-k} M / T^{-k+1} M \oplus \cdots \oplus T^{-2} M / T^{-1} M \oplus T^{-1} M
$$

to the tangent bundle $T M$. The fact that the curvature-function $\kappa$ of the regular Cartan connection $\omega$ has the property that $\kappa^{(i)}=0$ for all $i \leq 0$ is reflected in a property of the underlying frame forms called the structure equation, see [7, 3.4]. The bundle $\mathcal{G} / P_{+}^{i}$ together with the frame form of length $i$, which satisfies the structure equations is called the underlying $P$ frame bundle of degree $i$. Again, for $i=1$ this condition can be easily understood geometrically. It is equivalent to the fact that the algebraic Lie bracket on gr $T M$ which comes from the reduction to the group $G_{0}$ is induced by the Lie bracket of vector fields, that is it is given by a (generalized) Leviform.

Now the main result of [7] can be stated (with the help of the language of Dynkin diagrams for the pairs $(\mathfrak{g}, \mathfrak{p})$ mentioned in 2.2 above) as follows:

Let $(\mathfrak{g}, \mathfrak{p}), G, P$, and $G_{0}$ be as in 2.3 and suppose throughout that no simple factor of $\mathfrak{g}$ is contained in $\mathfrak{g}_{0}$ and $\mathfrak{g}$ does not contain a simple factor of type $A_{1}$. Then:

(1) If $(\mathfrak{g}, \mathfrak{p})$ does not contain any simple factor of one of the types

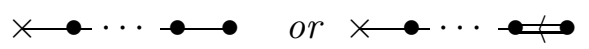

then any regular parabolic geometry can be reconstructed from the underlying $P$-frame bundle of degree one, and any $P$-frame bundle of degree one comes from a regular parabolic geometry. Thus, in all these cases regular parabolic geometries are the same thing as manifolds with filtered tangent bundle plus reductions of gr $T M$ to the group $G_{0}$ such that the resulting algebraic bracket is induced by the Lie bracket.

(2) If $\mathfrak{g}$ contains simple factors of one of the two above types, then any regular parabolic geometry can be reconstructed from the underlying $P$-frame bundle of degree two and any such bundle comes from a regular parabolic geometry. Moreover, any $P$-frame bundle of degree one can be extended (in various ways) to a $P$-frame bundle of degree two.

The classical examples of the second case are the projective structures where the $P$-frame bundle of degree one is simply the full frame bundle and all the structure is contained in the choice of an extension to a $P$-frame bundle of degree two. The other exceptional examples are the so called projective contact structures.

2.11. Natural bundles and operators. We will not go into much detail in the generalities about natural bundles and natural operators, but just outline the basic facts. We do not want to compare the various notions of naturality (this will be taken up elsewhere) but just show that the operators we are going to construct are natural (or invariant) in any reasonable sense.

Given a representation of $P$ on a vector space $\mathbb{V}$ and a parabolic geometry $(\mathcal{G} \rightarrow M, \omega)$ we can form the associated bundle $V M=\mathcal{G} \times{ }_{P} \mathbb{V} \rightarrow M$. If $\Phi: \mathcal{G} \rightarrow \mathcal{G}^{\prime}$ is a homomorphism of principal bundles which covers a local 
diffeomorphism $\underline{\Phi}: M \rightarrow M^{\prime}$, then we get an induced homomorphism of vector bundles $\overline{V M} \rightarrow V M^{\prime}$ which lies over the same map $\underline{\Phi}$ and restricts to a linear isomorphism in each fiber. To put it in another way, we get a functor from the category of parabolic geometries to the category of vector bundles over manifolds of the same dimension as $G / P$ and vector bundle homomorphisms which cover local diffeomorphisms and induce linear isomorphisms in each fiber such that the composition of the base functor with the given functor equals the base functor. Thus, we get a special case of a gauge natural bundle as defined in [24, Chapter XII].

Consider next a fixed category of real parabolic geometries, and two representations $\mathbb{V}$ and $\mathbb{W}$ of $P$. Let $V$ and $W$ be the corresponding natural vector bundles. A natural linear operator mapping sections of $V$ to sections of $W$ is defined to be a system of linear operators $D_{(\mathcal{G}, \omega)}: \Gamma(V M) \rightarrow \Gamma(W M)$, where $M$ is the base of $\mathcal{G}$ such that for any morphism $\Phi:(\mathcal{G}, \omega) \rightarrow\left(\mathcal{G}^{\prime}, \omega^{\prime}\right)$ we have

$$
\Phi^{*} \circ D_{\left(\mathcal{G}^{\prime}, \omega^{\prime}\right)}=D_{(\mathcal{G}, \omega)} \circ \Phi^{*} .
$$

This definition implies immediately, that each of the operators is local both in the section and in the Cartan connection: Suppose that $s \in \Gamma(V M)$ vanishes identically on an open subset $U \subset M$. Then there is an obvious inclusion morphism $i:\left(\left.\mathcal{G}\right|_{U},\left.\omega\right|_{U}\right) \rightarrow(\mathcal{G}, \omega)$ and $i^{*} s=0$. Thus also $i^{*}\left(D_{(\mathcal{G}, \omega)}(s)\right)=0$, i.e. $D_{(\mathcal{G}, \omega)}(s)$ is identically zero on $U$. Similarly, assume that $\omega$ and $\omega^{\prime}$ are two Cartan connections which coincide on $\left.\mathcal{G}\right|_{U}$. Then for any section $s \in \Gamma(V M)$ we have $\left.D_{(\mathcal{G}, \omega)}(s)\right|_{U}=\left.D_{\left(\mathcal{G}, \omega^{\prime}\right)}(s)\right|_{U}$. In particular, the classical Peetre theorem implies that each of the operators $D_{(\mathcal{G}, \omega)}$ is locally over $M$ a finite order differential operator with respect to the arguments in the vector bundles and the Cartan connection.

For complex parabolic geometries, we deal with holomorphic representations of $P$, the natural vector bundles are holomorphic, and the natural operators act on holomorphic sections. Let us also remark that all these concepts extend to non-linear objects without essential changes.

2.12. The natural operators on the category of flat parabolic geometries are particularly easy to describe: It is a classical result on Cartan connections that any flat parabolic geometry is locally isomorphic to the homogeneous model $G / P$ (see [7, 4.12] for a proof in the setting of parabolic geometries). This immediately implies that any natural operator on the category of flat parabolic geometries is uniquely determined by its value on the homogeneous model $G / P$, i.e. the parabolic geometry $(G \rightarrow G / P, \omega)$. Moreover, an operator on the flat model extends to a natural operator on the category of flat parabolic geometries if and only if it is natural with respect to all automorphisms of $(G, \omega)$. The left multiplication by any element of $G$ induces an automorphism of the principal bundle $G \rightarrow G / P$ and by left invariance of the Maurer Cartan form this actually is an automorphism of the parabolic geometry $(G, \omega)$. On the other hand, by [31, Theorem 3.5.2] the only smooth functions $G \rightarrow G$ which pull back the Maurer Cartan form to itself are the constant left translations. Thus $G$ is exactly the group of all automorphisms of $(G, \omega)$. But this immediately implies that an operator on the homogeneous model extends to a natural operator on the category of flat parabolic geometries if and only if it is invariant in the sense of definition 2.5. Thus for 
the flat case, the description of natural operators is equivalent to a problem in representation theory.

Usually, the question on more general natural operators is then posed (in the special cases that have been studied so far) as the question of the existence of curved analogs of invariant operators. This should be viewed as follows: As we discussed in 2.6, an invariant operator of order $r$ is induced by a $P$-module homomorphism $J^{r}(E)_{o} \rightarrow F_{o}$, which does not factor over $J^{r-1}(E)_{o}$. Now the kernel of the projection $J^{r}(E)_{o} \rightarrow J^{r-1}(E)_{o}$ is the bundle $S^{r} T^{*}(G / P) \otimes E$, so it corresponds to the representation $S^{r} \mathfrak{p}_{+} \otimes \mathbb{E}$. Thus the invariant operator gives rise to a $P$-module homomorphism $S^{r} \mathfrak{p}_{+} \otimes$ $\mathbb{E} \rightarrow \mathbb{F}$, which in turn gives a $G$-equivariant homomorphism between the corresponding homogeneous vector bundles which is precisely the symbol of the operator we started with. But this $P$-module homomorphism induces a homomorphism of associated bundles on any parabolic geometry, so for any parabolic geometry $(\mathcal{G}, \omega)$ over a manifold $M$, we get the corresponding homomorphism $S^{r} T^{*} M \otimes E M \rightarrow F M$. Now a curved analog of an invariant operator is a natural operator such that for each $(\mathcal{G}, \omega)$ the symbol of $D_{(\mathcal{G}, \omega)}$ is the above homomorphism. Otherwise put, the question is whether we can extend a given natural operator from the category of flat parabolic geometries to some larger category of parabolic geometries without changing its symbol, which, as a natural transformation, makes sense on any parabolic geometry.

2.13. We conclude this introductory section with some more remarks on the beautiful geometric structure underlying each parabolic geometry. This topic deserves much more attention than we could pay here and it will be studied in detail elsewhere. Some first steps have been done in 33.

Suppose that $(\mathcal{G}, \omega)$ is a real parabolic geometry on a manifold $M$. Then we have the tower of principal fiber bundles $\mathcal{G} \rightarrow \mathcal{G} / P_{+} \rightarrow M$ and the top level has the structure group $P_{+}$. Now using the Baker-Campbell-Hausdorff formula, Proposition 2.3 can be restated in the form that for any $g \in P$ there is a unique $g_{0} \in G_{0}$ and a unique $Z \in \mathfrak{p}_{+}$such that $g=g_{0} \exp (Z)$. But using this, one easily shows that the bundle $\mathcal{G} \rightarrow \mathcal{G} / P_{+}$admits global $G_{0}$-equivariant smooth sections. Namely, one can use a local trivialization of $\mathcal{G} \rightarrow M$ to construct equivariant sections over the preimage in $\mathcal{G} / P_{+}$of appropriate open subsets of $M$. Such local sections can then be glued to a global section using a partition of unity (compare with the proof of 8 , Lemma 3.6]). As in this last reference one also proves that the space of all these sections is an affine space modeled on the space $\Omega^{1}(M)$ of one-forms on $M$.

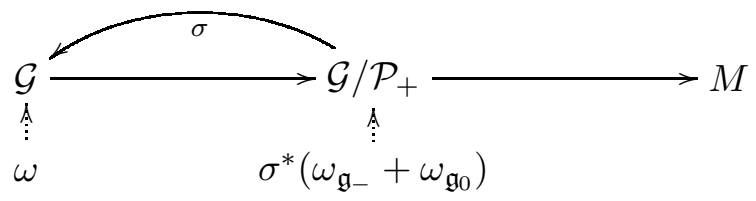

Each such global section $\sigma$ reduces the structure group of the tangent space $T M$ to $G_{0}$ and induces an affine connection $\gamma^{\sigma}=\sigma^{*}\left(\omega_{\mathfrak{g}_{-}}+\omega_{\mathfrak{g}_{0}}\right)$ on $T M$. This affine connection is $\sigma$-related to another Cartan connection $\omega^{\sigma}$ on $\mathcal{G}$, which differs from $\omega$ only in the $\mathfrak{p}_{+}-$component. The class of all connections $\gamma^{\sigma}$ is 
a straightforward generalization of Weyl structures on conformal geometries and all differential operators built of the Cartan connection $\omega$ can be expressed by uniform formulae in terms of these affine connections and their torsions and curvatures. The technique based on this general framework was developed systematically for all $|1|$-graded algebras $\mathfrak{g}$ in [8, 9, 10].

\section{SEMI-HOLONOMIC JET MOdUlES AND STRONGLY INVARIANT OPERATORS}

Semi-holonomic jet prolongations of modules were first introduced in the context of AHS-structures in [8]. Here we develop the concept in the more general setting of parabolic geometries and we discuss how the homomorphisms of semi-holonomic jet prolongations give rise to natural operators. Throughout this section, there will be essentially no differences in the arguments for the real and complex parabolic geometries. Thus we shall not mention the field of scalars explicitly, and one has to think of the proper real or complex modules in the applications below.

3.1. The absolutely invariant derivative. Suppose that $(\mathcal{G}, \omega)$ is a parabolic geometry on a manifold $M$. We mentioned in 2.5, that the tangent and cotangent bundles on the homogeneous spaces are homogeneous vector bundles. The Cartan connection $\omega$ extends this identification to all parabolic geometries as follows:

We identify $\mathfrak{g}_{-}$(as a $P$-module) with $\mathfrak{g} / \mathfrak{p}$, and consider the map $\mathcal{G} \times \mathfrak{g}_{-} \rightarrow$ $T M$ defined by mapping $(u, X)$ to $T p \cdot \omega_{u}^{-1}(X)$, where $p: \mathcal{G} \rightarrow M$ is the projection. The equivariancy of the Cartan connection immediately implies that this factors to a vector bundle homomorphism $\mathcal{G} \times_{P} \mathfrak{g}_{-} \rightarrow T M$. Since this is immediately seen to be surjective, it must be an isomorphism of vector bundles by dimensional reasons. Thus we have identified $T M$ with the natural bundle associated to the $P$-module $\mathfrak{g}_{-}$. Now, the invariance of the Killing form on $\mathfrak{g}$ implies that $\mathfrak{g} / \mathfrak{p}$ and $\mathfrak{p}_{+}$with the actions induced by the adjoint action are dual $P$-modules. Thus, similarly as above the cotangent bundle $T^{*} M$ of $M$ can be identified with the bundle $\mathcal{G} \times{ }_{P} \mathfrak{p}_{+}$(implicitly, this has been used in 2.13 above).

There is a nice way to encode the action of vector fields on functions (or equivalently the exterior derivative of functions) using the identifications made above. As we have seen, a typical tangent vector on $M$ can be written as $T p \cdot \omega_{u}^{-1}(X)$ for an element $X \in \mathfrak{g}_{-}$. Acting with this tangent vector on a smooth function $f \in C^{\infty}(M, \mathbb{R})$, we get $\omega_{u}^{-1}(X) \cdot(f \circ p)$. Now, smooth functions on $M$ are in bijective correspondence with smooth $P$-invariant functions on $\mathcal{G}$, the correspondence given by mapping $f$ to $f \circ p$. To any smooth, $P$-invariant function $f$ on $\mathcal{G}$ we associate a function $\nabla^{\omega} f: \mathcal{G} \rightarrow$ $L\left(\mathfrak{g}_{-}, \mathbb{R}\right)$ defined by $\nabla^{\omega} f(u)(X):=\omega_{u}^{-1}(X) \cdot f$. The equivariancy properties of $\omega$ imply that the map $\nabla^{\omega} f$ is $P$-equivariant. Taking into account the above identification of $T^{*} M$ with an associated bundle and of $L\left(\mathfrak{g}_{-}, \mathbb{R}\right) \simeq \mathfrak{p}_{+}$, we see that $\nabla^{\omega} f$ is a one form on $M$, which by definition coincides with $d f$.

The above procedure immediately suggests a generalization. Let $\mathbb{V}$ be any representation of $P$ and let $V M=\mathcal{G} \times{ }_{P} \mathbb{V}$ be the corresponding associated bundle. Then we can identify smooth sections of $V M$ with smooth maps $\mathcal{G} \rightarrow \mathbb{V}$, which are $P$-equivariant. Now to any smooth function $s: \mathcal{G} \rightarrow \mathbb{V}$ 
we associate a smooth function $\nabla^{\omega} s: \mathcal{G} \rightarrow L\left(\mathfrak{g}_{-}, \mathbb{V}\right)$ defined by

$$
\nabla^{\omega} s(u)(X):=\omega_{u}^{-1}(X) \cdot s .
$$

Obviously, this defines a differential operator

$$
C^{\infty}(\mathcal{G}, \mathbb{V}) \rightarrow C^{\infty}\left(\mathcal{G}, L\left(\mathfrak{g}_{-}, \mathbb{V}\right)\right)
$$

and these operators (for all $(\mathcal{G}, \omega)$ ) form a natural operator on all parabolic geometries in the sense of 2.11. This operation is called the universal covariant derivative in the book [31, p. 194]. In [8, 2.3] we have chosen to call it the absolutely invariant derivative. The reason for the latter name also shows the main drawback of this operation: It is not really covariant, i.e. if one starts with an equivariant map $s$ (i.e. a section of $V M$ ) the result is not equivariant in general. Thus in general, if we start with a section, the result of the invariant derivative is not a section of a bundle anymore.

3.2. There is a way, however, to make a section of an associated bundle out of a section of an associated bundle and its absolutely invariant derivative. This is called the invariant one-jet of the section. To describe it, we first have to analyze the action of $G$ on one-jets in the homogeneous case. Thus, let us consider a representation $\mathbb{V}$ of $P$, the corresponding homogeneous bundle $V(G / P)=G \times{ }_{P} \mathbb{V}$ and its first jet prolongation $J^{1}(V(G / P)) \rightarrow G / P$. As we noted in 2.6 this is again a homogeneous bundle, and we want to describe the corresponding action of $P$ on its standard fiber $\mathcal{J}^{1}(\mathbb{V}):=J^{1}(V(G / P))_{o}$. As we noticed in 2.4 it suffices to understand this space as a module over $G_{0}$ and over $\mathfrak{p}_{+}$(in fact, already $\mathfrak{g}_{1}$ would be sufficient).

If we think of sections in $\Gamma(V(G / P))$ as $P$-equivariant functions $s \in$ $C^{\infty}(G, \mathbb{V})^{P}$, then the 1 -jets of sections at the distinguished point $o \in G / P$ are identified with 1 -jets of equivariant functions at the unit $e \in G$ and the action is given by $g \cdot\left(j_{e}^{1} s\right)=j_{e}^{1}\left(s \circ \ell_{g^{-1}}\right)$ for all $g \in G$. Thus, the induced action of $Z \in \mathfrak{p}$ on the section $s$ is given by the differentiation in the direction of the right invariant vector field $R_{Z}$ on $G, Z . j_{e}^{1} s=-j_{e}^{1}\left(R_{Z} \cdot s\right)$.

Now we can identify a one-jet $j_{e}^{1}(s)$ with $(s(e), d s(e))$ and as we saw in 3.1 above, $d s(e)=\nabla^{\omega} s(e)$. As a vector space we can thus write

$$
\mathcal{J}^{1}(\mathbb{V})=\mathbb{V} \oplus\left(\mathfrak{g}_{-}^{*} \otimes \mathbb{V}\right)
$$

and we have to understand the induced actions of $G_{0}$ and $\mathfrak{p}_{+}$on this space. Let us first assume that $g \in G_{0}$. Then $\left(s \circ \ell_{g^{-1}}\right)(e)=s\left(g^{-1}\right)=g \cdot s(e)$ by equivariancy of $s$. On the other hand, we have to evaluate $\omega_{e}^{-1}(X) \cdot\left(s \circ \ell_{g}^{-1}\right)$. This can be computed as

$$
\begin{aligned}
\left.\frac{d}{d t}\right|_{t=0} s\left(g^{-1} \exp (t X)\right)= & \left.\frac{d}{d t}\right|_{t=0} s\left(g^{-1} \exp (t X) g g^{-1}\right)= \\
& =\omega_{e}^{-1}\left(\operatorname{Ad}\left(g^{-1}\right) X\right) \cdot(g \cdot s)=g \cdot\left(\omega_{e}^{-1}\left(\operatorname{Ad}\left(g^{-1}\right) X\right) \cdot s\right) .
\end{aligned}
$$

Now since $g \in G_{0}$, we have $\operatorname{Ad}\left(g^{-1}\right) X \in \mathfrak{g}_{-}$for all $X \in \mathfrak{g}_{-}$(the adjoint action on $\mathfrak{g}_{-}$coincides with the induced action on $\mathfrak{g} / \mathfrak{p}$ in this case), so we see that $\mathcal{J}^{1}(\mathbb{V})=\mathbb{V} \oplus\left(\mathfrak{g}_{-}^{*} \otimes \mathbb{V}\right)$ even as a $G_{0}$-module.

For $Z \in \mathfrak{p}_{+}$we have $-\left(R_{Z} \cdot s\right)(e)=Z \cdot(s(e))$ by the infinitesimal version of equivariancy of $s$. On the other hand, for the derivative component we have to compute the linear mapping $\mathfrak{g}_{-} \ni X \mapsto-\omega^{-1}(X) \cdot R_{Z} \cdot s(e)$. Since $\omega^{-1}(X)$ 
is left invariant, it commutes with $R_{Z}$ and the resulting expression depends only on $R_{Z}(e)=Z=\omega^{-1}(Z)(e)$, and we get

$$
\begin{aligned}
-\omega^{-1}(X) \cdot R_{Z} \cdot s(e) & =-\omega^{-1}(Z) \cdot \omega^{-1}(X) \cdot s(e) \\
& =-\omega^{-1}(X) \cdot \omega^{-1}(Z) \cdot s(e)-\left[\omega^{-1}(Z), \omega^{-1}(X)\right] \cdot s(e) .
\end{aligned}
$$

The infinitesimal version of equivariancy of $s$ shows that the first term in the last expression gives $Z \cdot\left(\omega_{e}^{-1}(X) \cdot s(e)\right)$. Since $\omega^{-1}(-)$ is just the left invariant vector field, the second term gives $-\omega_{e}^{-1}([Z, X]) \cdot s$. Now let us $\operatorname{split} \operatorname{ad}(Z)=$ $\operatorname{ad}_{-}(Z) \oplus \operatorname{ad}_{\mathfrak{p}}(Z)$ according to the splitting $\mathfrak{g}=\mathfrak{g}_{-} \oplus \mathfrak{p}$. Then the $\operatorname{ad}_{\mathfrak{p}}(Z)(X)-$ part acts algebraically by equivariancy of $s$ while the rest simply produces $-\omega_{e}^{-1}\left(\operatorname{ad}_{-}(Z)(X)\right) \cdot s$.

Thus, if we denote elements of $\mathcal{J}^{1}(\mathbb{V})$ as pairs $(v, \varphi)$, where $v \in \mathbb{V}$ and $\varphi$ is a linear map from $\mathfrak{g}_{-}$to $\mathbb{V}$, then the appropriate action of $Z \in \mathfrak{p}_{+}$is given by

$$
Z \cdot(v, \varphi)=\left(Z \cdot v, X \mapsto Z \cdot(\varphi(X))-\varphi\left(\operatorname{ad}_{-}(Z)(X)\right)+\operatorname{ad}_{\mathfrak{p}}(Z)(X) \cdot v\right),
$$

i.e. we get the tensorial action plus one additional term mapping the valuepart to the derivative-part.

This action can also be nicely written in a tensorial notation. To do this let us choose a basis $\left\{\eta_{\alpha}\right\}$ of $\mathfrak{p}_{+}$such that each element $\eta_{\alpha}$ is homogeneous of degree $\left|\eta_{\alpha}\right|$, and let $\left\{\xi_{\alpha}\right\}$ be the dual basis of $\mathfrak{g}_{-}$(with respect to the Killing form $B)$. Now consider an element $\left(v_{0}, Z_{1} \otimes v_{1}\right) \in$ $\mathcal{J}^{1}(\mathbb{V})$, where $v_{0}, v_{1} \in \mathbb{V}$ and $Z_{1} \in \mathfrak{p}_{+} \cong \mathfrak{g}_{-}^{*}$. Then by definition $Z_{1} \otimes v_{1}$ maps $X \in \mathfrak{g}_{-}$to $B(Z, X) v_{1}$. Thus $[Z, X]_{-}:=\operatorname{ad}_{-}(Z)(X)$ is mapped to $B\left(Z_{1},[Z, X]_{-}\right) v_{1}$. Since the Killing form vanishes on $\mathfrak{p}_{+} \times \mathfrak{p}$, this can be rewritten as $B\left(Z_{1},[Z, X]\right) v_{1}=B\left(\left[Z_{1}, Z\right], X\right) v_{1}$. Moreover, we can write $\operatorname{ad}_{Z}$ as an element of $L\left(\mathfrak{g}_{-}, \mathfrak{g}\right) \cong \mathfrak{p}_{+} \otimes \mathfrak{g}$ in the form $\sum_{\alpha} \eta_{\alpha} \otimes\left[Z, \xi_{\alpha}\right]$. This implies that for $Z$ homogeneous of degree $|Z|$, we may rewrite the action on $\mathcal{J}^{1} \mathbb{V}$ as

$$
Z \cdot\left(v_{0}, Z_{1} \otimes v_{1}\right)=\left(Z \cdot v_{0}, Z_{1} \otimes Z \cdot v_{1}+\left[Z, Z_{1}\right] \otimes v_{1}+\sum_{\left|\eta_{\alpha}\right| \leq|Z|} \eta_{\alpha} \otimes\left[Z, \xi_{\alpha}\right] \cdot v_{0}\right) .
$$

A simple computation shows that $\mathcal{J}^{1}(-)$ can be made into a functor on the category of $P$-modules by defining

$$
\mathcal{J}^{1}(f)(v, \varphi):=(f(v), f \circ \varphi)
$$

for each $P$-module homomorphism $f: \mathbb{V} \rightarrow \mathbb{W}$.

3.3. Surprisingly, the first jet prolongation of representations introduced above leads for any parabolic geometry to a natural identification of the first jet prolongation of any natural bundle with an associated bundle, i.e. with another natural bundle. Let $(\mathcal{G}, \omega)$ be a parabolic geometry on $M$, let $\mathbb{V}$ be a representation of $P$, and let $V M$ be the corresponding associated bundle over $M$.

Proposition. The invariant differential $\nabla^{\omega}$ defines the mapping

$$
\iota: C^{\infty}(\mathcal{G}, \mathbb{V})^{P} \rightarrow C^{\infty}\left(\mathcal{G}, \mathcal{J}^{1} \mathbb{V}\right)^{P}, \quad \iota(s)(u)=\left(s(u),\left(X \mapsto \nabla^{\omega} s(u)(X)\right)\right)
$$

which yields an isomorphism $J^{1} V M \simeq \mathcal{G} \times_{P} \mathcal{J}^{1} \mathbb{V}$.

For each fiber bundle map $V M \rightarrow W M$ induced by a $P$-module homomorphism $f: \mathbb{V} \rightarrow \mathbb{W}$, the first jet prolongation of the bundle map is induced by the $P$-module homomorphism $\mathcal{J}^{1}(f)$. 
Proof. Let us recall that $\nabla^{\omega} s(u)(X)=\omega^{-1}(X)(u) \cdot s$. Thus the mapping $\iota: s \mapsto\left(s, \nabla^{\omega} s\right)$ is well defined and depends on first jets only, so we only have to check that the values are actually equivariant. First, for $g \in G_{0}$ we have to compute $\left(s(u \cdot g), \nabla^{\omega} s(u \cdot g)\right)$. Equivariancy of $s$ implies $s(u \cdot g)=g^{-1} \cdot(s(u))$. The second component maps $X \in \mathfrak{g}_{-}$to $\omega_{u \cdot g}^{-1}(X) \cdot s$. Now the equivariancy of $\omega$ immediately implies that $\omega_{u \cdot g}^{-1}(X)=\operatorname{Tr}^{g} \cdot \omega_{u}^{-1}(\operatorname{Ad}(g) X)$. Since $g \in G_{0}$ we see that $\operatorname{Ad}(g) X \in \mathfrak{g}_{-}$and using equivariancy of $s$ again, we see that $\nabla^{\omega} s(u \cdot g)$ maps $X$ to $g^{-1} \cdot\left(\omega_{u}^{-1}(\operatorname{Ad}(g) X) \cdot s\right)$, and thus $\left(s(u \cdot g), \nabla^{\omega} s(u \cdot g)\right)=$ $g^{-1} \cdot\left(s(u), \nabla^{\omega} s(u)\right)$.

On the other hand, we have to check equivariancy for the infinitesimal action of $Z \in \mathfrak{p}_{+}$. Thus, we have to compute $\left(\left(\zeta_{Z} \cdot s\right)(u), \zeta_{Z} \cdot\left(\nabla^{\omega} s\right)(u)\right)$. Equivariancy of $s$ implies that the first component equals $-Z \cdot(s(u))$. The second component maps $X \in \mathfrak{g}_{-}$to $\left(\zeta_{Z} \cdot \omega^{-1}(X) \cdot s\right)(u)$. Now $\zeta_{Z}=\omega^{-1}(Z)$ and we can rewrite the expression as

$$
\left(\omega^{-1}(X) \cdot \omega^{-1}(Z) \cdot s\right)(u)+\left[\omega^{-1}(Z), \omega^{-1}(X)\right] \cdot s(u) .
$$

Since the curvature of $\omega$ is horizontal and $\omega^{-1}(Z)$ is vertical, we may rewrite the second term in this expression as $\left(\omega^{-1}([Z, X]) \cdot s\right)(u)$. Now we can split $[Z, X]$ into a $\mathfrak{g}_{-}$and a $\mathfrak{p}$-component and conclude as in 3.2 above that $\left(\left(\zeta_{Z} \cdot s\right)(u), \zeta_{Z} \cdot\left(\nabla^{\omega} s\right)(u)\right)=-Z \cdot\left(s(u), \nabla^{\omega} s(u)\right)$.

Clearly, this construction gives a smooth injective homomorphism of vector bundles $J^{1} V M \rightarrow \mathcal{G} \times{ }_{P} \mathcal{J}^{1} \mathbb{V}$, which covers the identity map on $M$. Since both bundles clearly have the same rank, this must be an isomorphism.

Finally, consider a homomorphism $f: \mathbb{V} \rightarrow \mathbb{W}$. The corresponding bundle map $V M \rightarrow W M$ is induced by $(u, v) \mapsto(u, f(v))$, and so the induced action on sections is induced by

$$
s \mapsto(x \mapsto(u(x), f \circ s(u(x)))) .
$$

Taking 1-jet of this expression we obtain just the homomorphism $\mathcal{J}^{1}(f)$.

3.4. Semi-holonomic jets. Since we posed no conditions on the representation $\mathbb{V}$ above, we can iterate the functors $J^{1}$ on the associated vector bundles as well as the functors $\mathcal{J}^{1}$ on the $P$-modules. Proposition 3.3 then implies that the $r$-th iteration $J^{1} \ldots J^{1} V M$ is an associated bundle to $\mathcal{G}$ corresponding to the $P$-module $\mathcal{J}^{1} \ldots \mathcal{J}^{1} \mathbb{V}$. Let us look more carefully at $\mathcal{J}^{1} \mathcal{J}^{1} \mathbb{V}$ and $J^{1} J^{1} V M$. There are two obvious $P$-module homomorphisms $\mathcal{J}^{1} \mathcal{J}^{1} \mathbb{V} \rightarrow \mathcal{J}^{1} \mathbb{V}$, the first one given by the projection $p_{\mathcal{J}^{1} \mathbb{V}}$ defined on each first jet prolongation by projection to the first component, and the other one obtained by the action of $\mathcal{J}^{1}$ on $p_{\mathbb{V}}$. Thus there is the submodule $\overline{\mathcal{J}}^{2} \mathbb{V}$ in $\mathcal{J}^{1} \mathcal{J}^{1} \mathbb{V}$ on which these two projections coincide. As a vector space and a $G_{0}$-module we have

$$
\overline{\mathcal{J}}^{2} \mathbb{V}=\mathbb{V} \oplus\left(\mathfrak{g}_{-}^{*} \otimes \mathbb{V}\right) \oplus\left(\mathfrak{g}_{-}^{*} \otimes \mathfrak{g}_{-}^{*} \otimes \mathbb{V}\right)
$$

The two $P$-module homomorphisms $\mathcal{J}^{1}\left(p_{\mathbb{V}}\right)$ and $p_{\mathcal{J}^{1} \mathbb{V}}$ give rise to vector bundle homomorphisms $J^{1} J^{1} V M \rightarrow J^{1} V M$ which are just the two standard projections on the second non-holonomic jet prolongation. So we conclude that the second semi-holonomic prolongation $\bar{J}^{2} V M$ is naturally isomorphic to $\mathcal{G} \times_{P} \overline{\mathcal{J}}^{2} \mathbb{V}$.

Iterating this procedure, we obtain the $r$-th semi-holonomic jet prolongations and $\mathcal{J}^{1}\left(\overline{\mathcal{J}}^{r} \mathbb{V}\right)$ equipped with two natural projections onto $\mathcal{J}^{1}\left(\overline{\mathcal{J}}^{r-1} \mathbb{V}\right)$, 
which correspond to the usual projections on the first jet prolongation of semi-holonomic jets. Their equalizer is then the submodule $\overline{\mathcal{J}}^{r+1} \mathbb{V}$. As a $G_{0}$-module

$$
\overline{\mathcal{J}}^{r} \mathbb{V}=\bigoplus_{i=0}^{r}\left(\otimes^{i} \mathfrak{g}_{-}^{*} \otimes \mathbb{V}\right) .
$$

Proposition. For each positive integer $r$, the $r$-th semi-holonomic jet prolongation $\bar{J}^{r} V M$ carries the natural structure of associated vector bundle $\mathcal{G} \times_{P} \overline{\mathcal{J}}^{r} \mathbb{V}$. Moreover, there is the natural embedding

$$
\begin{gathered}
J^{r} V M \rightarrow \bar{J}^{r} V M \simeq \mathcal{G} \times{ }_{P} \overline{\mathcal{J}}^{r} \mathbb{V} \\
j^{r} s(u) \mapsto\left\{u,\left(s(u), \nabla^{\omega} s(u), \ldots,\left(\nabla^{\omega}\right)^{r} s(u)\right)\right\} .
\end{gathered}
$$

Proof. The first part of the statement has been already shown. What remains is to discuss the equivariancy properties of the invariant differentials. However also this follows from the first order case easily by induction, using only the definition of the semi-holonomic prolongations.

3.5. Strongly invariant operators. The problem, why we cannot work with true (holonomic) $r$-jets but have to use the semi-holonomic ones, is that absolutely invariant derivatives commute only for flat Cartan connections. More precisely, from the definition of the absolutely invariant derivative and the properties of the curvature, one immediately concludes the so called general Ricci-identity

$$
\begin{aligned}
\left(\nabla^{\omega} \nabla^{\omega} s\right)(u)(X \otimes Y-Y \otimes X)= & \nabla^{\omega} s(u)([X, Y])+\kappa_{\mathfrak{p}}(X, Y) \cdot(s(u)) \\
& -\nabla^{\omega} s(u)\left(\kappa_{-}(X, Y)\right)
\end{aligned}
$$

for all $X, Y \in \mathfrak{g}_{-}$. This also shows that the torsion-part of $\kappa$ has a quite different geometric meaning than the component valued in $\mathfrak{p}$. Thus, the identification from proposition 3.4 has values in the $P$-submodule $\mathcal{J}^{r}(\mathbb{V})$ of symmetric elements $\oplus_{i=0}^{r}\left(S^{i} \mathfrak{g}_{-}^{*} \otimes \mathbb{V}\right)$ in the flat case. Consequently we have recovered the standard identification of the $r$-th holonomic jet prolongation of a homogeneous bundle with an associated bundle for flat geometries, but this does not work in the curved case.

Nevertheless, one can well use the semi-holonomic jet prolongations to generate invariant operators. Suppose that $\mathbb{V}$ and $\mathbb{W}$ are representations of $P$ and suppose that $\Phi: \overline{\mathcal{J}}^{r}(\mathbb{V}) \rightarrow \mathbb{W}$ is a homomorphism of $P$-modules. Then for any parabolic geometry $(\mathcal{G}, \omega)$ we can define a differential operator $\Gamma(V M) \rightarrow \Gamma(W M)$ as follows: For a section $s$ viewed as an equivariant function $\mathcal{G} \rightarrow \mathbb{V}$ define

$$
D_{(\mathcal{G}, \omega)}(s)(u)=\Phi\left(s(u), \nabla^{\omega} s(u), \ldots,\left(\nabla^{\omega}\right)^{r} s(u)\right) .
$$

From Proposition 3.4 above it follows that this gives a section of the bundle $W M$ and that each $D_{(\mathcal{G}, \omega)}$ is a differential operator of order $\leq r$. Moreover, by construction the operators $D_{(\mathcal{G}, \omega)}$ form a natural operator on the category of all parabolic geometries in the sense of 2.11. Operators arising in this way will be called strongly invariant operators in the sequel. We will often not distinguish carefully between a strongly invariant operator and the corresponding homomorphism $\overline{\mathcal{J}}^{r}(\mathbb{V}) \rightarrow \mathbb{W}$. Thus, the semi-holonomic jet modules give a possibility to construct natural operators for a parabolic 
geometry in a completely algebraic way, since one only has to construct a homomorphism between two finite dimensional $P$-modules.

There is a slight problem about strongly invariant operators, however. Namely, even if a homomorphism $\overline{\mathcal{J}}^{r}(\mathbb{V}) \rightarrow \mathbb{W}$ does not factor over $\overline{\mathcal{J}}^{r-1}(\mathbb{V})$, the corresponding operators may be of order strictly less than $r$ or even identically zero. To see this, note that we can easily compute the symbol of a strongly invariant operator. This symbol is a vector bundle homomorphism $S^{r} T^{*} M \otimes V M \rightarrow W M$, which is induced by a homomorphism $S^{r} \mathfrak{g}_{-}^{*} \otimes \mathbb{V} \rightarrow$ $\mathbb{W}$. Using Proposition 3.4 it is clear that this homomorphism is given by restricting $\Phi$ to $S^{r} \mathfrak{g}_{-}^{*} \otimes \mathbb{V}$, viewed as a submodule of $\otimes^{r} \mathfrak{g}_{-}^{*} \otimes \mathbb{V}$, which in turn can be viewed as a submodule of $\overline{\mathcal{J}}^{r}(\mathbb{V})$. Thus, if a homomorphism restricts to zero on the symmetric part of the top component of the jetmodule, then the corresponding operator actually is of lower order (and contains terms involving the curvature of the Cartan connection).

There is an important situation in which this problem does not play any role. Suppose that we have an operator of order $r$ in the flat case with nontrivial symbol, and suppose that we can find a homomorphism $\overline{\mathcal{J}}^{r}(\mathbb{V}) \rightarrow$ $\mathbb{W}$ which induces this operator (in the flat case). Then this gives a curved analog of the operator in question, and there is no problem with the symbol at all. This will always be the case for the operators we are going to study. In particular, since $\overline{\mathcal{J}}^{1}(\mathbb{V})=\mathcal{J}^{1}(\mathbb{V})$, any first order invariant operator on the category of flat parabolic geometries is automatically strongly invariant, and thus has a canonical curved analog.

3.6. Remark. There are operators which are natural (invariant) in the sense of 2.11 but are not strongly invariant. Basically, there is only one example of such an operator known: It is shown in [21] that on conformal manifolds of dimension $2 m$ there exists a conformally invariant $m$-th power of the Laplacian on smooth functions. In [16] it is shown that this operator is not strongly invariant. It can, however, be written in terms of absolutely invariant derivatives, and thus it is also natural. In fact, it is shown in [32] that for AHS-structures, i.e. parabolic geometries corresponding to $|1|-$ graded Lie algebras, naturality of (even non-linear) operators is equivalent to the possibility to express them by means of the absolute invariant derivative and curvature of the defining Cartan connection, and this, in turn, is equivalent to the existence of a universal formula in terms of all underlying affine connections, cf. 2.13.

The existence of invariant operators which are not strongly invariant is due to symmetries of the curvature of a Cartan connection. Suppose that we write an expression in terms of absolutely invariant derivatives and check whether the result is $P$-equivariant. Otherwise put, we can compute the obstruction against being equivariant which usually contains expressions involving the curvature of the Cartan connection and its derivatives. In the case of a strongly invariant operator, these obstructions vanish algebraically. But the jets of the curvature of any Cartan connection have certain symmetries, basically due to the Bianchi identity, see e.g. [7, 4.9]. This implies that expressions that do not vanish algebraically, still may vanish whenever the jet of the curvature of a Cartan connection is inserted, and this is precisely what happens in the case of the critical powers of the Laplacian. 
3.7. Twisted invariant operators. Besides the completely reducible representations (which come from the reductive subgroup $G_{0}$ ) there is a second class of particularly simple representations of the group $P$. Namely one can take a representation of the full (semisimple) group $G$ and restrict it to $P$. These representations have particularly nice features in the case of the flat model since they give rise to trivial homogeneous bundles. There are many ways to see that, but the most appropriate one for our purposes is to associate to any element $v$ in a representation $\mathbb{V}$ of $G$ a global nonzero section of the associated bundle $G \times_{P} \mathbb{V}$. To do this, we just have to specify a $P$ equivariant map $G \rightarrow \mathbb{V}$, and we define this map simply by $g \mapsto g^{-1} \cdot v$. This map is even $G$-equivariant and not only $P$-equivariant.

There is a simple generalization of this result. Suppose that $\mathbb{W}$ is any representation of $P$. Then sections of $W(G / P)$ are in bijective correspondence with $P$-equivariant maps $G \rightarrow \mathbb{W}$. Now we define a map on sections of homogeneous bundles

$$
\begin{gathered}
\Gamma(W(G / P)) \otimes \mathbb{V} \rightarrow \Gamma(W(G / P) \otimes V(G / P)) \\
s \otimes v \mapsto\left(g \in G \mapsto s(g) \otimes g^{-1} \cdot v\right)
\end{gathered}
$$

and one immediately verifies that this is an isomorphism of $G$-modules. In particular, this implies that if $\mathbb{W}^{\prime}$ is another $P$-representation and $D$ : $\Gamma(W(G / P)) \rightarrow \Gamma\left(W^{\prime}(G / P)\right)$ is an invariant differential operator, then we can pull back

$$
D \otimes \operatorname{id}_{\mathbb{V}}: \Gamma(W(G / P)) \otimes \mathbb{V} \rightarrow \Gamma\left(W^{\prime}(G / P)\right) \otimes \mathbb{V}
$$

along these isomorphisms to get an invariant operator

$$
D_{\mathbb{V}}: \Gamma(W(G / P) \otimes V(G / P)) \rightarrow \Gamma\left(W^{\prime}(G / P) \otimes V(G / P)\right) .
$$

This operator is called the twisted invariant operator corresponding to $D$ and $\mathbb{V}$.

Now, let us notice that the above isomorphism between the spaces of sections of the associated bundles induces a $P$-module isomorphism $\overline{\mathcal{J}}^{r}(\mathbb{W}) \otimes$ $\mathbb{V} \simeq \overline{\mathcal{J}}^{r}(\mathbb{W} \otimes \mathbb{V})$ for all $P$-modules $\mathbb{W}$ and $G$-modules $\mathbb{V}$ and all orders $r$. Thus, for strongly invariant operators $D$, we may extend the construction of the twisted invariant operators to natural operators $D_{\mathbb{V}}$ acting on all geometries $(\mathcal{G}, \omega)$ of the type $(G, P)$ and the resulting operators are again strongly invariant. Let us remark that a completely algebraic treatment of this construction has been worked out (in the special case of the AHS-structures) in 6.

In particular, we obtain the strongly invariant twisted operators $D_{\mathbb{V}}$ for all first order invariant operators $D$ on the homogeneous vector bundles and all $G$-modules $\mathbb{V}$.

3.8. Twisted exterior derivatives. The standard exterior derivatives $d$ on the differential forms on $G / P$ are first order invariant operators (since they are even invariant under the action of all diffeomorphisms of $G / P$ ), so we can apply the construction above to get the twisted exterior derivatives

$$
d_{\mathbb{V}}: \Gamma\left(\Lambda^{n} T^{*}(G / P) \otimes V(G / P)\right) \rightarrow \Gamma\left(\Lambda^{n+1} T^{*}(G / P) \otimes V(G / P)\right)
$$

for $n=0, \ldots, \operatorname{dim}(G / P)$. Moreover, the operators $d_{\mathbb{V}}$ are strongly invariant, since they are of first order, and so there are the corresponding $P$-module 
homomorphisms on the semi-holonomic jet modules. Since we will need it later, we will compute these homomorphisms explicitly.

Let us start with the ordinary exterior derivative. We have already noted in 3.1 that the exterior derivative of functions equals the absolutely invariant derivative. To compute the exterior derivative for general differential forms, we first have to describe nicely the identification of $n$-forms with smooth equivariant functions $G \rightarrow \Lambda^{n} \mathfrak{p}_{+}$. Throughout, we are going to identify $\Lambda^{n} \mathfrak{p}_{+}$ with the space of $n$-linear alternating maps from $\mathfrak{g}_{-} \cong \mathfrak{g} / \mathfrak{p}$ to $\mathbb{K}$. Now using the identification of the tangent bundle of $G / P$ with $G \times{ }_{P} \mathfrak{g}_{-}$described in 3.1, one easily verifies that the relation between a form $\varphi \in \Omega^{k}(G / P)$ and the corresponding function $s: G \rightarrow \Lambda^{n} \mathfrak{p}_{+}$is given by

$$
\left(p^{*} \varphi\right)(g)\left(\omega_{g}^{-1}\left(X_{1}\right), \ldots, \omega_{g}^{-1}\left(X_{n}\right)\right)=s(g)\left(X_{1}, \ldots, X_{n}\right),
$$

where $p^{*} \varphi$ is the pullback of $\varphi$ along the projection $p: G \rightarrow G / P$, and the $X_{i}$ are in $\mathfrak{g}_{-}$. Note that this formula remains correct for $X_{i} \in \mathfrak{g}$ if one interprets $s(g)$ as an $n$-linear map on $\mathfrak{g}$ which vanishes if at least one argument lies in $\mathfrak{p}$.

Lemma. Let $s$ and $d s$ be the functions on $G$ corresponding to differential forms $\varphi$ and $d \varphi$ on $G / P$, respectively. Then the formula for the exterior derivative reads as

$$
\begin{aligned}
d s\left(X_{0}, \ldots, X_{n}\right)= & \sum_{i=0}^{n}(-1)^{i}\left(\nabla^{\omega} s\right)(g)\left(X_{i}\right)\left(X_{0}, \ldots, \hat{i}, \ldots, X_{n}\right)+ \\
& \sum_{i<j}(-1)^{i+j} s(g)\left(\left[X_{i}, X_{j}\right], X_{0}, \ldots, \hat{i}, \ldots, \hat{j}, \ldots, X_{n}\right)
\end{aligned}
$$

where $\omega$ is the left Maurer-Cartan form on $G$ and, as usual, the hat denotes omission.

Proof. To compute the function corresponding to $d \varphi$, we just have to evaluate $p^{*}(d \varphi)(g)=d\left(p^{*} \varphi\right)(g)$ on vector fields of the form $\tilde{X}(g)=\omega_{g}^{-1}(X)$. We have

$$
\begin{array}{r}
d\left(p^{*} \varphi\right)\left(\tilde{X}_{0}, \ldots, \tilde{X}_{n}\right)=\sum_{i=0}^{n}(-1)^{i} \tilde{X}_{i} \cdot\left(p^{*} \varphi\right)\left(\tilde{X}_{0}, \ldots, \hat{i}, \ldots \tilde{X}_{n}\right)+ \\
+\sum_{i<j}(-1)^{i+j}\left(p^{*} \varphi\right)\left(\left[\tilde{X}_{i}, \tilde{X}_{j}\right], \tilde{X}_{0}, \ldots, \hat{i}, \ldots, \hat{j}, \ldots, \tilde{X}_{n}\right) .
\end{array}
$$

Inserting $p^{*} \varphi$ from above and evaluating at $g$, we see directly that the first summand agrees with the first summand in the claimed formula, which clearly equals $n+1$ times the alternation of $\left(\nabla^{\omega} s\right)(g)$ evaluated at $\left(X_{0}, \ldots, X_{n}\right)$.

For the second summand, we just have to note that by the Maurer-Cartan equation for $\omega$ we have $\left[\tilde{X}_{i}, \tilde{X}_{j}\right]=\left[\widehat{X_{i}, X_{j}}\right]$. Thus, this summand gives exactly the other part of the required formula.

Now let us pass to the general case of a $V(G / P)$-valued $n$-form, where $\mathbb{V}$ is a representation of the whole group $G$. Any such form can be written as a finite sum of expressions of the form $\varphi \otimes \tilde{v}$, where $\varphi \in \Omega^{n}(G / P)$ and $\tilde{v}$ is the global section of $V(G / P)$ corresponding to $v \in \mathbb{V}$ as in 3.7 above. By definition, the twisted exterior derivative is given by $d_{\mathbb{V}}(\varphi \otimes \tilde{v})=(d \varphi) \otimes \tilde{v}$. 
Now let $s$ be the function corresponding to $\varphi$ and denote by $\tilde{v}$ also the function corresponding to the global section. From above, we thus see that $d_{\mathbb{V}}(\varphi \otimes \tilde{v})$ is represented by the function which maps $\left(X_{0}, \ldots, X_{n}\right)$ to

$$
\begin{aligned}
(*) \sum_{i=0}^{n}(-1)^{i}\left(\nabla^{\omega} s\right)(g)\left(X_{i}\right)\left(X_{0}, \ldots, \hat{i}, \ldots, X_{n}\right) \tilde{v}(g)+ \\
+\sum_{i<j}(-1)^{i+j} s(g)\left(\left[X_{i}, X_{j}\right], X_{0}, \ldots, \hat{i}, \ldots, \hat{j}, \ldots, X_{n}\right) \tilde{v}(g) .
\end{aligned}
$$

By definition of the absolutely invariant derivative, we have

$$
\nabla^{\omega}(s \otimes \tilde{v})(X)=\nabla^{\omega} s(X) \otimes \tilde{v}+s \otimes\left(\nabla^{\omega} \tilde{v}(X)\right)
$$

and the infinitesimal version of $G$-invariance of $\tilde{v}$ says that

$$
\nabla^{\omega} \tilde{v}(g)(X)=-X \cdot(\tilde{v}(g)) .
$$

Thus we may rewrite the first summand in $(*)$ as

$$
\begin{aligned}
(* *) \quad \sum_{i=0}^{n}(-1)^{i} \nabla^{\omega}(s \otimes \tilde{v})(g)( & \left.X_{i}\right)\left(X_{0}, \ldots, \hat{i}, \ldots, X_{n}\right)+ \\
& +\sum_{i=0}^{n}(-1)^{i} X_{i} \cdot\left(s(g)\left(X_{0}, \ldots, \hat{i}, \ldots, X_{n}\right) \tilde{v}(g)\right) .
\end{aligned}
$$

Finally note that the second term in $(* *)$ adds up with the second term in $(*)$ to the value of the standard Lie algebra differential $\partial: C^{n}\left(\mathfrak{g}_{-}, \mathbb{V}\right)=$ $\Lambda^{n} \mathfrak{g}_{-}^{*} \otimes \mathbb{V} \rightarrow C^{n+1}\left(\mathfrak{g}_{-}, \mathbb{V}\right)$ (cf. 4.1 for the explicit formula) applied to the map $s(g) \otimes \tilde{v}(g)$ evaluated on $\left(X_{0}, \ldots, X_{n}\right)$. Thus we may summarize:

3.9. Proposition. The twisted exterior derivative $d_{\mathbb{V}}$ on $G / P$ is a strongly invariant operator induced by the $P$-module homomorphism $\overline{\mathcal{J}}^{1}\left(\Lambda^{n} \mathfrak{p}_{+} \otimes\right.$ $\mathbb{V}) \rightarrow \Lambda^{n+1} \mathfrak{p}_{+} \otimes \mathbb{V}$, which is given by the formula

$$
\left(f_{0}, Z \otimes f_{1}\right) \mapsto \partial\left(f_{0}\right)+(n+1) Z \wedge f_{1},
$$

where we view elements of $\Lambda^{n} \mathfrak{p}_{+} \otimes \mathbb{V}$ as $n$-linear alternating maps from $\mathfrak{g}_{-}$to $\mathbb{V}$ and $Z \wedge f_{1}$ denotes the alternation of the map $\left(X_{0}, \ldots, X_{n}\right) \mapsto$ $B\left(Z, X_{0}\right) f_{1}\left(X_{1}, \ldots, X_{n}\right)$.

3.10. Corollary. The Lie algebra differential $\partial$ satisfies

$$
(W \cdot \partial(f)-\partial(W \cdot f))=(n+1) \sum_{\left|\eta_{\alpha}\right| \leq|W|} \eta_{\alpha} \wedge\left[W, \xi_{\alpha}\right] \cdot f
$$

for $f \in \Lambda^{n} \mathfrak{p}_{+} \otimes \mathbb{V}$ and $W \in \mathfrak{p}_{+}$, where $\xi_{\alpha}$ and $\eta_{\alpha}$ are homogeneous dual bases of $\mathfrak{g}_{-}$and $\mathfrak{p}_{+}$with respect to the Killing form.

Proof. The claim can be verified by a nice and elementary, but tedious algebraic computation. However, the previous proposition offers the following simple argument:

We know that the formula for the strongly invariant operator

$$
d_{\mathbb{V}}\left(f_{0}, Z \otimes f_{1}\right)=\partial\left(f_{0}\right)+(n+1) Z \wedge f_{1}
$$


is $P$-equivariant. Thus for all $f_{0}, f_{1} \in \mathbb{V}, Z \in \mathfrak{p}_{+}, W \in \mathfrak{p}_{+}$we obtain the equality of the following two expressions

$$
\begin{gathered}
d_{\mathbb{V}}\left(W \cdot\left(f_{0}, Z \otimes f_{1}\right)\right)=d_{\mathbb{V}}\left(\left(W \cdot f_{0}, W \cdot\left(Z \otimes f_{1}\right)+\sum \eta_{\alpha} \otimes\left[W, \xi_{\alpha}\right] \cdot f_{0}\right)=\right. \\
=\partial\left(W \cdot f_{0}\right)+(n+1) W \cdot\left(Z \wedge f_{1}\right)+(n+1) \sum \eta_{\alpha} \wedge\left[W, \xi_{\alpha}\right] \cdot f_{0} \\
W \cdot\left(\partial\left(f_{0}\right)+(n+1) Z \wedge f_{1}\right)=W \cdot\left(\partial f_{0}\right)+(n+1) W \cdot\left(Z \wedge f_{1}\right) .
\end{gathered}
$$

This yields the required formula.

3.11. The covariant exterior derivatives. Proposition 3.9 offers a canonical curved analog of the twisted exterior derivatives on all manifolds with a parabolic geometry of the type $(G, P)$. It should be remarked that we may obtain another curved analog as follows. For any parabolic geometry $(\mathcal{G}, \omega)$ on $M$, we consider the extended bundle $\tilde{\mathcal{G}}=\mathcal{G} \times{ }_{P} G$, which is a principal $G$-bundle over $M$. It is a classical observation that the Cartan connection $\omega$ induces a principal connection $\tilde{\omega}$ on $\tilde{\mathcal{G}}$. Now if $\mathbb{V}$ is a representation of $G$, then we can view the corresponding natural bundle $V M=\mathcal{G} \times{ }_{P} \mathbb{V}$ also as $V M=\tilde{\mathcal{G}} \times{ }_{G} \mathbb{V}$, and thus we have the induced linear connection on this bundle. The covariant exterior derivative with respect to this connection gives a natural operator on $V M$-valued forms on $M$. If $s: \tilde{\mathcal{G}} \rightarrow \Lambda^{k} \mathfrak{p}_{+} \otimes \mathbb{V}$ is the equivariant function corresponding to a $k$-form $\varphi$ on $M$, then the value of the latter operator is a $(k+1)$-form on $M$, given by the formula

$$
\begin{array}{r}
d^{\tilde{\omega}} s(u)\left(X_{0}, \ldots, X_{n}\right)=\sum_{i=0}^{k}(-1)^{i} \nabla_{X_{i}}^{\tilde{\omega}} s(u)\left(X_{0}, \ldots, \hat{i}, \ldots, X_{k}\right)+ \\
+\sum_{i<j}(-1)^{i+j} s(u)\left(\left[X_{i}, X_{j}\right], X_{0}, \ldots, \hat{i}, \ldots, \hat{j}, \ldots, X_{k}\right)
\end{array}
$$

where $X_{0}, \ldots, X_{k} \in \mathfrak{g}_{-}, u \in \tilde{\mathcal{G}}, \nabla_{X_{i}}^{\tilde{\omega}} s(u)$ means the derivative of $s$ in the direction of the horizontal vector at $u$ determined by $X_{i}$, and there are the standard omissions of arguments in the expressions on the right hand side. Indeed, $d^{\tilde{\omega}}$ is defined as the pullback of the standard $d$ on $\tilde{\mathcal{G}}$ by the horizontal projection of $\tilde{\omega}$, applied to the pullback of the $k$-form $\varphi$ on $M$ by the projection $p: \tilde{\mathcal{G}} \rightarrow M$. Since the curvature of $\tilde{\omega}$ produces vertical fields on $\tilde{\mathcal{G}}$, the above formula equals to the standard evaluation of $d\left(p^{*} \varphi\right)$ on the horizontal lifts of vector fields on $M$.

These operators coincide with the twisted exterior derivatives on the homogeneous space but they differ in general. The explicit general comparison is as follows:

Lemma. Let $\mathbb{V}$ be a $G$-module, $V M$ the corresponding natural vector bundle over a manifold $M$ equipped with a parabolic geometry $(\mathcal{G}, \omega)$. The covariant exterior derivative $d^{\tilde{\omega}}$ on $\Lambda^{k} T^{*} M \otimes V M, k>0$, and the twisted exterior derivative $d_{\mathbb{V}}$ on the same space satisfy

$$
d^{\tilde{\omega}} \varphi=d_{\mathbb{V}} \varphi+i_{\kappa_{-}} \varphi
$$

where $\kappa_{-}$is the torsion-component of the curvature of $\omega$ and $i_{\kappa_{-}} \varphi$ is the usual insertion operator evaluated on $\kappa_{-}$and $\varphi$, i.e. the alternation of $\varphi\left(\kappa_{-}\left(X_{0}, X_{1}\right), X_{2}, \ldots, X_{k}\right)$ over the arguments. 
Proof. The key to the required formula is in the expressions $(*)$ and $(* *)$ in 3.8. Namely, the latter expressions which were derived on the homogeneous spaces describe also the twisted exterior derivatives in general, but we have to be aware that instead of the bracket $\left[X_{i}, X_{j}\right]$ in $(*)$ we have to plug in

$$
\omega(u)\left(\left[\omega^{-1}\left(X_{i}\right), \omega^{-1}\left(X_{j}\right)\right]\right)=\left[X_{i}, X_{j}\right]-\kappa(u)\left(X_{i}, X_{j}\right) .
$$

At the same time, for all $u \in \mathcal{G} \subset \tilde{\mathcal{G}}$, the covariant derivative $\nabla^{\tilde{\omega}}$ of a section $s: \tilde{\mathcal{G}} \rightarrow \mathbb{V}$ relates to the absolute invariant derivative as

$$
\nabla^{\tilde{\omega}} s(u)(X)=\nabla^{\omega} s(u)(X)+X \cdot s(u)
$$

(since the horizontal fields given by $\tilde{\omega}$ equal to $\omega^{-1}(X)$ minus the fundamental field $\zeta_{X}$ ).

Combining the latter two facts, we see that exactly the expression

$$
\begin{aligned}
& i_{\kappa_{-}} \varphi(u)\left(X_{0}, \ldots, X_{k}\right)= \\
& \sum_{i<j}(-1)^{i+j} \varphi(u)\left(\kappa_{-}\left(X_{i}, X_{j}\right), X_{0}, \ldots, \hat{i}, \ldots, \hat{j}, \ldots, X_{k}\right)
\end{aligned}
$$

has to be added to $d_{\mathbb{V}}(u) \varphi\left(X_{0}, \ldots, X_{k}\right)$ in order to obtain the covariant derivative. This is exactly the evaluation of the insertion operator, cf. 24, $8.2]$.

The latter lemma shows that our twisted exterior differentials $d_{\mathbb{V}}$ are certain torsion adjusted versions of the standard covariant exterior derivatives. In particular, even in the case $\mathbb{V}=\mathbb{R}$ the twisted derivative $d_{\mathbb{R}}$ equals to the usual exterior derivative $d$ if and only if the geometry is torsion-free.

3.12. Remarks. (1) As we saw in 3.8, the isomorphism

$$
\Gamma(W(G / P)) \otimes \mathbb{V} \cong \Gamma(W(G / P) \otimes V(G / P))
$$

of $G$-modules induces an isomorphism of $P$-modules $\overline{\mathcal{J}}^{r}(\mathbb{W}) \otimes \mathbb{V} \cong \overline{\mathcal{J}}^{r}(\mathbb{W} \otimes$ $\mathbb{V}$ ) for any $P$-representation $\mathbb{W}$ and $G$-representation $\mathbb{V}$. This can also be proved algebraically along the lines of [6]. This isomorphism can then be used to define twisted versions of any strongly invariant operators in a completely algebraic way. Using this picture, the subsequent developments in this paper can be viewed as a curved analog of the Jantzen-Zuckermann translation principle in representation theory. The first version of such a curved translation procedure appeared in the context of 4-dimensional conformal geometry in [15], see also [12].

(2) The twisted exterior derivatives give a sequence

$$
\Gamma(V M) \rightarrow \Omega^{1}(M ; V M) \rightarrow \cdots \rightarrow \Omega^{\max }(M ; V M) \rightarrow 0,
$$

of invariant differential operators, where sections and forms are smooth in the real case and holomorphic in the complex case. In the case of the flat model, this sequence is just the pullback of the tensor product of the (smooth or holomorphic) de Rham sequence with $\mathbb{V}$, so it is a resolution of the constant sheaf $\mathbb{V}$. In the case of a general parabolic geometry, it fails to be a complex. Actually, it is easy to verify that the composition $d_{\mathbb{V}} \circ d_{\mathbb{V}}$ is just given by the action of the curvature of $\omega$. Thus, in the case of a flat parabolic geometry, we still get a complex, which by Lemma 3.11 coincides with the complex given by the covariant exterior derivative with respect to the flat 
linear connection induced by the Cartan connection. Note however, that on a flat parabolic geometry bundles corresponding to representation of $G$ are no more trivial in general.

(3) As a $G_{0}$-module, one can split any representation $\mathbb{W}$ of $P$ as $\oplus \mathbb{W}_{j}$ according to eigenvalues of the grading element $E \in \mathfrak{g}_{0}$. Clearly, the action of $\mathfrak{p}_{+}$maps $\mathfrak{g}_{i} \otimes \mathbb{W}_{j}$ to $\mathbb{W}_{j+i}$. In particular, we can apply this to $\Lambda^{n} \mathfrak{p}_{+} \otimes \mathbb{V}$ to split the space $\Omega^{n}(M ; V M)$ into homogeneous components, and analyze how the twisted exterior derivative behaves with respect to this splitting. From the formula in Proposition 3.9 it is obvious that $d_{\mathbb{V}}$ never lowers homogeneous degree and the component of the same homogeneous degree as the input is just the Lie algebra differential $\partial$ composed with the given form. Thus, the homogeneous component of degree zero of $d \mathbb{V}$ is algebraic and equals $\partial$. This observation is crucial for the subsequent development. Using the fact that the Lie algebra cohomology of $\mathfrak{g}_{-}$with coefficients in $\mathfrak{g}$ admits a Hodge theory (which we will discuss in the next section), we will show that we can replace the sequence of remark (2) above by a different sequence in which only sections of completely reducible bundles occur, and which is a complex computing the same cohomology if the original sequence was a complex.

\section{Curved analogs of Bernstein-Gelfand-Gelfand Resolutions}

In this section, we first discuss the Hodge-structure on the standard complex for the cohomology $H^{*}\left(\mathfrak{g}_{-}, \mathbb{V}\right)$ for a $\mathfrak{g}$-module $\mathbb{V}$. Then we come to the core of the paper, the construction of a huge class of distinguished natural operators on all parabolic geometries.

4.1. We have already mentioned the standard complex for the cohomology $H^{*}\left(\mathfrak{g}_{-}, \mathbb{V}\right)$ in 3.8. The chain groups in this complex are the groups $C^{n}\left(\mathfrak{g}_{-}, \mathbb{V}\right)=\Lambda^{n} \mathfrak{g}_{-}^{*} \otimes \mathbb{V}$, which are viewed as the spaces of $n$-linear alternating maps from $\mathfrak{g}_{-}$to $\mathbb{V}$. The differential

$$
\partial: C^{n}\left(\mathfrak{g}_{-}, \mathbb{V}\right) \rightarrow C^{n+1}\left(\mathfrak{g}_{-}, \mathbb{V}\right)
$$

is defined by

$$
\begin{aligned}
\partial(f)\left(X_{0}, \ldots, X_{n}\right) & =\sum_{i=0}^{n}(-1)^{i} X_{i} \cdot f\left(X_{0}, \ldots, \hat{i}, \ldots, X_{n}\right)+ \\
& +\sum_{i<j}(-1)^{i+j} f\left(\left[X_{i}, X_{j}\right], X_{0}, \ldots, \hat{i}, \ldots, \hat{j}, \ldots, X_{n}\right),
\end{aligned}
$$

where the hats denote omission. Clearly, if we start with a representation $\mathbb{V}$ of the group $G$, then $\partial$ is a homomorphism of $G_{0}$-modules, and it is well known that $\partial \circ \partial=0$.

The crucial fact for us is that on this standard complex there is a Hodge theory, which was first introduced for complex simple Lie algebras in [25]. The most conceptual way to describe this Hodge structure is to use the natural duality between $\mathfrak{g}_{-}$and $\mathfrak{p}_{+}$via the Killing form. This is a duality of $G_{0}$-modules, but if we consider $\mathfrak{g}_{-}$as a $P$-module via the adjoint action and the identification with $\mathfrak{g} / \mathfrak{p}$, then it even is a duality of $P$ modules by invariance of the Killing form. Thus, given a representation $\mathbb{V}$ of $\mathfrak{g}$ and its dual $\mathbb{V}^{*}$, we can naturally identify $C^{n}\left(\mathfrak{p}_{+}, \mathbb{V}^{*}\right)$ with the dual 
$P$-module of $C^{n}\left(\mathfrak{g}_{-}, \mathbb{V}\right)$. Thus, the dual map to the Lie algebra differential $\partial: C^{n}\left(\mathfrak{p}_{+}, \mathbb{V}^{*}\right) \rightarrow C^{n+1}\left(\mathfrak{p}_{+}, \mathbb{V}^{*}\right)$ can be viewed as a linear map

$$
\partial^{*}: C^{n+1}\left(\mathfrak{g}_{-}, \mathbb{V}\right) \rightarrow C^{n}\left(\mathfrak{g}_{-}, \mathbb{V}\right)
$$

which is called the codifferential. From the above, it is obvious that the codifferential is a $G_{0}$-homomorphism and $\partial^{*} \circ \partial^{*}=0$. Moreover, one immediately verifies that the Lie algebra differential for $\mathfrak{p}_{+}$is even a $P$-homomorphism and thus the same is true for $\partial^{*}$.

A formula for $\partial^{*}$ can be easily computed for elements of the form $Z_{0} \wedge$ $\cdots \wedge Z_{n} \otimes v$, where the $Z_{i}$ are in $\mathfrak{p}_{+}$and $v$ is in $\mathbb{V}$. Pairing this element with a multilinear map $\psi \in C^{n+1}\left(\mathfrak{p}_{+}, \mathbb{V}^{*}\right)$, we simply get $\psi\left(Z_{0}, \ldots, Z_{n}\right)(v)$. Using this, one immediately computes that

$$
\begin{aligned}
\partial^{*}\left(Z_{0} \wedge \cdots \wedge Z_{n} \otimes v\right) & =\sum_{i=0}^{n}(-1)^{i+1} Z_{0} \wedge \cdots \hat{i} \cdots \wedge Z_{n} \otimes Z_{i} \cdot v+ \\
& +\sum_{i<j}(-1)^{i+j}\left[Z_{i}, Z_{j}\right] \wedge \cdots \hat{i} \cdots \hat{j} \cdots \wedge Z_{n} \otimes v .
\end{aligned}
$$

From this formula, it is again obvious that $\partial^{*}$ is a $P$-homomorphism.

Using Lie theory, one constructs an inner product on the spaces of cochains, with respect to which $\partial$ and $\partial^{*}$ are adjoint operators. The proof for this fact in the generality we need it is only a rather simple extension of results available in the literature, see e.g. [34, 35]. For the sake of completeness and the convenience of the reader, we give a complete proof in Appendix Appendix $\mathrm{B}$.

4.2. This adjointness result has a number of important consequences: First of all one gets a harmonic theory for the cohomology $H^{*}\left(\mathfrak{g}_{-}, \mathbb{V}\right)$. We define the Laplacian

$$
\square=\partial \circ \partial^{*}+\partial^{*} \circ \partial .
$$

Then for each $n$ this is a $G_{0}$-endomorphism of $C^{n}\left(\mathfrak{g}_{-}, \mathbb{V}\right)$. Moreover, the adjointness implies that $\operatorname{ker}(\square)=\operatorname{ker}(\partial) \cap \operatorname{ker}\left(\partial^{*}\right)$ and we have a $G_{0}$-invariant splitting

$$
C^{n}\left(\mathfrak{g}_{-}, V\right)=\operatorname{im}(\partial) \oplus \operatorname{ker}(\square) \oplus \operatorname{im}\left(\partial^{*}\right) .
$$

This implies then that the cohomology group $H^{n}\left(\mathfrak{g}_{-}, \mathbb{V}\right.$ ) is isomorphic (as a $G_{0}$-module) to the subspace $\operatorname{ker}(\square) \subset C^{n}\left(\mathfrak{g}_{-}, \mathbb{V}\right)$. Moreover, the situation between $\partial$ and $\partial^{*}$ is completely symmetric, so we can as well compute the cohomology groups $H^{*}\left(\mathfrak{g}_{-}, \mathbb{V}\right)$ as $\operatorname{ker}\left(\partial^{*}\right) / \operatorname{im}\left(\partial^{*}\right)$. This is more suitable for our purposes, since, as we have noticed above, $\partial^{*}$ is even a $P$-homomorphism. This also implies that (even as a $G_{0}$-module) the cohomology group $H^{n}\left(\mathfrak{g}_{-}, \mathbb{V}\right)$ is dual to $H^{n}\left(\mathfrak{p}_{+}, \mathbb{V}^{*}\right)$.

Thus, we get a canonical action of $P$ on the cohomology groups $H^{n}\left(\mathfrak{g}_{-}, \mathbb{V}\right)$. We claim, that this module is completely reducible, i.e. a direct sum of irreducibles. To prove this, we only have to show that $\mathfrak{p}_{+}$acts trivially on the cohomology groups. Fortunately, there is the following simple observation

Lemma. Let $Z \in \mathfrak{p}_{+}$and $f \in C^{n}\left(\mathfrak{g}_{-}, \mathbb{V}\right) \cong \Lambda^{n_{p_{+}}} \otimes \mathbb{V}$. Consider $Z \cdot f \in$ $C^{n}\left(\mathfrak{g}_{-}, \mathbb{V}\right)$ and $Z \wedge f \in C^{n+1}\left(\mathfrak{g}_{-}, \mathbb{V}\right)$, as defined in 3.9. Then

$$
\partial^{*}(Z \wedge f)=-Z \cdot f-Z \wedge \partial^{*}(f)
$$


Proof. This is a direct consequence of the general formula for $\partial^{*}$ in 4.1 .

Now, one immediately concludes that the $\mathfrak{p}_{+}-$action maps $\operatorname{ker}\left(\partial^{*}\right)$ to $\operatorname{im}\left(\partial^{*}\right)$, and thus in particular the induced action on the cohomology groups is trivial.

In [25], B. Kostant computed the cohomology groups $H^{*}\left(\mathfrak{p}_{+}, \mathbb{V}\right)$ in the case when $\mathfrak{g}$ is complex and simple and $\mathbb{V}$ is a complex irreducible representation. The basic idea in the proof is to analyze the action of the Laplacian $\square$ in terms of Casimir operators.

In fact, our construction of the sequences of natural operators will not need the explicit knowledge of the cohomologies. On the other hand, the full power of Kostant's theorem is necessary in order to compare the resulting sequences with the standard BGG-resolutions in representation theory.

Let us also remark here, that the knowledge of the second cohomologies with values in $\mathfrak{g}$ determines nicely the structure of the curvature of normal parabolic geometries, see e.g. [35, 30].

4.3. A sketch of the construction. Let us return to the twisted de Rham sequence

$$
\Gamma(V M) \rightarrow \Omega^{1}(M ; V M) \rightarrow \cdots \rightarrow \Omega^{\max }(M ; V M) \rightarrow 0
$$

on a manifold $M$ equipped with a parabolic geometry $(\mathcal{G}, \omega)$. For each $i$, $\Omega^{i}(M ; V M)$ is the space of sections (smooth in the real case, holomorphic in the complex setting) of the natural bundle associated to the representation $\Lambda^{i} \mathfrak{p}_{+} \otimes \mathbb{V}$. The maps $\partial, \partial^{*}$, and $\square$ defined above induce maps on the spaces of sections, which we denote by the same symbols. Moreover, since these maps are induced by pointwise operations the Hodge decomposition of $\Lambda^{i} \mathfrak{p}_{+} \otimes \mathbb{V}$ gives rise to a Hodge decomposition

$$
\Omega^{i}(M ; V M)=\operatorname{im}(\partial) \oplus \operatorname{ker}(\square) \oplus \operatorname{im}\left(\partial^{*}\right) .
$$

One has to be careful, however, that this decomposition is not $P$-invariant but just $G_{0}$-invariant, since $\partial^{*}$ is a $P$-homomorphism but $\partial$ and $\square$ are not. Thus the latter decomposition makes explicit geometrical sense only after a reduction of $\mathcal{G}$ to $G_{0}$, cf. the discussion in 2.13.

Since $\partial^{*}$ is a $P$-homomorphism, the kernel $\operatorname{ker}\left(\partial^{*}\right)$ and the image $\operatorname{im}\left(\partial^{*}\right)$ are the spaces of sections of natural subbundles of $\Lambda^{n} T^{*} M \otimes V M$. Moreover, from 4.2 we know that the quotient $\operatorname{ker}\left(\partial^{*}\right) / \operatorname{im}\left(\partial^{*}\right)$ can be identified with the space of sections of the bundle associated to the (completely reducible) representation $\mathbb{H}_{\mathbb{V}}^{n}=H^{n}\left(\mathfrak{g}_{-}, \mathbb{V}\right)$ of $P$, so we get an algebraic natural operator from the subset $\operatorname{ker}\left(\partial^{*}\right)$ of $\Omega^{n}(M ; V M)$ to the space of smooth sections of the natural bundle corresponding to the representation $\mathbb{H}_{\mathbb{V}}^{n}$. If $\mathbb{E}$ is an irreducible component of $\mathbb{H}_{\mathbb{V}}^{n}$, then we can further project onto this component to get an algebraic natural operator $\operatorname{ker}\left(\partial^{*}\right) \rightarrow \Gamma(E M)$.

On the other hand, $\mathbb{H}_{\mathbb{V}}^{n}$ can be identified with $\operatorname{ker}(\square) \subset \Lambda^{n} \mathfrak{p}_{+} \otimes \mathbb{V}$ as a $G_{0}$-module, so we may view any section of the corresponding bundle as a $V M$-valued $n$-form, but this is not a natural operator. The main point of the following will be that one can construct a natural differential operator $L$ from sections of the bundle corresponding to $\mathbb{H}_{\mathbb{V}}^{n}$ to $V M$-valued $n$-forms in $\operatorname{ker}\left(\partial^{*}\right)$, which has this inclusion as the lowest homogeneous component. Otherwise put, one can split the algebraic projection $\pi$ constructed above by a natural differential operator $L$. Moreover, it will turn out that this 
operator is fully determined by the following surprising fact: For each section $\alpha \in \Gamma\left(H_{\mathbb{V}}^{n} M\right)$ there exists the unique section $L(\alpha) \in \operatorname{ker}\left(\partial^{*}\right) \subset \Omega^{n}(M ; V M)$ such that $\pi \circ L(\alpha)=\alpha$ and $d_{\mathbb{V}}(L(\alpha)) \in \operatorname{ker}\left(\partial^{*}\right) \subset \Omega^{n+1}(M ; V M)$.

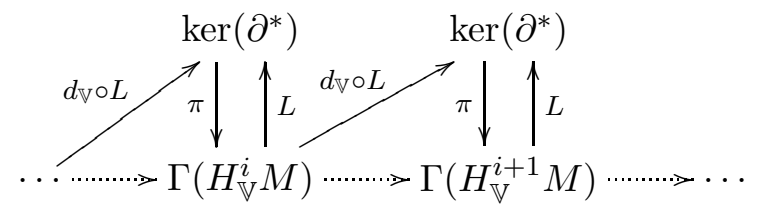

Summarizing the prospective achievement, the twisted exterior derivatives will produce plenty of natural differential operators indicated by the dotted arrows in the diagram.

The idea for the construction of this natural differential operator $L$ is fairly simple. Recall from 3.9 that the lowest homogeneous component of $d_{\mathbb{V}}$ equals the Lie algebra differential $\partial$. Suppose we have a section $s$ in the bundle corresponding to $\mathbb{H}_{\mathbb{V}}^{n}$, which is homogeneous of some degree $i$. Then it lies in $\operatorname{ker}(\square)$ and thus in particular in $\operatorname{ker}(\partial)$, so the homogeneous component of degree $i$ of $d_{\mathbb{V}}(s)$ is automatically zero. The idea is now to extend $s$ to $\tilde{s}$ in such a way that $d_{\mathbb{V}}(\tilde{s})$ is as small as possible. The homogeneous component of degree $i+1$ of $d_{\mathbb{V}}(s)$ can be split into components in $\operatorname{im}(\partial), \operatorname{ker}(\square)$, and $\operatorname{im}\left(\partial^{*}\right)$, and the best we can do to kill it is to add to $s$ an element $s_{i+1}$ which is homogeneous of degree $i+1$ such that $\partial\left(s_{i+1}\right)$ is the negative of the $\operatorname{im}(\partial)-$ component of $d_{\mathbb{V}}(s)$ in degree $i+1$. There is a freedom in the choice of $s_{i+1}$ which can be fixed by requiring that $s_{i+1} \in \operatorname{im}\left(\partial^{*}\right)$ (which is a complement to $\operatorname{ker}(\partial)$ by the adjointness). But this allows us already to compute $s_{i+1}$ : Since $\partial^{*}\left(s_{i+1}\right)=0$ we see that $\square\left(s_{i+1}\right)=\partial^{*}\left(\partial\left(s_{i+1}\right)\right)$. But $\partial\left(s_{i+1}\right)$ is just the negative of the $\operatorname{im}(\partial)$-part of the homogeneous component of degree $i+1$ of $d_{\mathbb{V}}(s)$, so this is known. Moreover, by definition $\square$ commutes both with $\partial$ and $\partial^{*}$, and $\operatorname{ker}(\square) \cap \operatorname{im}\left(\partial^{*}\right)=\{0\}$. Thus $\square$ restricts to an isomorphism $\operatorname{im}\left(\partial^{*}\right) \rightarrow \operatorname{im}\left(\partial^{*}\right)$. Hence we can compute $s_{i+1}$ by applying $\square^{-1} \circ \partial^{*}$ to the homogeneous component of degree $i+1$ of $d_{\mathbb{V}}(s)$. Similarly we can continue to add an appropriate homogeneous component of degree $i+2$ and so on.

From this description it is not at all obvious that this construction produces a natural operator, since the map $\square^{-1}$ involved in the construction is not a $P$-homomorphism, and the subsequent steps of the construction use $d_{\mathbb{V}}-\partial$ which is not natural either. Below we will manage, however, to work out the procedure sketched above within the framework of homomorphisms between semi-holonomic jet modules. Thus the resulting operators $L$ will be even strongly invariant.

\subsection{Each $P$-module $\mathbb{V}$ enjoys a decomposition}

$$
\mathbb{V}=\mathbb{V}_{i_{0}} \oplus \mathbb{V}_{i_{0}+1} \oplus \cdots \oplus \mathbb{V}_{i_{0}+k}
$$

as a $G_{0}$-module, where the submodules $\mathbb{V}_{i}$ are distinguished by the requirement that the grading element $E \in \mathfrak{g}_{0}$ (cf. 2.1) acts by scalar multiplication by $i$. The action of the elements $Z \in \mathfrak{g}_{j}$ then maps $\mathbb{V}_{i}$ into $\mathbb{V}_{i+j}$ and so for each $j=0, \ldots, k$ the subspace $\mathbb{V}^{j}:=\mathbb{V}_{i_{0}+j} \oplus \cdots \oplus \mathbb{V}_{i_{0}+k}$ is a $P$-submodule of $\mathbb{V}$. In particular, this decomposition of an irreducible $G$-module $\mathbb{V}$, viewed as $P$-module, runs from $\mathbb{V}_{-k}$ to $\mathbb{V}_{k}$, where $\mathbb{V}_{k}$ is the $P$-submodule generated by the highest weight of $\mathbb{V}$. 
Now, let $\mathbb{E}_{i_{0}}$ be an irreducible component of $H^{n}\left(\mathfrak{g}_{-}, \mathbb{V}\right)$, on which the grading element acts by multiplication by $i_{0}$. Then we can view $\mathbb{E}_{i_{0}}$ as a $G_{0}$ submodule of $\operatorname{ker}(\square) \subset \Lambda^{n} \mathfrak{p}_{+} \otimes \mathbb{V}$ and we write $\mathbb{E}$ for the $P$-submodule in $\Lambda^{n} \mathfrak{p}_{+} \otimes \mathbb{V}$ generated by $\mathbb{E}_{i_{0}}$. Let

$$
\mathbb{E}=\mathbb{E}_{i_{0}} \oplus \cdots \oplus \mathbb{E}_{i_{0}+r}
$$

be the above $G_{0}$-module decomposition according to eigenvalues of the grading element. Then the action of $\mathfrak{g}_{\ell}$ maps each $\mathbb{E}_{i_{0}+i}$ to $\mathbb{E}_{i_{0}+i+\ell}$. For each $i=1, \ldots, r+1$ we have the $P$-submodule $\mathbb{E}^{i}$ as above, so we can form the quotient $\mathbb{E} / \mathbb{E}^{i}$, which is as a $G_{0}$-module isomorphic to $\mathbb{E}_{i_{0}} \oplus \cdots \oplus \mathbb{E}_{i_{0}+i-1}$. In particular, $\mathbb{E} / \mathbb{E}^{1}$ is again the irreducible module $\mathbb{E}_{i_{0}}$ we started with but now viewed as a $P$-module, and $\mathbb{E} / \mathbb{E}^{r+1}=\mathbb{E}$.

Lemma. (1) $\mathbb{E} \subset \operatorname{ker}\left(\partial^{*}\right)$ and $\mathbb{E}^{1} \subset \operatorname{im}\left(\partial^{*}\right)$.

(2) The Laplacian $\square$ restricts to a $G_{0}$-isomorphism $\mathbb{E}_{i_{0}+i} \rightarrow \mathbb{E}_{i_{0}+i}$ for each $i=1, \ldots, r$.

Proof. (1) The first part is clear, since $\operatorname{ker}\left(\partial^{*}\right)$ is a $P$-submodule which by construction contains $\mathbb{E}_{i_{0}}$. Since we have already seen in Lemma 4.2 that the action of $\mathfrak{p}_{+}$maps $\operatorname{ker}\left(\partial^{*}\right)$ to $\operatorname{im}\left(\partial^{*}\right)$, the second part is also clear.

(2) We have already noted in 4.3 above that $\square$ restricts to an automorphism on $\operatorname{im}\left(\partial^{*}\right)$. Hence it suffices to prove that $\square\left(\mathbb{E}_{i_{0}+i}\right) \subset \mathbb{E}_{i_{0}+i}$. By Corollary 3.10, we have for all $e \in \mathbb{E}, Z \in \mathfrak{g}_{1}$

$$
\partial(Z \cdot e)=Z \cdot \partial(e)-(n+1) \sum_{\left|\eta_{\alpha}\right|=1} \eta_{\alpha} \wedge\left[Z, \xi_{\alpha}\right] \cdot e .
$$

Applying $\partial^{*}$ to the first term we get $Z \cdot \square(e)$.

Let us first take $e_{0} \in \mathbb{E}_{i_{0}}$, and consider $\square\left(Z \cdot e_{0}\right)=\partial^{*}\left(\partial\left(Z \cdot e_{0}\right)\right)$. Then the first term vanishes while each summand in the second term is contained in $\partial^{*}\left(\mathfrak{g}_{1} \wedge \mathfrak{g}_{0} \cdot \mathbb{E}_{i_{0}}\right) \subset \partial^{*}\left(\mathfrak{g}_{1} \wedge \mathbb{E}_{i_{0}}\right)$. Since $\mathbb{E}_{i_{0}} \subset \operatorname{ker}\left(\partial^{*}\right)$, Lemma 4.2 implies that $\partial^{*}\left(\mathfrak{g}_{1} \wedge \mathbb{E}_{i_{0}}\right) \subset \mathfrak{g}_{1} \cdot \mathbb{E}_{i_{0}} \subset \mathbb{E}_{i_{0}+1}$. Thus, we see that $\square\left(\mathbb{E}_{i_{0}+1}\right) \subset \mathbb{E}_{i_{0}+1}$. Now one can proceed inductively in the same way to show that $\square\left(\mathbb{E}_{i_{0}+i}\right) \subset \mathbb{E}_{i_{0}+i}$.

4.5. The actual construction of the splitting operators is a little tricky. The problem is that the individual steps in the construction sketched in 4.3 are induced by maps on jet-modules which are not $P$-module homomorphisms but only restrict to $P$-module homomorphisms on appropriate submodules, which also have to be constructed during the procedure.

For $j \geq i \geq 0$ we denote by $\pi_{i}^{j}$ the canonical projection $\mathbb{E} / \mathbb{E}^{j} \rightarrow \mathbb{E} / \mathbb{E}^{i}$, which is a homomorphism of $P$-modules. Clearly, $\pi_{i}^{i}$ is the identity and $\pi_{i}^{j} \circ \pi_{j}^{k}=\pi_{i}^{k}$ for $i \leq j \leq k$. By $p_{i}: \mathcal{J}^{1}\left(\mathbb{E} / \mathbb{E}^{i}\right) \rightarrow \mathbb{E} / \mathbb{E}^{i}$ we denote the footpoint projection, which is a $P$-homomorphism, too. For any element $\psi$ in a general $G_{0}$-module, we denote by $\psi_{i}$ the component of $\psi$ on which the grading element $E$ acts by multiplication by $i_{0}+i$. Note that the mapping $\psi \mapsto \psi_{i}$ is only a $G_{0}$-homomorphism and not a $P$-homomorphism. Finally, let us denote by $j_{i}: \mathbb{E} / \mathbb{E}^{i} \rightarrow \mathbb{E} / \mathbb{E}^{i+1}$ the $G_{0}$-homomorphism given by the inclusion $\mathbb{E}_{i_{0}} \oplus \cdots \oplus \mathbb{E}_{i_{0}+i-1} \rightarrow \mathbb{E}_{i_{0}} \oplus \cdots \oplus \mathbb{E}_{i_{0}+i}$. Again, this is obviously not a $P$-homomorphism. Finally, let Alt $: \mathfrak{p}_{+} \otimes \Lambda^{n} \mathfrak{p}_{+} \otimes \mathbb{V} \rightarrow \Lambda^{n+1} \mathfrak{p}_{+} \otimes \mathbb{V}$ denote the alternation mapping. This is a $P$-homomorphism preserving homogeneous degrees. 
For $i=1, \ldots, r+1$ consider now the module $\mathcal{J}^{1}\left(\mathbb{E} / \mathbb{E}^{i}\right)$. A typical element of this module is a pair $(e, \psi)$, with $e \in \mathbb{E} / \mathbb{E}^{i}$ and

$$
\psi \in \mathfrak{p}_{+} \otimes \mathbb{E} / \mathbb{E}^{i} \subset \mathfrak{p}_{+} \otimes \Lambda^{n} \mathfrak{p}_{+} \otimes \mathbb{V}
$$

Now we define a mapping $L_{i}: \mathcal{J}^{1}\left(\mathbb{E} / \mathbb{E}^{i}\right) \rightarrow \mathbb{E} / \mathbb{E}^{i+1}$ by

$$
L_{i}(e, \psi)=j_{i}(e)-(n+1) \square^{-1} \partial^{*}\left((\operatorname{Alt}(\psi))_{i}\right) .
$$

In particular, if $\psi=Z \otimes f$ for $Z \in \mathfrak{p}_{+}$and $f \in \mathbb{E} / \mathbb{E}^{i}$, then $L_{i}(e, Z \otimes$ $f)=j_{i}(e)-(n+1) \square^{-1} \partial^{*}\left((Z \wedge f)_{i}\right)$. Now the main technical step in the construction is the following

4.6. Proposition. The maps $L_{i}: \mathcal{J}^{1}\left(\mathbb{E} / \mathbb{E}^{i}\right) \rightarrow \mathbb{E} / \mathbb{E}^{i+1}$ have the following properties:

(1) $L_{i}$ is a $G_{0}$-homomorphism and $\pi_{i}^{i+1} \circ L_{i}=p_{i}$.

(2) For $\Psi \in \mathcal{J}^{1}\left(\mathbb{E} / \mathbb{E}^{i}\right)$ and $W \in \mathfrak{g}_{1}$, we have

$$
L_{i}(W \cdot \Psi)-W \cdot L_{i}(\Psi)=\square^{-1}\left(W \cdot\left(\square \circ j_{i} \circ\left(L_{i-1} \circ \mathcal{J}^{1}\left(\pi_{i-1}^{i}\right)-p_{i}\right)(\Psi)\right)\right) .
$$

In particular, $L_{1}$ is a $P$-homomorphism.

Proof. (1) The fact that $L_{i}$ is a $G_{0}$-homomorphism follows immediately from the fact that $\mathcal{J}^{1}\left(\mathbb{E} / \mathbb{E}^{i}\right) \cong \mathbb{E} / \mathbb{E}^{i} \oplus\left(\mathfrak{p}_{+} \otimes \mathbb{E} / \mathbb{E}^{i}\right)$ as a $G_{0}$-module, see 3.2 and the definition of $L_{i}$. Moreover, since Alt, $\partial^{*}$, and $\square$ all preserve homogeneities, the last term in the definition of $L_{i}$ is homogeneous of degree $i_{0}+i$, so it lies in the kernel of $\pi_{i}^{i+1}$ and the second part follows.

(2) Clearly, it suffices to check this for elements $\Psi$ of the form $(e, Z \otimes f)$ with $e, f \in \mathbb{E} / \mathbb{E}^{i}$ and $Z \in \mathfrak{p}_{+}$. By definition of the action on jets, see 3.2, we see that $W \cdot(e, Z \otimes f)$ has footpoint $W \cdot e$, while the homogeneous part of degree $i_{0}+i$ of the second component is given by

$$
\sum_{\left|\eta_{\alpha}\right|=1} \eta_{\alpha} \otimes\left[W, \xi_{\alpha}\right] \cdot e_{i-1}+W \cdot(Z \otimes f)_{i-1}
$$

Consequently,

$$
\begin{aligned}
L_{i}(W \cdot(e, Z \otimes f))= & j_{i}(W \cdot e)-(n+1) \square^{-1} \partial^{*}\left(\sum_{\left|\eta_{\alpha}\right|=1} \eta_{\alpha} \wedge\left[W, \xi_{\alpha}\right] \cdot e_{i-1}\right)- \\
& -(n+1) \square^{-1} \partial^{*}\left(W \cdot(Z \wedge f)_{i-1}\right) .
\end{aligned}
$$

By Corollary 3.10 the second term on the right hand side of this equation just gives

$$
\square^{-1} \partial^{*}\left(\partial\left(W \cdot e_{i-1}\right)-W \cdot \partial\left(e_{i-1}\right)\right)=W \cdot e_{i-1}-\square^{-1}\left(W \cdot \square\left(e_{i-1}\right)\right),
$$

where we have used that $\partial^{*}$ is a $P$-homomorphism, $e_{i-1}$ and $W \cdot e_{i-1}$ lie in the kernel of $\partial^{*}$, and that we are in a region where the Laplacian is invertible. On the other hand, we clearly have $j_{i}(W \cdot e)+W \cdot e_{i-1}=W \cdot j_{i}(e)$, since $W \in \mathfrak{g}_{1}$ and $e_{i-1}$ is the highest nonzero homogeneous component of $e$. Finally, we clearly have $W \cdot L_{i}(e, Z \otimes f)=W \cdot j_{i}(e)$, since the rest lies in the component of maximal homogeneity, on which $\mathfrak{p}_{+}$acts trivially. Thus, we have arrived at

$$
\begin{aligned}
L_{i}(W \cdot(e, Z \otimes f) & -W \cdot L_{i}(e, Z \otimes f)= \\
= & -\square^{-1}\left(W \cdot \square\left(e_{i-1}\right)\right)-(n+1) \square^{-1}\left(W \cdot \partial^{*}\left((Z \wedge f)_{i-1}\right)\right),
\end{aligned}
$$


where we have used once more the fact that $\partial^{*}$ is a $P$-homomorphism.

On the other hand, consider $\mathcal{J}^{1}\left(\pi_{i-1}^{i}\right)(e, Z \otimes f)$. The footpoint of this element is just $\pi_{i-1}^{i}(e)$, while in the jet part, the component of maximal homogeneity must coincide with $(Z \otimes f)_{i-1}$. Consequently, we get

$$
L_{i-1}\left(\mathcal{J}^{1}\left(\pi_{i-1}^{i}\right)(e, Z \otimes f)\right)=j_{i-1}\left(\pi_{i-1}^{i}(e)\right)-(n+1) \square^{-1} \partial^{*}\left((Z \wedge f)_{i-1}\right) .
$$

Subtracting $e=p_{i}(e, Z \otimes f)$ from this, we get

$$
-e_{i-1}-(n+1) \square^{-1} \partial^{*}\left((Z \wedge f)_{i-1}\right),
$$

and the formula follows. In the case $i=1$, we get $L_{1}(W \cdot \Psi)-W \cdot L_{1}(\Psi)=$ $-\square^{-1}\left(W \cdot\left(\square \circ j_{1} \circ p_{1}\right)(\Psi)\right)$, which vanishes, since $\mathbb{E}_{i_{0}} \subset \operatorname{Ker}(\square)$. Hence, $L_{1}$ is equivariant for the action of $\mathfrak{g}_{1}$ and thus a $P$-homomorphism by (1) and 2.4 .

4.7. Now we inductively define subspaces $\tilde{\mathcal{J}}^{1}\left(\mathbb{E} / \mathbb{E}^{i}\right) \subset \mathcal{J}^{1}\left(\mathbb{E} / \mathbb{E}^{i}\right)$ by $\tilde{\mathcal{J}}^{1}\left(\mathbb{E} / \mathbb{E}^{1}\right)=$ $\mathcal{J}^{1}\left(\mathbb{E} / \mathbb{E}^{1}\right)$ and

$$
\tilde{\mathcal{J}}^{1}\left(\mathbb{E} / \mathbb{E}^{i+1}\right)=\mathcal{J}^{1}\left(\pi_{i}^{i+1}\right)^{-1}\left(\tilde{\mathcal{J}}^{1}\left(\mathbb{E} / \mathbb{E}^{i}\right)\right) \cap \operatorname{Ker}\left(L_{i} \circ \mathcal{J}^{1}\left(\pi_{i}^{i+1}\right)-p_{i+1}\right) .
$$

Proposition. For each $i=1, \ldots, r+1$ the space $\tilde{\mathcal{J}}^{1}\left(\mathbb{E} / \mathbb{E}^{i}\right) \subset \mathcal{J}^{1}\left(\mathbb{E} / \mathbb{E}^{i}\right)$ is a $P$-submodule and $L_{i}$ restricts to a homomorphism $\tilde{\mathcal{J}}^{1}\left(\mathbb{E} / \mathbb{E}^{i}\right) \rightarrow \mathbb{E} / \mathbb{E}^{i+1}$ of $P$-modules. Moreover, for each $k<i$ we have

$$
\mathcal{J}^{1}\left(\pi_{k}^{i}\right)\left(\tilde{\mathcal{J}}^{1}\left(\mathbb{E} / \mathbb{E}^{i}\right)\right) \subset \tilde{\mathcal{J}}^{1}\left(\mathbb{E} / \mathbb{E}^{k}\right),
$$

and on $\tilde{\mathcal{J}}^{1}\left(\mathbb{E} / \mathbb{E}^{i}\right)$ we have $\pi_{k+1}^{i} \circ p_{i}=L_{k} \circ \mathcal{J}^{1}\left(\pi_{k}^{i}\right)$.

Proof. For $i=1$ the first two properties are satisfied by definition of $\tilde{\mathcal{J}}^{1}\left(\mathbb{E} / \mathbb{E}^{1}\right)$ and Proposition 4.6(2), while the last two properties are trivially satisfied. If we inductively assume that the result has been proved for $i-1$, then $\mathcal{J}^{1}\left(\pi_{i-1}^{i}\right)^{-1}\left(\tilde{\mathcal{J}}^{1}\left(\mathbb{E} / \mathbb{E}^{i-1}\right)\right)$ is a $P$-submodule of $\mathcal{J}^{1}\left(\mathbb{E} / \mathbb{E}^{i}\right)$, and $L_{i-1}$ 。 $\mathcal{J}^{1}\left(\pi_{i-1}^{i}\right)-p_{i}$ defines a $P$-homomorphism from this submodule to $\mathbb{E} / \mathbb{E}^{i}$, so $\tilde{\mathcal{J}}^{1}\left(\mathbb{E} / \mathbb{E}^{i}\right)$ is a $P$-submodule. Moreover, Proposition $4.6(2)$ immediately implies that the restriction of $L_{i}$ to this submodule is equivariant under the action of $\mathfrak{g}_{1}$ and thus $L_{i}$ restricts to a $P$-homomorphism on that submodule by Proposition 4.6(1) and 2.4. Moreover, we get the last two properties in the case $k=i-1$.

For $k<i-1$, note first that $\pi_{k}^{i}=\pi_{k}^{i-1} \circ \pi_{i-1}^{i}$ immediately implies that $\mathcal{J}^{1}\left(\pi_{k}^{i}\right)\left(\tilde{\mathcal{J}}^{1}\left(\mathbb{E} / \mathbb{E}^{i}\right)\right) \subset \tilde{\mathcal{J}}^{1}\left(\mathbb{E} / \mathbb{E}^{k}\right)$ by induction. Finally, we compute

$$
\begin{aligned}
L_{k} \circ \mathcal{J}^{1}\left(\pi_{k}^{i}\right) & =L_{k} \circ \mathcal{J}^{1}\left(\pi_{k}^{i-1}\right) \circ \mathcal{J}^{1}\left(\pi_{i-1}^{i}\right)=\pi_{k+1}^{i-1} \circ p_{i-1} \circ \mathcal{J}^{1}\left(\pi_{i-1}^{i}\right)= \\
& =\pi_{k+1}^{i-1} \circ \pi_{i-1}^{i} \circ p_{i}=\pi_{k+1}^{i} \circ p_{i},
\end{aligned}
$$

by functoriality of $\mathcal{J}^{1}$, induction, and the definition of the jet prolongation of a homomorphism.

For $k \geq 2$ and $i=1, \ldots, r+1$ we inductively define

$$
\tilde{\mathcal{J}}^{k}\left(\mathbb{E} / \mathbb{E}^{i}\right):=\mathcal{J}^{1}\left(\tilde{\mathcal{J}}^{k-1}\left(\mathbb{E} / \mathbb{E}^{i}\right)\right) \cap \overline{\mathcal{J}}^{k}\left(\mathbb{E} / \mathbb{E}^{i}\right) .
$$

By Proposition 4.7 and 3.4 it follows inductively that $\tilde{\mathcal{J}}^{k}\left(\mathbb{E} / \mathbb{E}^{i}\right)$ is a $P_{-}$ submodule in both modules on the right hand side of the definition. For $i=$ 1 , we obtain $\tilde{\mathcal{J}}^{k}\left(\mathbb{E} / \mathbb{E}^{1}\right)=\overline{\mathcal{J}}^{k}\left(\mathbb{E} / \mathbb{E}^{1}\right)$, so we simply get the full semiholonomic 
jet-module in this case. Moreover, a simple inductive argument shows for all $\ell<k$, and $i$

$$
\tilde{\mathcal{J}}^{k}\left(\mathbb{E} / \mathbb{E}^{i}\right) \subset \overline{\mathcal{J}}^{\ell}\left(\tilde{\mathcal{J}}^{k-\ell}\left(\mathbb{E} / \mathbb{E}^{i}\right)\right) \cap \overline{\mathcal{J}}^{k}\left(\mathbb{E} / \mathbb{E}^{i}\right) .
$$

For each of the homomorphisms $L_{i}: \tilde{\mathcal{J}}^{1}\left(\mathbb{E} / \mathbb{E}^{i}\right) \rightarrow \mathbb{E} / \mathbb{E}^{i+1}$ we can now restrict the semiholonomic jet prolongation $\overline{\mathcal{J}}^{k}\left(L_{i}\right)$ to the submodule $\tilde{\mathcal{J}}^{k+1}\left(\mathbb{E} / \mathbb{E}^{i}\right) \subset$ $\overline{\mathcal{J}}^{k}\left(\tilde{\mathcal{J}}^{1}\left(\mathbb{E} / \mathbb{E}^{i}\right)\right)$ to obtain a $P$-homomorphism

$$
\overline{\mathcal{J}}^{k}\left(L_{i}\right): \tilde{\mathcal{J}}^{k+1}\left(\mathbb{E} / \mathbb{E}^{i}\right) \rightarrow \overline{\mathcal{J}}^{k}\left(\mathbb{E} / \mathbb{E}^{i+1}\right) .
$$

4.8. Theorem. Let $\mathbb{E}_{i_{0}}$ be an irreducible component in the cohomology $\mathbb{H}_{\mathbb{V}}^{n}$

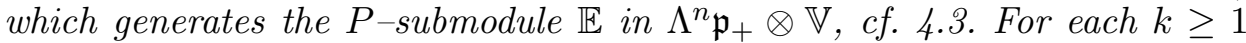
and $i=1, \ldots, r+1$ we have

$$
\overline{\mathcal{J}}^{k}\left(L_{i}\right)\left(\tilde{\mathcal{J}}^{k+1}\left(\mathbb{E} / \mathbb{E}^{i}\right)\right) \subset \tilde{\mathcal{J}}^{k}\left(\mathbb{E} / \mathbb{E}^{i+1}\right) .
$$

In particular, the composition

$$
L:=L_{r} \circ \overline{\mathcal{J}}^{1}\left(L_{r-1}\right) \circ \ldots \circ \overline{\mathcal{J}}^{r-1}\left(L_{1}\right)
$$

defines a P-homomorphism $L: \overline{\mathcal{J}}^{r}\left(\mathbb{E} / \mathbb{E}_{1}\right) \rightarrow \mathbb{E}$. Since by definition $\mathbb{E}$ is a $P$-submodule of $\Lambda^{n} \mathfrak{p}_{+} \otimes \mathbb{V}$, this homomorphism induces a strongly invariant operator $\Gamma\left(E_{i_{0}} M\right) \rightarrow \operatorname{Ker}\left(\partial^{*}\right) \subset \Omega^{n}(M ; V M)$, which splits the algebraic projection $\operatorname{Ker}\left(\partial^{*}\right) \rightarrow \Gamma\left(E_{i_{0}} M\right)$ described in 4.3 .

Proof. Let us first consider the case $k=1$. So we have to show that $\mathcal{J}^{1}\left(L_{i}\right)\left(\tilde{\mathcal{J}}^{2}\left(\mathbb{E} / \mathbb{E}^{i}\right)\right) \subset \tilde{\mathcal{J}}^{1}\left(\mathbb{E} / \mathbb{E}^{i+1}\right)$. By definition of $\tilde{\mathcal{J}}^{1}\left(\mathbb{E} / \mathbb{E}^{i+1}\right)$, we first have to consider the composition $\mathcal{J}^{1}\left(\pi_{i}^{i+1}\right) \circ \mathcal{J}^{1}\left(L_{i}\right)=\mathcal{J}^{1}\left(\pi_{i}^{i+1} \circ L_{i}\right)$. By Proposition 4.6 (1), this equals $\mathcal{J}^{1}\left(p_{i}\right)$. Since $\tilde{\mathcal{J}}^{2}\left(\mathbb{E} / \mathbb{E}^{i}\right) \subset \overline{\mathcal{J}}^{2}\left(\mathbb{E} / \mathbb{E}^{i}\right)$, this projection coincides with the restriction of the canonical projection $\overline{\mathcal{J}}^{2}\left(\mathbb{E} / \mathbb{E}^{i}\right) \rightarrow$ $\mathcal{J}^{1}\left(\mathbb{E} / \mathbb{E}^{i}\right)$, and since $\tilde{\mathcal{J}}^{2}\left(\mathbb{E} / \mathbb{E}^{i}\right) \subset \mathcal{J}^{1}\left(\tilde{\mathcal{J}}^{1}\left(\mathbb{E} / \mathbb{E}^{i}\right)\right)$, this canonical projection has values in $\tilde{\mathcal{J}}^{1}\left(\mathbb{E} / \mathbb{E}^{i}\right)$. Thus, we have verified that $\mathcal{J}^{1}\left(L_{i}\right)\left(\tilde{\mathcal{J}}^{2}\left(\mathbb{E} / \mathbb{E}^{i}\right)\right) \subset$ $\mathcal{J}^{1}\left(\pi_{i}^{i+1}\right)^{-1}\left(\tilde{\mathcal{J}}^{1}\left(\mathbb{E} / \mathbb{E}^{i}\right)\right)$.

But then it also follows that $L_{i} \circ \mathcal{J}^{1}\left(\pi_{i}^{i+1}\right) \circ \mathcal{J}^{1}\left(L_{i}\right)$ coincides with the composition of $L_{i}$ with the canonical projection $\tilde{\mathcal{J}}^{2}\left(\mathbb{E} / \mathbb{E}^{i}\right) \rightarrow \tilde{\mathcal{J}}^{1}\left(\mathbb{E} / \mathbb{E}^{i}\right)$, which by definition of the jet prolongation of a homomorphism (see 3.2) coincides with $p_{i+1} \circ \mathcal{J}^{1}\left(L_{i}\right)$ and the proof in the case $k=1$ is complete.

The case $k \geq 2$ now immediately follows from the definitions by induction. Thus, also the existence of $L$ and the corresponding strongly invariant operator is clear. The fact that this operator splits the algebraic projection follows from the fact that by Lemma 4.4.(1) this algebraic projection is induced by the canonical projection $\mathbb{E} \rightarrow \mathbb{E} / \mathbb{E}^{1}$ and the fact that $\pi_{i}^{i+1} \circ L_{i}=p_{i}$ from Proposition 4.6(1).

Next, we consider the composition of $d_{\mathbb{V}}$ with the operator corresponding to $L$. The corresponding homomorphism on jet modules can be computed as the restriction to $\overline{\mathcal{J}}^{r+1}\left(\mathbb{E} / \mathbb{E}^{1}\right)$ of $d_{\mathbb{V}} \circ \mathcal{J}^{1}(L)$.

4.9. Proposition. For each irreducible $G$-module $\mathbb{V}$, and irreducible $G_{0^{-}}$ submodule $\mathbb{E}_{i_{0}} \subset \mathbb{H}_{\mathbb{V}}^{n}=H^{n}\left(\mathfrak{g}_{-}, \mathbb{V}\right)$, the composition

$$
d_{\mathbb{V}} \circ \mathcal{J}^{1} L: \overline{\mathcal{J}}^{r+1}\left(\mathbb{E} / \mathbb{E}^{1}\right) \rightarrow \Lambda^{n+1} \mathfrak{p}_{+} \otimes \mathbb{V}
$$


has values in $\operatorname{ker} \partial^{*}$. The composition with the projection to the cohomology $\pi_{H}:\left(\Lambda^{n} \mathfrak{p}_{+} \otimes \mathbb{V}\right) \cap\left(\operatorname{ker} \partial^{*}\right) \rightarrow \mathbb{H}_{\mathbb{V}}^{n+1}=H^{n+1}\left(\mathfrak{g}_{-}, \mathbb{V}\right)$ gives the P-module homomorphism

$$
\pi_{H} \circ d_{\mathbb{V}} \circ \mathcal{J}^{1} L: \overline{\mathcal{J}}^{r+1}\left(\mathbb{E} / \mathbb{E}^{1}\right) \rightarrow \mathbb{H}_{\mathbb{V}}^{n+1} .
$$

For each $n=0, \ldots, \operatorname{dim} M-1$, there is the strongly invariant differential operator

$$
D^{\mathbb{V}}: \Gamma\left(H_{\mathbb{V}}^{n} M\right) \rightarrow \Gamma\left(H_{\mathbb{V}}^{n+1} M\right)
$$

whose restrictions to the subbundles $E_{0} M$ are determined by the above $P$ module homomorphisms $\overline{\mathcal{J}}^{r+1}\left(\mathbb{E} / \mathbb{E}^{1}\right) \rightarrow \mathbb{H}_{\mathbb{V}}^{n+1}$.

Proof. Consider first the map $\partial^{*} \circ d_{\mathbb{V}}: \mathcal{J}^{1}(\mathbb{E}) \rightarrow \Lambda^{n_{\mathfrak{p}}} \mathfrak{p}_{+} \otimes \mathbb{V}$. By definition of $d_{\mathbb{V}}$, Lemma 4.2, and using the fact that $\mathbb{E} \subset \operatorname{Ker}\left(\partial^{*}\right)$, we see that this maps $(e, Z \otimes f) \in \mathcal{J}^{1}(\mathbb{E})$ to $\partial^{*} \partial(e)+(n+1) \partial^{*}(Z \wedge f)=\square(e)-(n+1) Z \cdot f$, so $\partial^{*} \circ d_{\mathbb{V}}: \mathcal{J}^{1}(\mathbb{E}) \rightarrow \mathbb{E}$. Now Theorem 4.8 applied to $\mathcal{J}^{1}\left(L_{r}\right)$ shows, that $\mathcal{J}^{1}(L)$ has values in the submodule $\tilde{J}^{1}(\mathbb{E}) \subset \mathcal{J}^{1}(\mathbb{E})$, and we claim that $\partial^{*} \circ d_{\mathbb{V}}$ restricts to zero on that submodule.

To simplify notations, let us write $p: \mathcal{J}^{1}(\mathbb{E}) \rightarrow \mathbb{E}$ for the footpoint projection $p_{r+1}$ and $\pi_{i}$ for $\pi_{i}^{r+1}$. For $i \leq r+1$ consider the $P$-homomorphism $\pi_{i} \circ \partial^{*} \circ d_{\mathbb{V}}: \mathcal{J}^{1}(\mathbb{E}) \rightarrow \mathbb{E} / \mathbb{E}^{i}$. By definition, this maps $(e, Z \otimes f)$ to $\pi_{i}(\square(e))+$ $(n+1) \pi_{i}\left(\partial^{*}(Z \wedge f)\right)$. Since the Laplacian and $\partial^{*}$ both preserve homogeneous degrees, we may rewrite the first summand as $\square\left(\pi_{i}(e)\right)$ and the second summand as $(n+1) \pi_{i}\left(\partial^{*}\left(Z \wedge \pi_{i-1}(f)\right)\right)$.

On the other hand, consider $\mathcal{J}^{1}\left(\pi_{i-1}\right): \mathcal{J}^{1}(\mathbb{E}) \rightarrow \mathcal{J}^{1}\left(\mathbb{E} / \mathbb{E}^{i-1}\right)$. This maps $(e, Z \otimes f)$ to $\left(\pi_{i-1}(e), Z \otimes \pi_{i-1}(f)\right)$, and applying $L_{i-1}$ to this element, we get $j_{i-1}\left(\pi_{i-1}(e)\right)-(n+1) \square^{-1} \partial^{*}\left(\left(Z \wedge \pi_{i-1}(f)\right)_{i}\right)$. Finally, $\pi_{i} \circ p$ maps $(e, Z \otimes f)$ to $\pi_{i}(e)$. Consequently, $\square \circ\left(\pi_{i} \circ p-L_{i-1} \circ \mathcal{J}^{1}\left(\pi_{i-1}\right)\right)$ maps $(e, Z \otimes f)$ to

$$
\square\left(\pi_{i}(e)\right)-j_{i-1}\left(\square\left(\pi_{i-1}(e)\right)\right)+(n+1) \partial^{*}\left(\left(Z \wedge \pi_{i-1}(f)\right)_{i}\right),
$$

and the last summand in this expression equals

$$
\left(\pi_{i}-j_{i-1} \circ \pi_{i-1}\right)\left((n+1) \partial^{*}(Z \wedge f)\right),
$$

since $\partial^{*}$ preserves homogeneous degrees. Hence, we see that on $\mathcal{J}^{1}(\mathbb{E})$ we get the equation

$$
\pi_{i} \circ \partial^{*} \circ d_{\mathbb{V}}-j_{i-1} \circ \pi_{i-1} \circ \partial^{*} \circ d_{\mathbb{V}}=\square \circ\left(\pi_{i} \circ p-L_{i-1} \circ \mathcal{J}^{1}\left(\pi_{i-1}\right)\right) .
$$

In fact, this equation is exactly what we were aiming at in the motivation for the whole construction in 4.3. But on the submodule $\tilde{\mathcal{J}}^{1}(\mathbb{E})$, the right hand side of the above formula vanishes identically by Proposition 4.7. Thus, iterated application of this formula shows that on $\tilde{\mathcal{J}}^{1}(\mathbb{E})$ we have

$$
\partial^{*} \circ d_{\mathbb{V}}=\pi_{r+1} \circ \partial^{*} \circ d_{\mathbb{V}}=j_{r} \circ \pi_{r} \circ \partial^{*} \circ d_{\mathbb{V}}=\cdots=j_{1} \circ \pi_{1} \circ \partial^{*} \circ d_{\mathbb{V}} .
$$

But $\pi_{1} \circ \partial^{*} \circ d_{\mathbb{V}}$ maps $(e, Z \otimes f)$ to $\square\left(\pi_{1}(e)\right)$, which vanishes since $\mathbb{E}_{i_{0}}$ is contained in the kernel of the Laplacian, so we have proved $\partial^{*} \circ d_{\mathbb{V}} \circ \mathcal{J}^{1}(L)=$ 0 . All the rest is now an immediate consequence. 
4.10. Definition. Let $(\mathcal{G}, \omega)$ be a (real or complex) parabolic geometry on a manifold $M$. The construction above has given rise to a sequence of strongly invariant operators $D^{\mathbb{V}}$

$$
0 \longrightarrow \Gamma\left(H_{\mathbb{V}}^{0} M\right) \stackrel{D^{\mathbb{V}}}{\longrightarrow} \Gamma\left(H_{\mathbb{V}}^{1} M\right) \stackrel{D^{\mathbb{V}}}{\longrightarrow} \ldots \stackrel{D^{\mathbb{V}}}{\longrightarrow} \Gamma\left(H_{\mathbb{V}}^{\operatorname{dim}(G / P)} M\right) \longrightarrow 0 .
$$

which is called the Bernstein-Gelfand-Gelfand sequence or $B G G-$ sequence determined by the $G$-module $\mathbb{V}$.

All bundles in this sequence correspond to completely reducible representations of $P$, so they all split into direct sums of bundles corresponding to irreducible representations. Let us also remark that the construction applies to both real and complex settings. Next, we will show that in the flat case this sequence is a resolution of the constant sheaf $\mathbb{V}$. Since by Kostant's version of the Bott-Borel-Weil theorem, the bundles occurring in this resolution in the complex case are exactly the same bundles as in the Bernstein-GelfandGelfand resolution, we have obtained curved analogs of this resolution even in the real case.

The main step towards the proof that we often get a resolution is formulated in the next lemma for the general real curved case. For the complex analog see below.

4.11. Lemma. Let $(\mathcal{G}, \omega)$ be a real parabolic geometry on a manifold $M$ and let $s \in \Omega^{n}(M ; V M)$ be a $V M$-valued $n$-form. Then:

(1) There is an element $t \in \Omega^{n-1}(M ; V M)$ such that $s+d_{\mathbb{V}}(t)$ lies in $\operatorname{ker}\left(\partial^{*}\right)$.

(2) If $s$ and $d_{\mathbb{V}}(s)$ both lie in $\operatorname{ker}\left(\partial^{*}\right)$, then $s=L\left(\pi_{H}(s)\right)$.

(3) If $d_{\mathbb{V}}^{2}\left(\operatorname{ker}\left(\partial^{*}\right)\right) \subset \operatorname{ker}\left(\partial^{*}\right)$, then the diagram

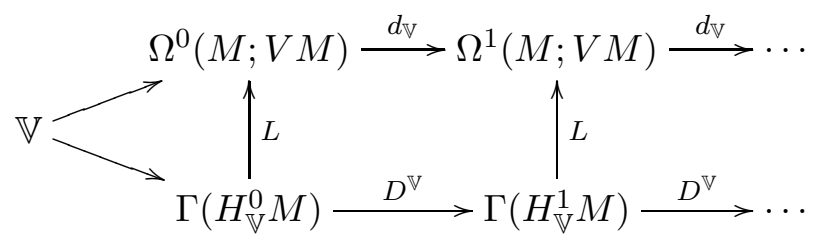

is commutative. In particular, $D^{\mathbb{V}} \circ D^{\mathbb{V}}=0$ whenever $d_{\mathbb{V}} \circ d_{\mathbb{V}}=0$.

Proof. (1) Put $\mathcal{G}_{0}=\mathcal{G} / P_{+}$and choose a global $G_{0}$-equivariant section $\sigma: \mathcal{G}_{0} \rightarrow \mathcal{G}$ as indicated in 2.13. Then we get a smooth map $\tau: \mathcal{G} \rightarrow P_{+}$ characterized by $u=\sigma(p(u)) \cdot \tau(u)$ for all $u \in \mathcal{G}$, and $u \mapsto(p(u), \tau(u))$ is a diffeomorphism $\mathcal{G} \rightarrow \mathcal{G}_{0} \times P_{+}$. Using this, we get an isomorphism (depending on $\sigma)$ between $\Omega^{n}(M ; V M)$ and the space of smooth $G_{0}$-equivariant functions $\mathcal{G}_{0} \rightarrow \Lambda^{n} \mathfrak{p}_{+} \otimes \mathbb{V}$. But $\square^{-1} \circ \partial^{*}$ is a $G_{0}$-homomorphism $\Lambda^{n} \mathfrak{p}_{+} \otimes \mathbb{V} \rightarrow$

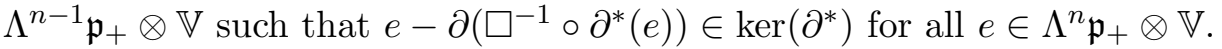

Now, let $\underline{s}: \mathcal{G}_{0} \rightarrow \Lambda^{n} \mathfrak{p}_{+} \otimes \mathbb{V}$ be the $G_{0}$-equivariant map corresponding to the lowest homogeneous component $s_{j}$ of the given $n$-form $s$ such that $\partial^{*}\left(s_{j}\right) \neq 0$. Passing from $-\square^{-1} \circ \partial^{*} \circ \underline{s}$ back to a $P$-equivariant map $t: \mathcal{G} \rightarrow \Lambda^{n-1} \mathfrak{p}_{+} \otimes \mathbb{V}$, we see that the homogeneous components up to degree $j$ of $\partial^{*}\left(s+d_{\mathbb{V}}(t)\right)$ vanish on the image of $\sigma$ and thus on the whole $\mathcal{G}$ by equivariancy. Inductively, we can find an element $t$ with the required properties.

(2) Put $s^{\prime}=\pi_{H}(s)$. By construction of the operators $L$, we know that $L\left(s^{\prime}\right) \in \operatorname{ker}\left(\partial^{*}\right), \pi_{H}\left(L\left(s^{\prime}\right)\right)=s^{\prime}$, and $d_{\mathbb{V}}\left(L\left(s^{\prime}\right)\right) \in \operatorname{ker}\left(\partial^{*}\right)$. Thus, we see that 
$s-L\left(s^{\prime}\right) \in \operatorname{im}\left(\partial^{*}\right)$ and $d_{\mathbb{V}}\left(s-L\left(s^{\prime}\right)\right) \in \operatorname{ker}\left(\partial^{*}\right)$. Let $a_{j}$ be the lowest possibly nonzero homogeneous component of $s-L\left(s^{\prime}\right)$. Then the lowest possibly nonzero component of $d_{\mathbb{V}}\left(s-L\left(s^{\prime}\right)\right)$ is $\partial\left(a_{j}\right)$. Since $\operatorname{ker}\left(\partial^{*}\right)$ is complementary to $\operatorname{im}(\partial)$ we must have $\partial\left(a_{j}\right)=0$. But on the other hand, we know that $a_{j} \in \operatorname{im}\left(\partial^{*}\right)$ which is complementary to $\operatorname{ker}(\partial)$, so we must have $a_{j}=0$.

(3) For $s \in \Gamma\left(H_{\mathbb{V}}^{n} M\right)$, consider the element $d_{\mathbb{V}}(L(s)) \in \Omega^{n+1}(M ; V M)$. By Proposition 4.9, this lies in $\operatorname{ker}\left(\partial^{*}\right)$. Moreover, since $L(s) \in \operatorname{ker}\left(\partial^{*}\right)$, our assumption on $d_{\mathbb{V}}^{2}$ implies that $d_{\mathbb{V}}\left(d_{\mathbb{V}}(L(s))\right) \in \operatorname{ker}\left(\partial^{*}\right)$. Hence from (2) we get $d_{\mathbb{V}}(L(s))=L\left(\pi_{H}\left(d_{\mathbb{V}}(L(s))\right)\right)=L\left(D^{\mathbb{V}}(s)\right)$.

The last claim is obvious.

4.12. Lemma. Let $(\mathcal{G}, \omega)$ be a complex parabolic geometry on a complex manifold $M$. Then the second and third assertion in Lemma 4.11 remain valid with the same assumptions, while the claim 4.11(1) holds true under the additional assumption that the holomorphic bundle $\mathcal{G} \rightarrow \mathcal{G}_{0}$ admits a global holomorphic $G_{0}$-equivariant section. This additional requirement is always fulfilled locally.

Proof. All arguments in the proof of (2) and (3) in 4.11 are on the level of the $P$-modules and so they go equally through for both real and complex settings. The only difference in (1) is the argument which constructs the global section by means of the smooth partition of unity. Once we assume the existence of the global section, the rest is clear again. Now, any point in $M$ has an open neighborhood $U \subset M$ such that both $\mathcal{G}$ and $\mathcal{G}_{0}$ are trivial over $U$. Since $G_{0} \times P_{+}$and $P$ are diffeomorphic, and the map in one direction is obviously holomorphic, they are biholomorphic. Thus, the complex parabolic geometry $\left.\mathcal{G}\right|_{U} \rightarrow U$ admits appropriate global holomorphic $G_{0}$-equivariant section.

4.13. Theorem. Let $(\mathcal{G}, \omega)$ be a real parabolic geometry of the type $(G, P)$ on a manifold $M, \mathbb{V}$ be a $G$-module. If the twisted de Rham sequence

$$
0 \longrightarrow \Omega^{0}(M ; V M) \stackrel{d_{\mathbb{V}}}{\longrightarrow} \Omega^{1}(M ; V M) \stackrel{d_{\mathbb{V}}}{\longrightarrow} \ldots \stackrel{d_{\mathbb{V}}}{\longrightarrow} \Omega^{\operatorname{dim}(G / P)}(M ; V M) \rightarrow 0 .
$$

is a complex, then also the Bernstein-Gelfand-Gelfand sequence

$$
0 \longrightarrow \Gamma\left(H_{\mathbb{V}}^{0} M\right) \stackrel{D^{\mathbb{V}}}{\longrightarrow} \Gamma\left(H_{\mathbb{V}}^{1} M\right) \stackrel{D^{\mathbb{V}}}{\longrightarrow} \ldots \stackrel{D^{\mathbb{V}}}{\longrightarrow} \Gamma\left(H_{\mathbb{V}}^{\operatorname{dim}(G / P)} M\right) \longrightarrow 0
$$

defined in 4.10 is a complex, and they both compute the same cohomology.

The same statement is true for complex parabolic geometries $(\mathcal{G}, \omega)$ under the additional requirement that $\mathcal{G} \rightarrow \mathcal{G}_{0}=\mathcal{G} / P_{+}$admits a global holomorphic $G_{0}$-equivariant section.

Remark. In particular, the complex version of the Theorem may be reformulated as follows: If the twisted de Rham sequence induces a complex on the sheaf level, then the same is true for the Bernstein-Gelfand-Gelfand sequence. In particular, if the twisted de Rham sequence induces a resolution of $\mathbb{V}$, then so does the BGG-sequence.

Now, the original representation theoretical version of the (generalized) BGG-resolution follows immediately by duality. Moreover, let us notice that the global $G_{0}$-equivariant section as required in the Theorem always exists 
over a dense open submanifold in the homogeneous space $G / P$ (the so called big cell).

Proof. As we saw in Lemma 4.11, the BGG-sequence forms a complex whenever the twisted de Rham does. So let us assume, we deal with complexes.

Since $d_{\mathbb{V}}^{2}=0,4.11$ (3) implies that $L$ is a morphism of the corresponding complexes, hence the mapping

$$
\Gamma\left(H_{\mathbb{V}}^{n} M\right) \ni s^{\prime} \mapsto L\left(s^{\prime}\right) \in \Omega^{n}(M ; V M)
$$

induces a morphism between the cohomologies.

Next, suppose that $s \in \Omega^{n}(M ; V M), n \geq 1$ is such that $d_{\mathbb{V}}(s)=0$. Then by 4.11 (1) we find an element $t \in \Omega^{n-1}(M ; V M)$ such that $s+d_{\mathbb{V}}(t) \in$ $\operatorname{ker}\left(\partial^{*}\right)$. But then $d_{\mathbb{V}}\left(s+d_{\mathbb{V}}(t)\right)=0$ so by $4.11(2)$ we know that $s+d_{\mathbb{V}}(t)=$ $L\left(\pi_{H}\left(s+d_{\mathbb{V}}(t)\right)\right)$, and thus the mapping defined above is surjective.

Finally, let us assume that $s^{\prime} \in \Gamma\left(H_{\mathbb{V}}^{n-1} M\right)$ is such that there exists a $t \in \Omega^{n-1}(M ; V M)$ with $d_{\mathbb{V}}(t)=L\left(s^{\prime}\right)$. Then by 4.11(1) we may without loss of generality assume that $t \in \operatorname{ker}\left(\partial^{*}\right)$. But by assumption $d_{\mathbb{V}}(t)=L\left(s^{\prime}\right)$, so this is also contained in $\operatorname{ker}\left(\partial^{*}\right)$, and hence $t=L\left(\pi_{H}(t)\right)$ by $4.11(2)$, and thus $L\left(s^{\prime}\right)=d_{\mathbb{V}}\left(L\left(\pi_{H}(t)\right)\right)$ and applying $\pi_{H}$ on both sides we get $s^{\prime}=D^{\mathbb{V}}\left(\pi_{H}(t)\right)$, and so we get an isomorphism in the cohomology groups.

4.14. Corollary. Let $(\mathcal{G}, \omega)$ be a torsion free real parabolic geometry of type $(G, P)$ on $M$. Then the de Rham cohomology of $M$ with coefficients in $\mathbb{K}=\mathbb{R}$ or $\mathbb{C}$ is computed by the (much smaller) complex

$$
0 \longrightarrow \Gamma\left(H_{\mathbb{K}}^{0} M\right) \stackrel{D^{\mathbb{K}}}{\longrightarrow} \Gamma\left(H_{\mathbb{K}}^{1} M\right) \stackrel{D^{\mathbb{K}}}{\longrightarrow} \ldots \stackrel{D^{\mathbb{K}}}{\longrightarrow} \Gamma\left(H_{\mathbb{K}}^{\operatorname{dim}(G / P)} M\right) \longrightarrow 0 .
$$

Proof. The covariant exterior differential corresponding to the choice of the trivial $P$-module $\mathbb{K}$ coincides with the usual exterior differential $d$. According to Lemma 3.11, the exterior covariant differential coincides with our twisted exterior differential for all torsion-free geometries. Thus the statement follows from 4.13 .

4.15. Corollary. Let $(\mathcal{G}, \omega)$ be a flat real parabolic geometry. Then for any representation $\mathbb{V}$ of $G$ the $B G G$-sequence

$$
0 \longrightarrow \Gamma\left(H_{\mathbb{V}}^{0} M\right) \stackrel{D^{\mathbb{V}}}{\longrightarrow} \Gamma\left(H_{\mathbb{V}}^{1} M\right) \stackrel{D^{\mathbb{V}}}{\longrightarrow} \ldots \stackrel{D^{\mathbb{V}}}{\longrightarrow} \Gamma\left(H_{\mathbb{V}}^{\operatorname{dim}(G / P)} M\right) \longrightarrow 0
$$

is a complex, which computes the twisted de Rham cohomology of $M$ with coefficients in the bundle VM, which is defined as the cohomology of the complex given by the covariant exterior derivative with respect to the linear connection on VM induced by the Cartan connection $\omega$, see 3.11 .

The importance of this corollary lies in the fact that while flat parabolic geometries are locally isomorphic to the homogeneous model $G / P$, they may be very different from $G / P$ from a global point of view. Just keep in mind the broad variety of smooth manifolds admitting a locally conformally flat Riemannian metric. In particular, the bundle $V M$ is not trivial in general, so the twisted de Rham cohomology is a less trivial object than in the homogeneous case. 
On the other hand, we may always consider the obvious flat parabolic

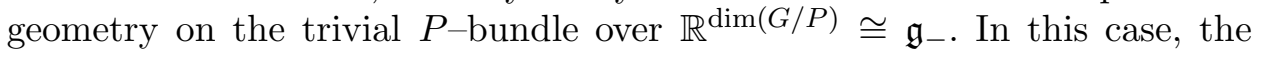
twisted de Rham cohomologies are obviously zero, so Corollary 4.15 provides global resolutions of the constant sheaf $\mathbb{V}$ in this case. Simple instances of such sequences are of basic importance in various areas of mathematics, see for example [14].

4.16. Remark. As we have seen already, the $P$-modules $\mathbb{H}_{\mathbb{V}}^{n}$ are completely reducible and so the natural bundles $H_{\mathbb{V}}^{n} M$ decompose into direct sums of irreducible bundles. Consequently, also the operators $D^{\mathbb{V}}$ split into sums of operators between the irreducible natural bundles. In the case of the homogeneous bundles, the latter operators (and sometimes also their nontrivial compositions) are usually referred to as standard invariant operators. In particular, our construction provides a distinguished curved analog for each of those standard operators.

As we have underlined already in the introduction, no deep representation theoretical results had to be applied in the construction of the BGGsequences and in the proof of Theorem 4.13. On the other hand, the full information of the Kostant's version of Bott-Borel-Weil theorem on the Lie algebra cohomologies is strictly necessary in order to get more explicit information about the individual standard operators and the overall structure of the BGG-sequence in the flat case. Moreover, further non-trivial operators with curvature contributions in their symbols may appear in general.

Let us also remark that the explicit formulae for the standard operators were given in closed form in the terms of the underlying linear connections on $M$ in [10] for all parabolic geometries with irreducible tangent bundles, i.e. for all cases with $|1|$-graded Lie algebra $\mathfrak{g}$. We believe that the technique developed there should be extendible to the general case, too.

4.17. Remark. In the flat case, the twisted de Rham complex can be viewed as a filtered complex with the filtration given by homogeneous degrees. The fact that the lowest homogeneous component of $d_{\mathbb{V}}$ is just $\partial$ implies that the differential on the associated graded complex is exactly $\partial$. Associated to this filtration there is a spectral sequence which obviously converges and computes the twisted de Rham cohomology. Now from the construction of the operators $D^{\mathbb{V}}$ it is obvious that when passing to the appropriate subquotients, they induce the higher differentials in this spectral sequence. Usually, these higher differentials are only well defined on the appropriate subquotients, but due to the fact that we have a (fairly simple) Hodge structure on the associated graded complex, we can get a global definition in our setting.

\section{EXAMPLE}

We shall illustrate the power of our results in the simple case of 5dimensional partially integrable almost $\mathrm{CR}$-structures, cf. Example 2.9. We believe that this simple geometry reflects many of the general features of parabolic geometries and we can still write down the whole BGG-sequences very explicitly at the same time. We hope that based on this example, the 
reader is able to imagine the vast amount of invariant operators which our main theorems produce for all parabolic geometries.

Let $M$ be a smooth manifold of odd dimension $2 n+1$ together with a distinguished rank $n$ complex subbundle $T^{C R} M$ of the tangent bundle $T M$. Then the Lie bracket of vector fields induces a skew-symmetric bundle map $\mathcal{L}^{\mathbb{R}}: T^{C R} M \times T^{C R} M \rightarrow T M / T^{C R} M$, the real Levi-Form. $\left(M, T^{C R} M\right)$ is called a partially integrable almost $C R$-manifold if and only if $\mathcal{L}$ is nondegenerate and totally real, i.e. $\mathcal{L}(J(\xi), J(\eta))=\mathcal{L}(\xi, \eta)$ for all $\xi, \eta \in T^{C R} M$, where $J$ denotes the almost complex structure on $T^{C R} M$. In that case, choosing a local trivialization of $T M / T^{C R} M, \mathcal{L}$ is the imaginary part of a Hermitian form. Here we consider the case where $n=2$, so $M$ has dimension 5 and this Hermitian form is positive definite (for an appropriate choice of the local trivialization).

Typical examples of such manifolds are smooth hypersurfaces in a sixdimensional smooth manifold $N$ endowed with an almost complex structure $\tilde{J}$, which satisfy a non-degeneracy and an integrability condition. In this case, we put $T^{C R} M=T M \cap \tilde{J}(T M)$ and $J=\left.\tilde{J}\right|_{T^{C R} M}$. To understand the non-degeneracy and integrability conditions, it is more convenient to pass to complexified tangent bundles. Since $T^{C R} M$ is a complex bundle, its complexification $T_{\mathbb{C}}^{C R} M$ splits into a direct sum $T_{1,0} M \oplus T_{0,1} M$ of a holomorphic and an antiholomorphic part. Moreover, mapping $\xi, \eta \in \Gamma\left(T_{1,0} M\right)$ to the class of $-i[\xi, \bar{\eta}]$ defines a bundle valued Hermitian form $\mathcal{L}: T_{1,0} M \times T_{1,0} M \rightarrow$ $T_{\mathbb{C}} M / T_{\mathbb{C}}^{C R} M=: Q M$, the Levi form. The partial integrability condition from above is then equivalent to the fact that $[\xi, \eta] \in \Gamma\left(T_{\mathbb{C}}^{C R} M\right)$ for all sections $\xi, \eta$ of $T_{1,0} M$, and the conditions of positive definiteness is equivalent to $\mathcal{L}$ being positive definite in an appropriate local trivialization of $Q M$. (Certainly, these conditions also make sense for abstract almost CR manifolds). A partially integrable almost CR manifold is called integrable or a $C R-$ manifold if and only if the subbundle $T_{1,0} M$ is involutive. In particular, this is the case for hypersurfaces in complex manifolds.

By [7, 4.14], 5-dimensional partially integrable almost CR-manifolds are exactly the normal parabolic geometries corresponding to $G=P S U(3,1)$ and the parabolic subalgebra of $\mathfrak{g}=\mathfrak{s u}(3,1)$ corresponding to the Dynkin diagram $\longleftrightarrow$. Let us also consider $\tilde{G}=S U(3,1)$ and let $P, G_{0} \subset G$, or $\tilde{P}, \tilde{G}_{0} \subset \tilde{G}$ be the corresponding subgroups as in 2.3. Then the semisimple part of $\tilde{G}_{0}$ is $S U(2)$ and the center of $G_{0}$ is $\mathbb{C}$.

In the Dynkin diagram notation, each (complex) irreducible $\tilde{G}$-module $\mathbb{V}$ is given by the choice of three non-negative integers $a, b, c$

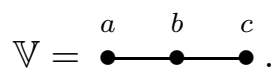

More explicitly, $\stackrel{a}{\bullet} \quad \begin{array}{ll}b \\ \bullet\end{array}$ is the highest weight component in $S^{a} \mathbb{C}^{4} \otimes$ $S^{b}\left(\Lambda^{2} \mathbb{C}^{4}\right) \otimes S^{c}\left(\mathbb{C}^{4 *}\right)$, where $S^{i}$ denotes the $i$-th symmetric power, and so these representations integrate to representation of $G$ if and only if $a-c$ is congruent to $2 b$ modulo four (the center of $\tilde{G}$ consist of \pm 1 and $\pm i$ times the identity).

The irreducible $\tilde{P}$-modules correspond to choices with $b$ non-negative while $a$ and $c$ may be arbitrary integers. Now, $b$ determines the representation of $S U(2)$ while the other two parameters describe the action of the 
center of $\tilde{G}_{0}$. We adopt the convention used in [2], i.e. the parameters give the linear combination of the fundamental weights of $\tilde{\mathfrak{g}}$ which is the highest weight of the dual module to $\mathbb{V}$. In this way, the resulting weights for our modules happen to be the same as those in the dual pictures known from representation theory. For our purposes, however, this has no importance and it is enough to say that the distinguished two subbundles $T_{1,0} M$ and $T_{0,1} M$ in the complexified tangent space and the complexified quotient $Q M=T_{\mathbb{C}} M / T_{\mathbb{C}}^{C R} M$ have duals $T_{1,0}^{*} M, T_{0,1}^{*} M$ (quotients of the complexified cotangent bundle), and $Q^{*} M$, which correspond to the modules

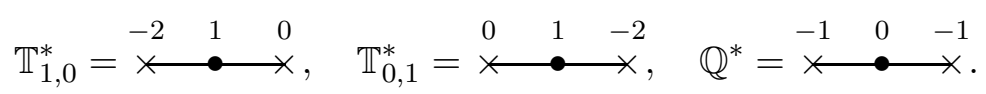

Now, all $\tilde{P}$-modules are tensor products of symmetric powers $S^{b}\left(\mathbb{T}_{1,0}^{*}\right)$ and suitable one-dimensional representations $\mathbb{E}[a, c]$ corresponding to the Dynkin

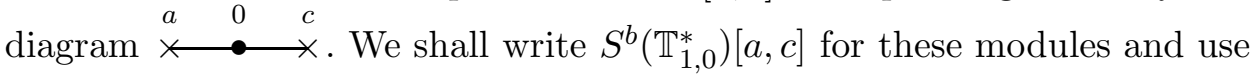
the shorthand $\mathcal{S}_{[a, c]}^{b}$ for the corresponding natural bundles. In particular,

$$
\begin{gathered}
\mathcal{S}_{[a, c]}^{b}=S^{b}\left(\mathbb{T}_{1,0}^{*}\right)[a, c]=\stackrel{a-2 b \quad b}{\longleftarrow} \stackrel{c}{\longleftrightarrow} \\
T_{0,1}^{*}=T_{1,0}^{*}[2,-2]=\mathcal{S}_{[2,-2]}^{1} \\
\mathcal{S}_{[-1,-1]}^{0}=E[-1,-1]=Q^{*} \\
\mathcal{S}_{[-4,0]}^{0}=\Lambda^{2} T_{1,0}^{*} \otimes Q^{*} .
\end{gathered}
$$

Another important bundle is the dual to the kernel of the bilinear Levi form $(\operatorname{ker} \mathcal{L})^{*} \subset T_{1,0}^{*} \otimes T_{0,1}^{*}$ which corresponds to $\mathcal{S}_{[2,-2]}^{2}$.

All natural bundles $\mathcal{S}_{[a, c]}^{b}$ exist on manifolds $M$ with the so called $S U(3,1)$ structures, i.e. we have to choose coverings of the Cartan $P$-bundle $\mathcal{G}$ to the structure group $\tilde{P}$. This is clearly equivalent to the choice of a fixed line bundle $E[1,0]$ such that its fourth tensor power is $\Lambda^{2} T_{1,0} M \otimes Q M$. This is an analogous situation to natural bundles and natural operators in conformal Riemannian geometry which often depend on the choice of a spin structure.

Using the explicit description of the cohomology from Kostant's BottBorel-Weil theorem we obtain explicitly all natural bundles appearing in our main theorems. The computations are done fairly simply in terms of the Dynkin diagram notation, see [2] for the details. Furthermore, using elementary finite dimensional representation theory one easily shows that there are no homomorphisms between the semi-holonomic jet modules corresponding to the items in the neighboring columns of the BGG-sequences, except those which are indicated in Figure 1. Let us also notice that the orders of the operators are easily read off the homogeneities of the bundles with respect to the action of the grading element in $G_{0}$ and the homogeneity of $\mathcal{S}_{[a, c]}^{b}$ is $a+c-b$. Thus we can summarize:

5.1. Theorem. For each $S U(3,1)-$ module $\mathbb{V}=\stackrel{a}{\bullet} \quad \stackrel{b}{\bullet} \quad \stackrel{c}{\bullet}$, the $B G G$-sequence of invariant differential operators shown on Figure 1 exists on all 5-dimensional partially integrable almost $C R$-manifolds $M$ with a chosen $S U(3,1)$-structure. The orders of the operators are indicated by the labels over the arrows. Moreover, the sequence exists on all partially integrable $C R$-manifolds if 


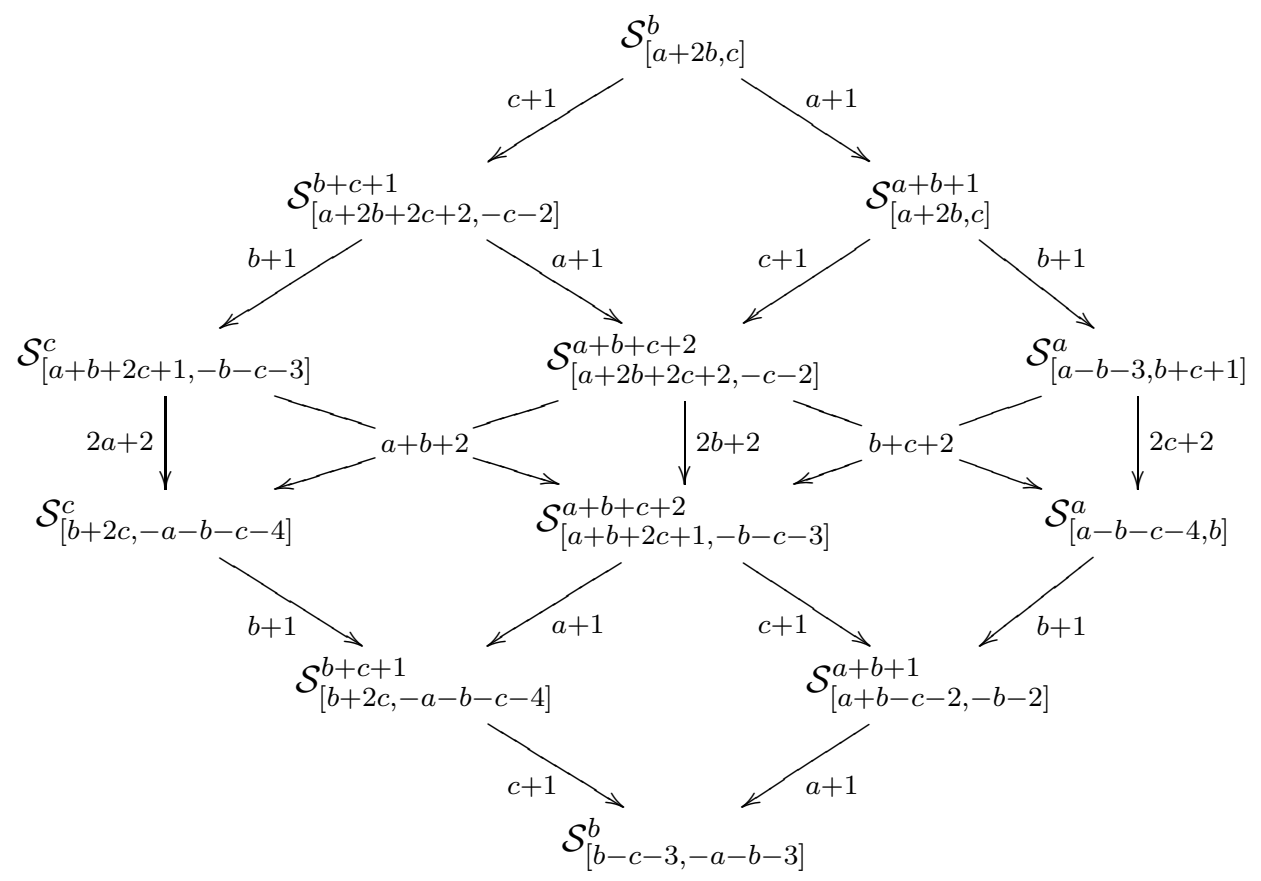

Figure 1. Bernstein-Gelfand-Gelfand sequences on partially integrable 5-dimensional almost CR structures

$a-2 b+c \equiv 0 \bmod 4$, and then all bundles in question can be constructed from $T_{1,0}^{*} M$ and $Q^{*} M$. If $M$ is flat, then the $B G G$-sequence is a complex which computes the twisted de Rham cohomology of $M$ with coefficients in the bundle $V M$ corresponding to $\mathbb{V}$.

As a corollary, we immediately obtain

5.2. Theorem. For all (integrable) 5-dimensional CR-manifolds, there is the resolution of the sheaf of constant complex functions

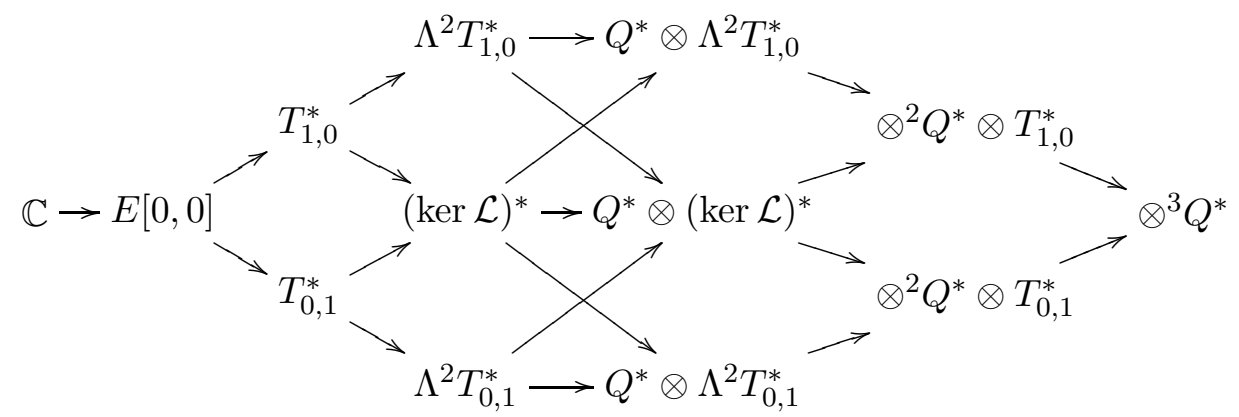

which computes the de Rham cohomology with complex coefficients. The orders of the operators in the column in the middle of the diagram are two, while all the other ones are of first order.

This complex is a special instance of the so called Rumin complex on contact geometries, [28], see also [18] for a refined version for the CR-structures. In the homogeneous case, this complex was also mentioned in [2]. Similar 
questions were also studied by Lychagin earlier, see e.g. 223 and the references therein. Notice that the dimensions of the individual columns are 1,4 , 5, 5, 4, 1 (opposed to dimensions $1,5,10,10,5,1$ in the de Rham complex).

\section{Appendix A. Infinite Jets And Verma modules}

The aim of this appendix is to provide differential geometers with basic information on the links between jets and Verma modules, and in particular to prove the correspondence between invariant differential operators and homomorphisms of generalized Verma modules used in 2.6.

A.1. We have seen in 2.6 that invariant operators $\Gamma(E) \rightarrow \Gamma(F)$ between homogeneous vector bundles over $G / P$ are in bijective correspondence with $P$-homomorphisms $J^{\infty}(E)_{o} \rightarrow F_{o}$, which factorize over some $J^{r}(E)_{o}$.

First note that sections of $E$ can be identified with smooth functions $G \rightarrow$ $\mathbb{E}$, which are $P$-equivariant. Since this identification is purely algebraic, it gives an identification of infinite jets at $o$ of sections of $E$ with $P$-equivariant infinite jets of smooth functions $G \rightarrow \mathbb{E}$ at $e \in G$. Now it is easy to verify that in the picture of smooth equivariant functions, the action of $G$ is given by $(g \cdot s)\left(g^{\prime}\right)=s\left(g^{-1} g^{\prime}\right)$. The corresponding infinitesimal action of $\mathfrak{g}$ is given by $(X \cdot s)(g)=-\left(R_{X} \cdot s\right)(g)$, where $R_{X}$ denotes the right-invariant vector field on $G$ generated by $X \in \mathfrak{g}=T_{e} G$. For $X \in \mathfrak{p}$, the infinitesimal version of equivariancy of $s$ implies that $(X \cdot s)(g)=X \cdot(s(g))$, but for general $X$ the value $(X \cdot s)(g)$ depends on the one-jet of $s$ at $g$. Thus we do not get an induced action of $\mathfrak{g}$ on finite jets, but for infinite jets we get a well defined action of $\mathfrak{g}$. Since this action is clearly compatible with the action of $P$, it makes $J^{\infty}(E)_{o}$ into a $(\mathfrak{g}, P)$-module.

On the other hand, mapping each $X \in \mathfrak{g}$ to the left invariant vector field $L_{X}$ generated by $X$ induces an isomorphism between the universal enveloping algebra $\mathcal{U}(\mathfrak{g})$ and the algebra of left invariant differential operators on $G$. Now we get a bilinear map $J^{\infty}(E)_{o} \times\left(\mathcal{U}(\mathfrak{g}) \otimes \mathbb{E}^{*}\right) \rightarrow \mathbb{K}$ by mapping $\left(j^{\infty} s(e), D \otimes \lambda\right)$ to $\lambda(D(s)(e))$, where $D$ is a left invariant differential operator and $\lambda$ is an element of the dual representation $\mathbb{E}^{*}$ to $\mathbb{E}$, and as above we view $s$ as an equivariant function on $G$. By equivariancy of $s$ this factors to a bilinear map $J^{\infty}(E)_{o} \times\left(\mathcal{U}(\mathfrak{g}) \otimes_{\mathcal{U}(\mathfrak{p})} \mathbb{E}^{*}\right) \rightarrow \mathbb{K}$ because elements of $\mathcal{U}(\mathfrak{p})$ act algebraically and this can be expressed as an action on $\lambda$.

We claim that the above pairing is compatible with the actions of both $\mathfrak{g}$ and $P$. For the action of $\mathfrak{g}$, let us take a typical element $X_{1} \otimes \cdots \otimes$ $X_{n} \otimes \lambda \in \mathcal{U}(\mathfrak{g}) \otimes_{\mathcal{U}(\mathfrak{p})} \mathbb{E}^{*}$ and $X \in \mathfrak{g}$. From above, we know that $X \cdot j^{\infty} s(e)=$ $-j^{\infty}\left(R_{X} \cdot s\right)(e)$. Pairing this with $X_{1} \otimes \cdots \otimes X_{n} \otimes \lambda$, we get $-\lambda\left(\left(L_{X_{1}} \ldots L_{X_{n}} \cdot R_{X} \cdot s\right)(e)\right)$. Since left invariant vector fields commute with right invariant ones, this equals $-\lambda\left(\left(R_{X} \cdot L_{X_{1}} \ldots L_{X_{n}} \cdot s\right)(e)\right)$. But this depends only on $R_{X}(e)$, so we may as well replace $R_{X}$ by $L_{X}$, so this coincides with $X \otimes X_{1} \otimes \cdots \otimes X_{n} \otimes \lambda$ evaluated on $j^{\infty} s(e)$.

The action of $b \in P$ on $\mathcal{U}(\mathfrak{g}) \otimes_{\mathcal{U}(\mathfrak{p})} \mathbb{E}^{*}$ is induced by mapping $D \otimes \lambda$ to $b \cdot D \otimes b \cdot \lambda$, where $(b \cdot D)(s)=D\left(s \circ r^{b^{-1}}\right) \circ r^{b}$ and $r^{b}$ denotes the right multiplication by $b$. This obviously maps the anihilator of the space of $P$ equivariant functions to itself and thus descends to an action on $\mathcal{U}(\mathfrak{g}) \otimes_{\mathcal{U}}(\mathfrak{p})$ $\mathbb{E}^{*}$. If $s$ is equivariant, then $\left(s \circ r^{b^{-1}}\right)(g)=b \cdot(s(g))$, and thus $(b \cdot D)(s)(g)=$ 
$b \cdot(D(s)(g b))$. But this implies that pairing $j^{\infty} s(e)$ with $b \cdot D \otimes b \cdot \lambda$ we get $(b \cdot \lambda)((b \cdot D)(s)(e))=\lambda(D(s)(b))$. On the other hand, the action of $b$ on $J^{\infty}(E)_{o}$ is given by $b \cdot j^{\infty} s(e)=j^{\infty}\left(s \circ \ell_{b^{-1}}\right)(e)$, where $\ell_{b}$ denotes the left multiplication by $b$. Thus pairing $b^{-1} \cdot j^{\infty} s(e)$ with $D \otimes \lambda$ we get $\lambda\left(D\left(s \circ \ell_{b}\right)(e)\right)$, which by left invariance of $D$ coincides with $\lambda(D(s)(b))$.

Now for any $k \in \mathbb{N}$, we have the natural projection $J^{\infty}(E)_{o} \rightarrow J^{k}(E)_{o}$. On the other hand, the universal enveloping algebra $\mathcal{U}(\mathfrak{g})$ has a natural (infinite) filtration $\mathbb{K}=\mathcal{U}^{0}(\mathfrak{g}) \subset \mathcal{U}^{1}(\mathfrak{g}) \subset \ldots$ such that $\mathcal{U}(\mathfrak{g})=\cup_{i \in \mathbb{N}} \mathcal{U}^{i}(\mathfrak{g})$. In the picture of left invariant differential operators on $G$, this is just the filtration by the order of operators. This filtration clearly induces a filtration $\mathcal{F}^{i}$ on $\mathcal{U}(\mathfrak{g}) \otimes \mathcal{U}(\mathfrak{p}) \mathbb{E}^{*}$, and each filtration component is a $P$-submodule (but not a $\mathfrak{g}$-submodule). The pairing of an element of $\mathcal{F}^{k}$ with an element $j^{\infty}(s)(e) \in J^{\infty}(E)_{o}$ clearly depends only on $j^{k} s(e)$, so we get an induced paring between $\mathcal{F}^{k}$ and $J^{k}(E)_{o}$, and this induced pairing is obviously nondegenerate and still compatible with the $P$-actions, so since both sides are finite dimensional, they are dual $P$-modules.

Let us remark at this point that it is also possible to put locally convex topologies on the spaces in question, such that they become topologically dual $(\mathfrak{g}, P)$-modules. Namely, one has to view $J^{\infty}(E)_{o}$ as the limit of the system $\cdots \rightarrow J^{k}(E)_{o} \rightarrow J^{k-1}(E)_{o} \rightarrow \ldots$, while $\mathcal{U}(\mathfrak{g}) \otimes_{\mathcal{U}(\mathfrak{p})} \mathbb{E}^{*}$ has to be topologized as a direct sum of finite dimensional spaces.

A.2. Let $\mathbb{E}$ and $\mathbb{F}$ be $P$-representations, $E$ and $F$ the corresponding bundles and $\varphi: J^{k}(E)_{o} \rightarrow F_{o}=\mathbb{F}$ a $P$-homomorphism. By the duality shown above, we can view the dual map $\varphi^{*}$ as a $P$-homomorphism $\mathbb{F}^{*} \rightarrow \mathcal{F}^{k} \subset$ $\mathcal{U}(\mathfrak{g}) \otimes_{\mathcal{U}(\mathfrak{p})} \mathbb{E}^{*}$. Conversely, if we have a $P$-homomorphism $\mathbb{F}^{*} \rightarrow \mathcal{U}(\mathfrak{g}) \otimes_{\mathcal{U}(\mathfrak{p})} \mathbb{E}^{*}$ then this has values in some $\mathcal{F}^{i}$ since $\mathbb{F}^{*}$ is finite dimensional, so dualizing it corresponds to a $P$-homomorphism $J^{i}(E)_{o} \rightarrow F_{o}$. Consequently, we see that the space of invariant operators $\Gamma(E) \rightarrow \Gamma(F)$ is isomorphic to $\operatorname{Hom}_{P}\left(\mathbb{F}^{*}, \mathcal{U}(\mathfrak{g}) \otimes_{\mathcal{U}(\mathfrak{p})} \mathbb{E}^{*}\right)$.

By Frobenius reciprocity the latter space is isomorphic to

$$
\operatorname{Hom}_{(\mathfrak{g}, P)}\left(\mathcal{U}(\mathfrak{g}) \otimes_{\mathcal{U}(\mathfrak{p})} \mathbb{F}^{*}, \mathcal{U}(\mathfrak{g}) \otimes_{\mathcal{U}(\mathfrak{p})} \mathbb{E}^{*}\right) .
$$

This isomorphism is quite simple to prove: If $\varphi: \mathbb{F}^{*} \rightarrow \mathcal{U}(\mathfrak{g}) \otimes_{\mathcal{U}(\mathfrak{p})} \mathbb{E}^{*}$ is a $P$-homomorphism, then $\tilde{\Phi}(A \otimes \lambda)=A \cdot \varphi(\lambda)$ defines a $(\mathfrak{g}, P)$-homomorphism $\mathcal{U}(\mathfrak{g}) \otimes \mathbb{F}^{*} \rightarrow \mathcal{U}(\mathfrak{g}) \otimes_{\mathcal{U}(\mathfrak{p})} \mathbb{E}^{*}$, and since $\varphi$ is a $P$-homomorphism, this factors to a $(\mathfrak{g}, P)$-homomorphism $\Phi$ between the required spaces. Conversely, we put $\varphi(\lambda)=\Phi(1 \otimes \lambda)$ and this clearly is a $P$-homomorphism if $\Phi$ is a $(\mathfrak{g}, P)$ homomorphism.

\section{Appendix B. Adjointness of $\partial$ And $\partial^{*}$}

B.1. As promised in the beginning of Section 1 , we show that the operators $\partial$ and $\partial^{*}$ are adjoint operators with respect to a certain inner product on $C^{n}\left(\mathfrak{g}_{-}, \mathbb{V}\right)$. To construct this inner product, we have to distinguish between the real and the complex case. Let us start with the case where $\mathfrak{g}$ and $\mathbb{V}$ are complex. Since the grading element $E \in \mathfrak{g}_{0}$ is semisimple, we can find a Cartan subalgebra $\mathfrak{h} \subset \mathfrak{g}$ which contains $E$. Then each root space for this Cartan subalgebra is contained in some $\mathfrak{g}_{i}$. Let $\mathfrak{u}$ be a compact real form of $\mathfrak{g}$ with a Cartan subalgebra $\mathfrak{h}_{0}$ contained in $\mathfrak{h}$, and let $\sigma$ be the 
complex conjugation with respect to this real form. By definition of $E$, the $\operatorname{map} \operatorname{ad}(E) \circ \operatorname{ad}(E)$ acts on $\mathfrak{g}_{i}$ by multiplication by $i^{2}$, so for the Killing form we have $B(E, E)>0$. Consequently, we must have $\sigma(E)=-E$, and thus $\sigma\left(\mathfrak{g}_{i}\right)=\mathfrak{g}_{-i}$ for all $i=-k, \ldots, k$. Now one immediately verifies directly that $B^{*}(X, Y):=-B(X, \sigma(Y))$ is a positive definite Hermitian inner product on $\mathfrak{g}$, such that the decomposition $\mathfrak{g}=\mathfrak{g}_{-k} \oplus \cdots \oplus \mathfrak{g}_{k}$ is an orthogonal direct sum. In particular, this induces a Hermitian inner product on $\mathfrak{g}_{-}$.

Next, since $\mathfrak{u}$ is a compact real form, there is a positive definite Hermitian inner product $\langle$,$\rangle on \mathbb{V}$ such that the elements of $\mathfrak{u}$ act as skew-Hermitian operators. But this immediately implies that for each $X \in \mathfrak{g}$ and $v_{1}, v_{2} \in \mathbb{V}$, we have $\left\langle X \cdot v_{1}, v_{2}\right\rangle=-\left\langle v_{1}, \sigma(X) \cdot v_{2}\right\rangle$. Together with the inner product on

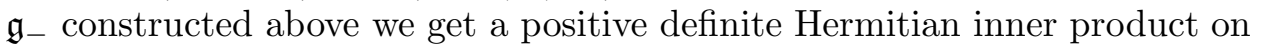
$C^{n}\left(\mathfrak{g}_{-}, \mathbb{V}\right)$ for each $n$.

In the real case, the situation is slightly more complicated. In this case we have to construct appropriate involutions $\sigma$ on the individual simple factors separately, and we have to distinguish between the case where the complexification of a simple factor is again simple and the case where it is not. Note that the simple factors of a $|k|$-graded Lie algebra are themselves $|\ell|$-graded for some $\ell \leq k$ and that the grading element of $\mathfrak{g}$ is just the sum of the grading elements of the simple factors.

If we have a real simple algebra $\mathfrak{g}$ whose complexification is not simple, then it is well known that $\mathfrak{g}$ is actually the underlying real Lie algebra of a complex simple Lie algebra. In this case, we can proceed as above to get a compact real form $\mathfrak{u} \subset \mathfrak{g}$ and the corresponding involution $\sigma$.

In the case where both $\mathfrak{g}$ and its complexification $\mathfrak{g}^{\mathbb{C}}$ are simple, we choose a Cartan subalgebra $\mathfrak{h} \subset \mathfrak{g}^{\mathbb{C}}$ which contains the element $E \in \mathfrak{g}$. By 29, Exposè 11, Théorème 3] there is a compact real form $\mathfrak{u} \subset \mathfrak{g}^{\mathbb{C}}$ with Cartan subalgebra $\mathfrak{h}_{0} \subset \mathfrak{h}$ such that the complex conjugation $\sigma$ with respect to $\mathfrak{u}$ commutes with the complex conjugation with respect to $\mathfrak{g}$, and thus $\sigma(\mathfrak{g})=$ $\mathfrak{g}$.

The involutions on the simple factor together define an involution of $\mathfrak{g}$ and as above one uses the Killing form on $\mathfrak{g}$ and $\sigma$ to get a positive definite inner product on $\mathfrak{g}$ and on $\mathfrak{g}_{-}$. If the representation $\mathbb{V}$ is not already complex, then we can pass to its complexification to get a Hermitian inner product such that $\left\langle X \cdot v_{1}, v_{2}\right\rangle=-\left\langle v_{1}, \sigma(X) \cdot v_{2}\right\rangle$ as above, an in both cases the real part of this Hermitian product gives a positive definite inner product on $\mathbb{V}$ which we use together with the inner product on $\mathfrak{g}_{-}$to get a positive definite inner product on $C^{n}\left(\mathfrak{g}_{-}, \mathbb{V}\right)$.

B.2. Proposition. The differential $\partial: C^{n}\left(\mathfrak{g}_{-1}, \mathbb{V}\right) \rightarrow C^{n+1}\left(\mathfrak{g}_{-1}, \mathbb{V}\right)$ and the codifferential $\partial^{*}: C^{n+1}\left(\mathfrak{g}_{-1}, \mathbb{V}\right) \rightarrow C^{n}\left(\mathfrak{g}_{-1}, \mathbb{V}\right)$ are adjoint operators with respect to the inner products constructed in B.1 above.

Proof. The point about this is that in each case the inner product of $f_{1}, f_{2} \in$ $C^{n}\left(\mathfrak{g}_{-}, \mathbb{V}\right)$ can be computed as $\mathcal{F}\left(f_{2}\right)\left(f_{1}\right)$, where $\mathcal{F}$ is a linear (over the reals) isomorphism $C^{n}\left(\mathfrak{g}_{-}, \mathbb{V}\right) \rightarrow C^{n}\left(\mathfrak{p}_{+}, \mathbb{V}^{*}\right)$. The map $\mathcal{F}$ is defined by $\mathcal{F}(f)\left(Z_{1}, \ldots, Z_{n}\right)(v):=\left\langle f\left(\sigma\left(Z_{1}\right), \ldots, \sigma\left(Z_{n}\right)\right), v\right\rangle$ for $Z_{i} \in \mathfrak{p}_{+}$and $v \in \mathbb{V}$, where $\sigma$ is the involution constructed in B.1 and the inner product is in $\mathbb{V}$. But then the compatibility of the inner product on $\mathbb{V}$ with the action of $\mathfrak{g}$ 
implies that $\mathcal{F}(\partial(f))=\partial(\mathcal{F}(f))$. Thus we can compute:

$$
\begin{aligned}
\left\langle\partial^{*}\left(f_{1}\right), f_{2}\right\rangle=\mathcal{F}\left(f_{2}\right)\left(\partial^{*}\left(f_{1}\right)\right)=\partial\left(\mathcal{F}\left(f_{2}\right)\right)\left(f_{1}\right) & \\
& =\mathcal{F}\left(\partial\left(f_{2}\right)\right)\left(f_{1}\right)=\left\langle f_{1}, \partial\left(f_{2}\right)\right\rangle
\end{aligned}
$$

\section{REFERENCES}

[1] R.J. Baston, Almost Hermitian symmetric manifolds, I: Local twistor theory; II: Differential invariants, Duke Math. J., 63 (1991), 81-111, 113-138

[2] R. J. Baston, M. G. Eastwood, "The Penrose Transform" Its Interaction with Representation Theory, Oxford Science Publications, Clarendon Press, 1989

[3] I.N. Bernstein, I.M. Gelfand, S.I. Gelfand, Differential operators on the base affine space and a study of $\mathfrak{g}$-modules, in "Lie Groups and their Representations" (ed. I.M. Gelfand) Adam Hilger 1975, 21-64

[4] B.D. Boe, D.H. Collingwood, A comparison theory for the structure of induced representations I., J. of Algebra 94 (1985) 511-545

[5] B.D. Boe, D.H. Collingwood, A comparison theory for the structure of induced representations II., Math. Z. 190 (1985) 1-11

[6] A. Cap, Translation of natural operators on manifolds with AHS-structures, Archivum Math. (Brno) 32, 4 (1996), 249-266, electronically available at www.emis.de

[7] A. Čap, H. Schichl, Parabolic Geometries and Canonical Cartan Connections, Preprint ESI 450, electronically available at www.esi.ac.at

[8] A. Čap, J. Slovák, V. Souček, Invariant operators on manifolds with almost Hermitian symmetric structures, I. Invariant differentiation, Acta Math. Univ. Commenianae, 66 (1997), 33-69, electronically available at www.emis.de

[9] A. Čap, J. Slovák, V. Souček, Invariant operators on manifolds with almost Hermitian symmetric structures, II. Normal Cartan connections, Acta Math. Univ. Commenianae, 66 (1997), 203-220, electronically available at www.emis.de

[10] A. Čap, J. Slovák, V. Souček, Invariant operators on manifolds with almost Hermitian symmetric structures, III. Standard Operators, ESI Preprint 613, to appear in Jour. Diff. Geom. Appl., electronically available at www.esi.ac.at

[11] E. Cartan, Les espaces à connexion conforme, Ann. Soc. Pol. Math., 2 (1923), 171202

[12] M.G. Eastwood, Notes on conformal geometry, in Proc. of the 15th Winter School Geometry and Physics, Srnií 1995, Supp. ai Rend. Circ. Mat. Palermo, vol. 43, 1996, $57-76$

[13] M.G. Eastwood, Variations on the de Rham complex, to appear in Notices of AMS

[14] M.G. Eastwood, A complex from linear elasticity, to appear in Proc. of the 19th Winter School Geometry and Physics, Srní 1999, Supp. ai Rend. Circ. Mat. Palermo

[15] M.G. Eastwood; J.W. Rice, Conformally invariant differential operators on Minkowski space and their curved analogues, Commun. Math. Phys. 109 (1987), $207-228$

[16] M.G. Eastwood, J. Slovák, Semi-holonomic Verma modules, J. of Algebra, 197 (1997), 424-448

[17] C. Fefferman, Parabolic invariant theory in complex analysis, Adv. in Math. 31 (1979), 131-262

[18] P. Garfield, J.M. Lee, The Rumin complex on CR manifolds, to appear

[19] A. R. Gover, Conformally invariant operators of standard type, Quart. J. Math. 40 (1989), 197-208

[20] C.R. Graham, Conformally invariant powers of the Laplacian, II: Nonexistence, J. London Math. Soc., 46 (1992), 566-576

[21] C.R. Graham, R. Jenne, L.J. Mason, G.A. Sparling, Conformally invariant powers of the Laplacian, I: Existence, J. London Math. Soc., 46 (1992), 557-565

[22] J. E. Humphreys, "Introduction to Lie Algebras and Representation Theory" Grad. Texts in Math. Vol. 9, Springer 1972 
[23] V. V. Lychagin, Homogeneous structures on manifolds: differential geometry from the point of view of differential equations, Matematicheskie zametki (in russian), 51, 4 (1992), 54-68

[24] I. Kolář, P.W. Michor, J. Slovák, "Natural Operations in Differential Geometry", Springer 1993

[25] B. Kostant, Lie algebra cohomology and the generalized Borel-Weil theorem, Ann. Math. 74 No. 2 (1961), 329-387

[26] J. Lepowsky, A generalization of the Bernstein-Gelfand-Gelfand resolution, J. of Algebra 49 (1977) 496-511

[27] T. Morimoto, Geometric structures on filtered manifolds, Hokkaido Math. J. 22, (1993), 263-347

[28] M. Rumin, Formes differentielles sur les variétes de contact, J. Differential Geom. 39 (1994), 281-330

[29] Séminaire "Sophus Lie", 1e année: 1954/55, Théorie des algèbres de Lie, Topologie des groupes de Lie, Ecole Norm. Sup.

[30] G. Schmalz; J. Slovák, The Geometry of Hyperbolic and Elliptic CR-manifolds of codimension two, Preprint MPI MIS 3/99, electronically available at www.mis.mpg.de

[31] R.W. Sharpe, "Differential Geometry", Graduate Texts in Mathematics 166, Springer-Verlag 1997

[32] J. Slovák, On the geometry of almost Hermitian symmetric structures, in "Proceedings of the Conference Differential Geometry and Applications, 1995, Brno", Masaryk University, Brno (1996), 191-206, electronically available at www.emis.de

[33] J. Slovák, Parabolic geometries, Research Lecture Notes, Part of DrSc. Dissertation, Preprint IGA 11/97, electronically available at www.maths.adelaide.edu.au, 70pp

[34] N. Tanaka, On the equivalence problem associated with simple graded Lie algebras, Hokkaido Math. J., 8 (1979), 23-84

[35] K. Yamaguchi, Differential systems associated with simple graded Lie algebras, Advanced Studies in Pure Mathematics 22 (1993), 413-494

A.C.: Institut für Mathematik, Universität Wien, Strudlhofgasse 4, A-1090 Wien, Austria and International Erwin Schrödinger Institute for Mathematical Physics, Boltzmanngasse 9, A-1090 Wien, Austria

J.S.: Department of Algebra and Geometry, Masaryk University in Brno, JanÁČKovo nám. 2A, 66295 Brno, Czech Republic

V.S.: Mathematical Institute, Charles University, Sokolovská 83, Praha, Czech Republic

E-mail address: Andreas.Cap@esi.ac.at, slovak@math.muni.cz, soucek@karlin.mff.cuni.cz 